Fall 2017

\title{
DFT Study on the Li Mobility in Li-lon-Based Solid-State Electrolytes
}

Shafiqul Islam

Missouri State University, MdShafiqul055@live.missouristate.edu

As with any intellectual project, the content and views expressed in this thesis may be considered objectionable by some readers. However, this student-scholar's work has been judged to have academic value by the student's thesis committee members trained in the discipline. The content and views expressed in this thesis are those of the student-scholar and are not endorsed by Missouri State University, its Graduate College, or its employees.

Follow this and additional works at: https://bearworks.missouristate.edu/theses

Part of the Materials Science and Engineering Commons

\section{Recommended Citation}

Islam, Shafiqul, "DFT Study on the Li Mobility in Li-lon-Based Solid-State Electrolytes" (2017). MSU

Graduate Theses. 3217.

https://bearworks.missouristate.edu/theses/3217

This article or document was made available through BearWorks, the institutional repository of Missouri State University. The work contained in it may be protected by copyright and require permission of the copyright holder for reuse or redistribution.

For more information, please contact BearWorks@library.missouristate.edu. 


\title{
DFT STUDY ON THE LI MOBILITY IN LI-ION-BASED SOLID-STATE ELECTROLYTES
}

\author{
A Master Thesis \\ Presented to \\ The Graduate College of \\ Missouri State University
}

\author{
In Partial Fulfillment \\ Of the Requirements for the Degree \\ Master of Science, Materials Science
}

\section{By}

Md Shafiqul Islam

December 2017 
Copyright 2017 by Md Shafiqul Islam 


\title{
DFT STUDY ON THE LI MOBILITY IN LI-ION-BASED SOLID-STATE
}

\section{ELECTROLYTES}

Physics, Astronomy, and Materials Science

Missouri State University, December 2017

Master of Science

Md Shafiqul Islam

\begin{abstract}
I have investigated the diffusion mechanisms of Li-ion in amorphous lithium phosphite $\left(\mathrm{LiPO}_{3}\right)$ with addition of sulphur. By applying the nudge elastic band (NEB) method in crystal $\mathrm{LiPO}_{3}$ and $\mathrm{Li}_{3} \mathrm{PO}_{4}$, I confirmed the easing of diffusion pathways for $\mathrm{Li}$ ion in $\mathrm{LiPO}_{3}$ which is consistent with the previous theoretical finding[1]. From the diffusion study in $0.5 \mathrm{Li}_{2} \mathrm{O}-0.5 \mathrm{P}_{2} \mathrm{O}_{5}$ and $0.4 \mathrm{Li}_{2} \mathrm{SO}_{4}-0.6\left(\mathrm{Li}_{2} \mathrm{O}-\mathrm{P}_{2} \mathrm{O}_{5}\right)$ melts above $3000 \mathrm{~K}$ performed with ab-initio molecular dynamics (AIMD), produces identical outcome as experimental at lower temperatures. I demonstrated the benefit of addition of $\mathrm{S}$ in increasing $\mathrm{Li}^{+}$mobility. I also found that the $\mathrm{Li}_{2} \mathrm{SO}_{4}$ addition into the glass results in a characteristic shift in Li-ion vibration to a lower vibrational frequency. In addition, the presence of oxygen surrounding the diffusion pathways appears to be essential in assisting the $\mathrm{Li}^{+}$mobility in both crystalline and amorphous structures. The activation energy barrier shows similar pattern as reported in experimental analysis with $\mathrm{LiPO}_{3}[2]$.
\end{abstract}

KEYWORDS: diffusion, density functional theory, nudge elastic band, ab-initio molecular dynamics, Li-ion battery, solid state electrolyte

This abstract is approved as to form and content

Ridwan Sakidja, $\mathrm{PhD}$

Chairperson, Advisory Committee

Missouri State University 


\title{
DFT STUDY ON THE LI MOBILITY IN LI-ION-BASED SOLID-STATE ELECTROLYTES
}

\author{
By \\ Md Shafiqul Islam \\ A Master Thesis \\ Submitted to the Graduate College \\ Of Missouri State University \\ In Partial Fulfillment of the Requirements \\ For the Degree of Master of Science, Materials Science
}

December 2017

Approved:

Ridwan Sakidja, $\mathrm{PhD}$

Saibal Mitra, $\mathrm{PhD}$

Robert A. Mayanovic, PhD

Julie Masterson, PhD: Dean, Graduate College

In the interest of academic freedom and the principle of free speech, approval of this thesis indicates the format is acceptable and meets the academic criteria for the discipline as determined by the faculty that constitute the thesis committee. The content and views expressed in this thesis are those of the studentscholar and are not endorsed by Missouri State University, its Graduate College, or its employees. 


\section{ACKNOWLEDGEMENTS}

I would like to thank you, my advisor, Dr. Ridwan Sakidja, for all his help in my research within two years of stay in MSU. I also want to thank my Mother, Father, and sister, for, without their support and cooperation, my thesis will not be in the shape it is

today. Last, I want to thank the Physics, Astronomy, and Material Science Department in MSU for providing a friendly work environment for me to complete my study. I also want to thank my college Mr. Paul Simanjuntak for his support towards my thesis completion. 


\section{TABLE OF CONTENTS}

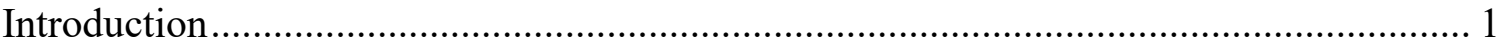

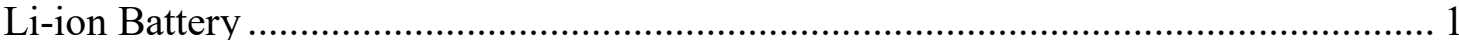

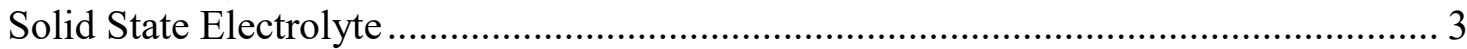

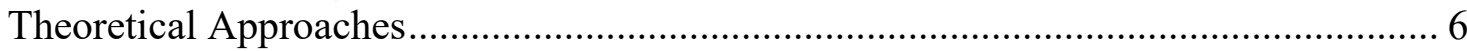

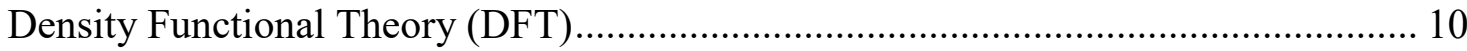

Minimum Energy Path (MEP) …………………………………………………..... 19

Nudge Elastic Band (NEB) ................................................................................... 20

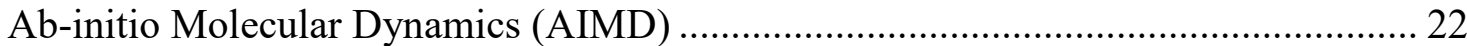

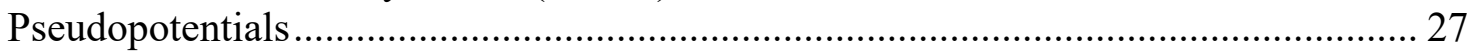

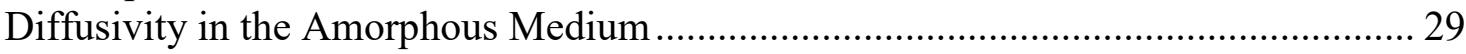

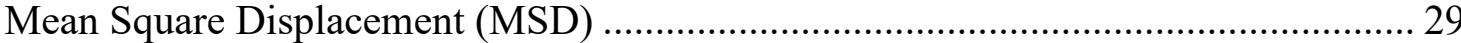

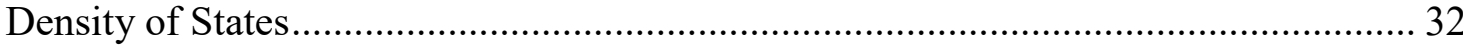

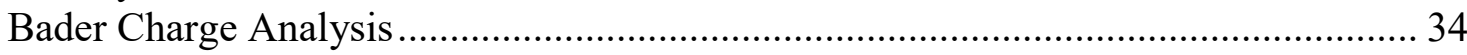

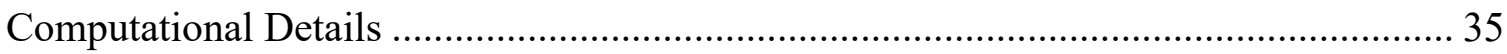

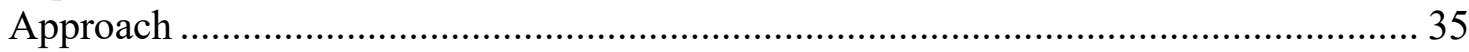

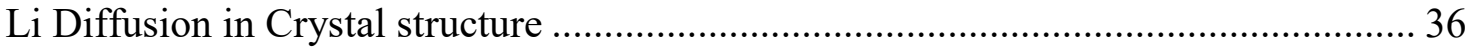

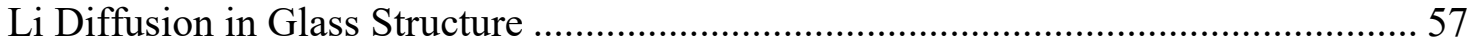

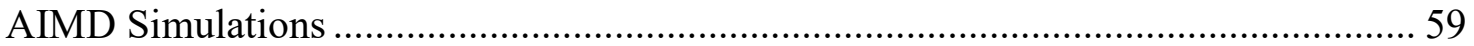

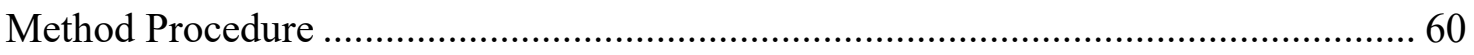

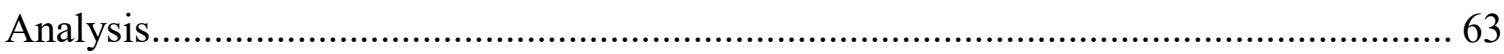

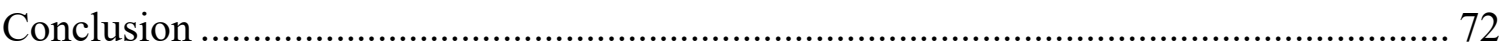

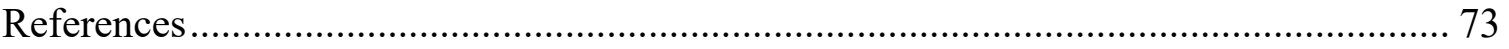

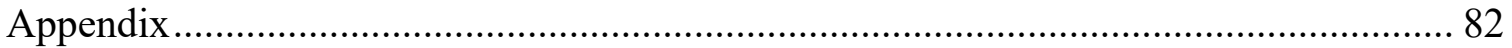




\section{LIST OF TABLES}

Table 1: Comparison between Experimental and Computational methods[15] ............... 9

Table 2: Comparison between Classical MD and DFT analysis ................................. 10

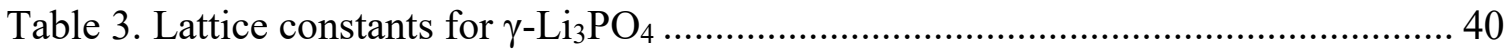

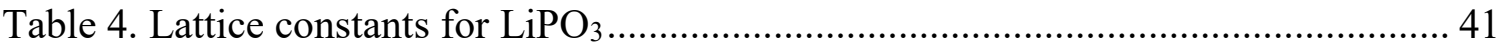

Table 5: Comparison of Energy barrier in different path in different crystal structure \&

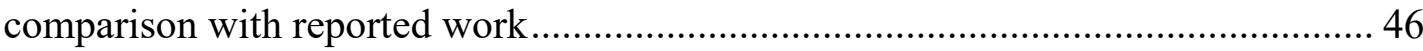

Table 6. Comparison of bond length over different volume.......................................... 54

Table 7. $4 \mathrm{~d} 1 \mathrm{~d}<\mathrm{LiO}>$ distance, $\mathrm{CN}$ of migrating Li with structure energy for each image

Table $8.2 \mathrm{c} 1 \mathrm{~d}<\mathrm{LiO}>$ distance, $\mathrm{CN}$ of migrating $\mathrm{Li}$ with structure energy for each image

Table 9: Energy barrier comparison with experimental work for pure Li2O-S2O5 and 60-

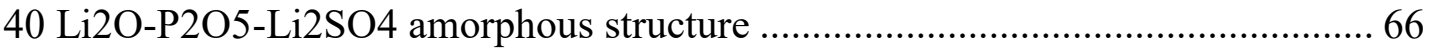

Table 10. Experimental result of conductivity and energy barrier for different composition [2] 


\section{LIST OF FIGURES}

Fig 1. Comparison of different battery technology with respect to volumetric energy

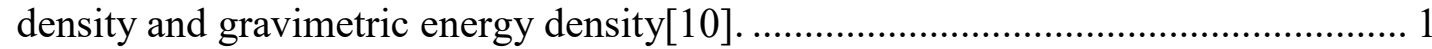

Fig 2. Diagram of a Li-ion battery. Li-ion diffuse from the anode to the cathode in discharge process and electron flow through the external circuit [15] ....................... 3

Fig 3. Workflow of energy calculation using Hartree-Fork Method ............................... 13

Fig 4. Energy vs Ecutoff curve to estimate favorable Ecutoff for calculation ................. 16

Fig 5. Energy calculation method using density functional theory ……......................... 20

Fig 6. The Nudge Elastic Band method. CI-NEB. An elastic band is formed between the five images. The initial images are placed on the interconnected line with an equal distance. The images are then moved keeping the spring force parallel to the path and component of the true force perpendicular to the path.

Fig 7. The length and time scales of different simulation and experimental techniques.

CMD: classical molecular dynamics; AIMD: ab initio molecular dynamics; CDFT: classical density functional theory; TDDFT, time-dependent density functional theory.[73]

Fig 8. Schematic illustration of the pseudopotential concept. The dash lines show the allelectron wave-function, $\Psi^{\wedge} \mathrm{AE}(\mathrm{r})$ while the solid lines show the corresponding pseudo-wave-function, $\Psi^{\wedge} \mathrm{PS}$ (r) given by the pseudopotential; the cutoff radius rc $\mathrm{m}$

Fig 9. Electron density map of diborane in the plane of the terminal hydrogen atoms .... 34

Fig 10. $2 \times 2 \times 1 \mathrm{Li}_{3} \mathrm{PO}_{4}$ supercell. Here red atoms are $\mathrm{O}$, violet indicates $\mathrm{P}$ and Green atoms are $\mathrm{Li}$

Fig 11. Relax method to get optimum energy structure................................................. 38

Fig 12. $\mathrm{LiPO}_{3}$ supercell showing chain of $\mathrm{Li}$ and the arrows shows the sites of interest for diffusion calculation ............................................................................................. 40 
Fig 13. Li-ion migration a) total energy profile of supercell in $1 d-2 c-3 d$ site migration b) cross-section of the migration process $\mathrm{c}$ ) Interaction of Li with oxygen in the migration process in $\mathrm{Li}_{3} \mathrm{PO}_{4}$ supercell. Green indicates $\mathrm{Li}$, Red indicates $\mathrm{O}$ atom.42

Fig 14. Li-ion migration a) total energy profile of supercell in $1 \mathrm{~d}-4 \mathrm{~d}$ site diffusion b) cross-section of the migration process $\mathrm{c}$ ) Interaction of Li with oxygen in migration time in $\mathrm{Li}_{3} \mathrm{PO}_{4}$ supercell

Fig 15. a. Possible Li diffusion paths in Li3PO4 crystal supercell. b. absolute energy profile for all of them $\mathrm{c}$ site in left, $d$ in right......................................................... 44

Fig 16. Li-ion migration a) total energy profile of supercell in Li16-Li14 site diffusion b) top view of the migration process c) aggregated snapshots of the migration process showing the interaction of $\mathrm{Li}$ with oxygen in the interim process in $\mathrm{LiPO}_{3}$ supercell.

Fig 17. Aggregated change in charge in all the images for $2 \mathrm{c}-1 \mathrm{~d}$ diffusion 47

Fig 18. Center of the tetrahedral Li(cyan, larger atom) indicate vacancy site. The surrounding highlighted atoms are experiencing a significant force due to the vacancy defect of $\mathrm{Li}$.

Fig 19. Change of Li-O and P-O bond with respect to the total volume change.

Fig 20. Energy barrier for different paths $1.2 \mathrm{c} 1 \mathrm{~d} 2.1 \mathrm{~d} 4 \mathrm{~d} 3.2 \mathrm{c} 3 \mathrm{~d}$................................ 50

Fig 21. Energy profile of $4 \mathrm{~d} 1 \mathrm{~d}$ and $2 \mathrm{c} 3 \mathrm{~d}$ paths in different structural volume................. 51

Fig 22. Energy vs $<$ Li-O $>$ bond length relation for $4 \mathrm{~d} 1 \mathrm{~d} \&$ 2c1d path ............................ 52

Fig 23. Change in $\mathrm{O}$ bond distance for each $\mathrm{Li}$ and $\mathrm{P}$ in $\mathrm{Li3PO} 4$ structure....................... 53

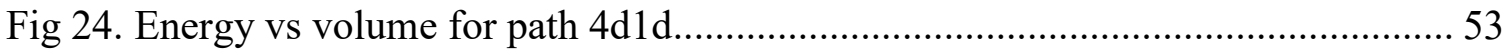

Fig 25. A sample Ionic bond energy vs inter-atomic distance plot ................................. 56

Fig 26. Li-ion migration a) energy profile in Li4-Li12 site migration b) top view of the migration process c) energy profile in Li14-Li12 site migration d) top view of the migration process in $\mathrm{LiPO}_{3}$ supercell 
Fig 27. a) $50 \% \mathrm{Li}_{2} \mathrm{O}-50 \% \mathrm{P}_{2} \mathrm{O}_{5}$ supercell containing interconnecting $\mathrm{P}-\mathrm{O}$ polyhedral cluster containing $24.59 \%$ volume of the total space. b) RDF analysis of crystal and amorphous structure showing the disappearance of long-range order.

Fig 28. a) Electron density map around $\mathrm{S}$ and $\mathrm{P}$ in $60 \%\left(\mathrm{Li}_{2} \mathrm{O}-\mathrm{P}_{2} \mathrm{O}_{5}\right) 40 \% \mathrm{Li}_{2} \mathrm{SO}_{4}$. b) Coordination number analysis of $\mathrm{P} \& \mathrm{~S} \mathrm{c}$ ) $\mathrm{CN}$ analysis of $\mathrm{Li}$ in both structures...... 62

Fig 29. Li migration for 2 random $\mathrm{Li}$, with $\mathrm{Li}-\mathrm{O}$ bond handover process in $\mathrm{LiPO}_{3}$ amorphous structure. Green indicates $\mathrm{Li}$ and red $\mathrm{O}$ atom.

Fig 30. LiPO3SO4 supercell showing a cluster of $\mathrm{P}-\mathrm{O}$ polyhedral (violet) and isolated SO tetrahedral (yellow). Theses structure consumes $15.344 \%$ of total supercell space.

Fig 31. Average MSD of a different element in Li2P205SO4 at 3000K for $12 \mathrm{ps.}$ 67

Fig 32. Average MSD of different element in Li2P2O5 at 3000K for 3 ps.

Fig 33. Diffusivity vs $1 /$ T graph. Showing comparison of energy barrier with experimental result 68

Fig 34. Li migration with $\mathrm{Li}-\mathrm{O}$ bond handover process in $\mathrm{LiPO}_{3} \mathrm{SO}_{4}$. The green mesh shows the movement of $\mathrm{Li}$ and the red-pink bar showing the dynamic $\mathrm{Li}-\mathrm{O}$ bond created in the intermediate stages. It also shows the $\mathrm{O}$ atoms (red mesh) are localized during the migration a) a long migration where several $\mathrm{O}$ contribute to the migration b) site to site migration contributed by only 2 oxygen atom.

Fig 35. a) Vibration frequency of $\mathrm{Li}$ (green), $\mathrm{O}$ (orange), $\mathrm{P}$ (gray), $\mathrm{S}$ (yellow) in $\mathrm{LiPO}_{3}$ amorphous medium and b) in $\mathrm{Li} 2 \mathrm{P} 2 \mathrm{O} 5 \mathrm{Li}_{2} \mathrm{SO}_{4}$. Total $\mathrm{Li}$ frequency band shifted to the left in S mix one. 


\section{INTRODUCTION}

\section{Li-ion Battery}

Anticipating the decline of conventional fossil fuel reserves and facing the discontinuous nature of power generation from alternative renewable energy, storage system research is of prime concern [3]-[7]. Numerous work has been done in this field to meet ever-growing expectations of reliability, durability, and capacity constraints of storage devices. Over the past few decades, Li-ion batteries have been proven to be superior to Lead- or Sodium-ion batteries. Fig 1 clearly shows the superiority of Li-ion battery over other conventional ionic battery in terms of energy density. Conventional Lead-acid batteries, for example, tends to have a short lifetime, while sodium sulfur and sodium-metal halide batteries have safety risk due to the thermal and mechanical stability of $\beta 00$-alumina tube at battery operating temperature [8], [9].

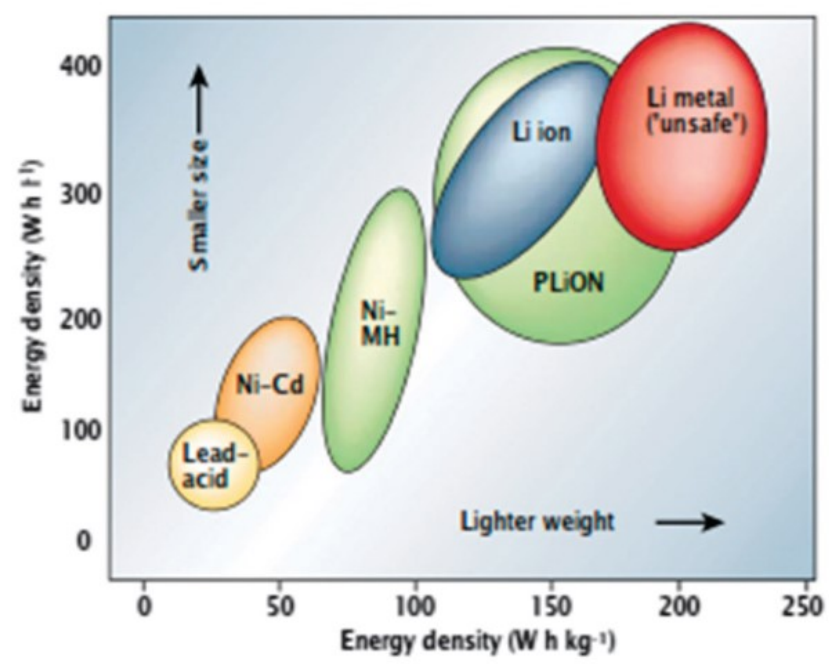

Fig 1. Comparison of different battery technology with respect to volumetric energy density and gravimetric energy density[10]. 
In every electrochemical cell, there are two electrodes (namely, anode and cathode) and an electrolyte separating the electrode from direct contact, as shown in Fig 2. The anode has an excess of ions, while cathode works as a sink of these excess ions. Both the electrodes have to be a good electrical conductor, whereas the electrolyte has to be a poor electron conductor but a good conductor of the ion. In the discharge process, oxidization reaction occurs in the cathode $\left(\mathrm{Li}=\mathrm{Li}^{+}+\mathrm{e}^{-}\right)$and generated $\mathrm{Li}$-ions travel through the anode to the cathode via the electrolyte. Meanwhile, the electron generated from the oxidization reaction travels from the cathode to anode through the external circuit supplying the required energy to do the work. During the charging process, this cycle is reversed via external potential applied to the electrode, resulting in accumulation of $\mathrm{Li}$ in the anode. Typically, the cathode consists of ion-rich crystalline material while the commercial anodes tend to be carbon- or silicon-based. However, being electrically insulating, the electrolyte needs to be a solution or a glass-type material of organic or inorganic ion-rich material. Moreover, as Li is the lightest and most electropositive element, it can show very high potential in $\mathrm{Li} / \mathrm{Li}+$ reaction for different compounds. For example, Li-ion in $\mathrm{LiMnPO}_{4}, \mathrm{LiCoPO}_{4}$, and $\mathrm{LiNiPO}_{4}$ can deliver 4.1 V, $4.8 \mathrm{~V}$ and $5.1 \mathrm{~V}$ potential, respectively [11]-[13]. This is to be compared with the early introduction from Sony, which introduction of $\mathrm{LiCoO}_{2}$ has a limited capacity of $\sim 140 \mathrm{mAhg}^{-1}$ at $3.7 \mathrm{~V}$. To increase the energy density even further, layered oxides $\mathrm{Li}[\mathrm{Li}, \mathrm{Mn}, \mathrm{Ni}, \mathrm{Co}] \mathrm{O}_{2}$ have been considered for higher capacity values of $\sim 250 \mathrm{mAhg}^{-1}[14]$. Along with its high potential, Li also shows high diffusivity, thermal stability and low energy barrier which make it a superior charge transport medium compared to other existing options. 


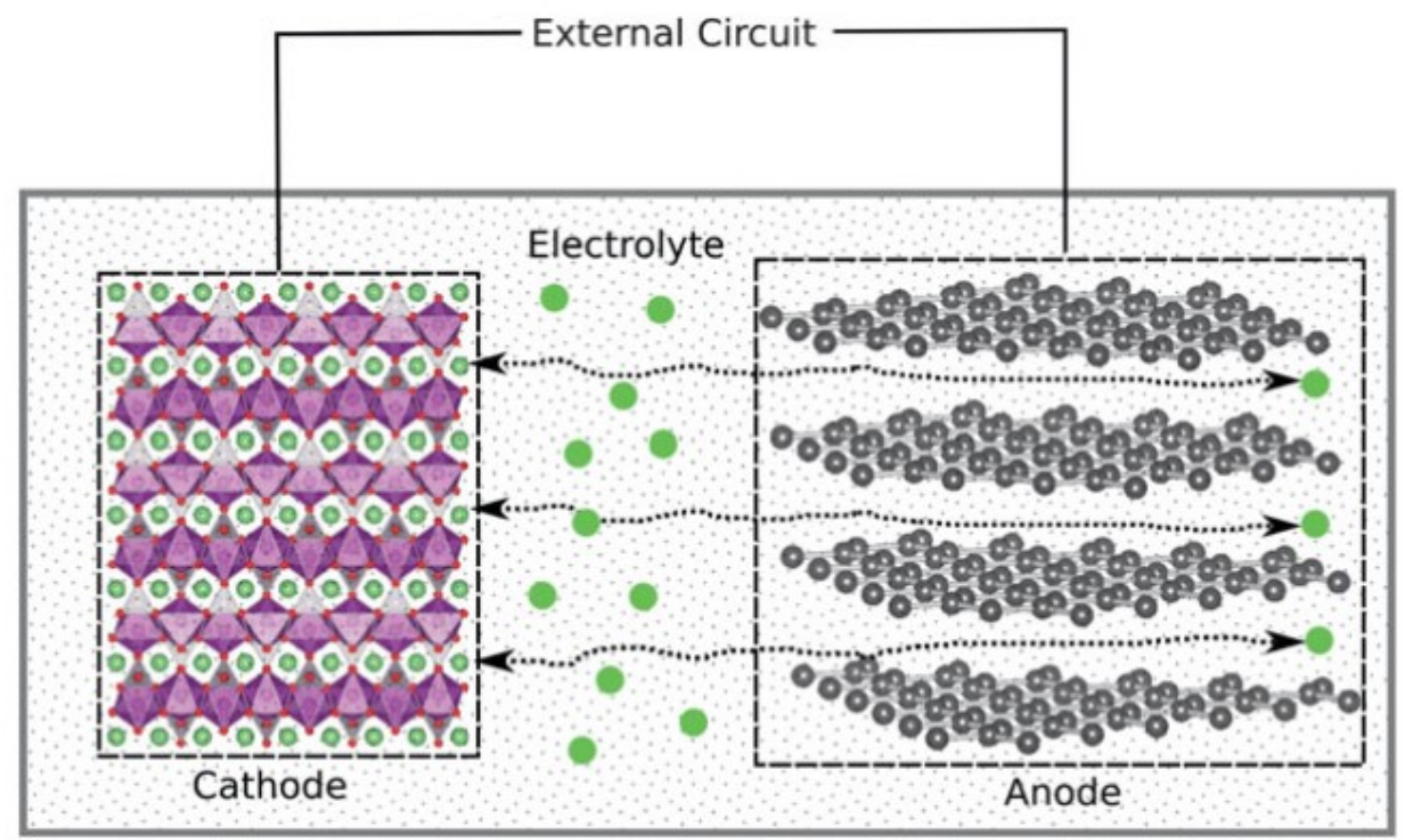

Fig 2. Diagram of a Li-ion battery. Li-ion diffuse from the anode to the cathode in discharge process and electron flow through the external circuit[15].

\section{Solid State Electrolyte}

For achieving higher electro-potential and charge capacity, not only the conductor, but also a high-performance electrolyte is essential. However, in developing the next generation of Li-ion batteries, electrolyte becomes the main obstacle. Recently, most of the works on battery electrolyte are focused on solid-state electrolyte (SSEs), which is produced by transforming liquid electrolyte batteries to solid-state lithium batteries (SSLiBs). These solid-state lithium electrolyte (SSEs) can solve some age-old problem of liquid batteries such as limited operating voltage, physical, thermodynamics, electrochemical safety issues, chemical stability with electrode material, poor cycling property, poor ion conductivity in room temperature, and low activation energy (Ea) for use over a broad range of temperature. On the other hand, they are prone to oxidization 
combustion, leaking, or internal shorting[16], inexpensive and environmentally friendly thin film preparation method and also inert to metallic Li conductor and resist to grow dendrite[16]. Along with these, solid electrolytes allow lighter and more flexible design criteria in the shape and size. A range of SSEs has been investigated from, among others, gelled polymers, solvent-free polymers, inorganic crystalline compounds, and inorganic glasses. Some recent examples include: $\mathrm{LiBH}^{4}$ [17]; $\mathrm{Li}_{3} \mathrm{~N} ; \mathrm{Li}_{4} \mathrm{SiO}_{4}$ [18], [19]; perovskites [20], [21]; Garnet-type $\mathrm{Li}_{\mathrm{x}} \mathrm{AyB}_{3-\mathrm{y}} \mathrm{M}_{2} \mathrm{O}_{12}(\mathrm{x}=3,5,6,7 ; \mathrm{y}=0,1 ; \mathrm{A}=\mathrm{Mg}, \mathrm{Ca}, \mathrm{Sr}, \mathrm{Ba} ; \mathrm{B}=$ La, Y, Pr, Nd, Sm-Lu; M = Nb, Ta, Sb, Zr, Sn, Te) [22]-[24], $\mathrm{Li}_{7} \mathrm{La}_{2.75} \mathrm{Ca}_{0.25} \mathrm{Zr}_{1.75} \mathrm{Nb}_{0.25} \mathrm{O}_{12}\left(\sigma=2.2 \times 10^{-4} \mathrm{~S} / \mathrm{cm}\right)$ [25], $\mathrm{Li}_{2} \mathrm{SO}_{4}-\mathrm{Li}_{2} \mathrm{O}_{-} \mathrm{P}_{2} \mathrm{O}_{5}$ [26], $\mathrm{Na}_{2} \mathrm{O}-$ $\mathrm{P}_{2} \mathrm{O}_{5}$ [27], and $\mathrm{K}_{2} \mathrm{O}-\mathrm{P}_{2} \mathrm{O}_{5}[2]$.

Recently, polyethylene oxide (PEO) and related polymer mixed with Li salt draw attention due to its high conductivity in higher temperature. At the same time, these materials also show conductivity at a lower temperature as segmental chain motion diminishes, which is the dominant factor for high conductivity. However, poly vinylidene fluoride (PVDF)-based material shows ionic conductivity up to $0.1 \mathrm{Sm}^{-1}$ at room temperature[28], [29].

Among these, most of the highly conductive compounds show degrading electrochemical stability with electrodes. For example, $\mathrm{La}_{(2 / 3)-x} \mathrm{Li}_{(1 / 3)+x} \mathrm{TiO}_{3}$ (LLT) shows high conductivity of $10^{-3} \mathrm{~S} / \mathrm{cm}$, even though it's coupling with Li conductors is poor [20]. Their grain boundary offers high resistance to total ionic conductivity at low temperature. Another highly conductive material, $\mathrm{Li}_{3} \mathrm{~N}$ electrolyte shows low decomposition voltage of $0.44 \mathrm{~V}$ at room temperature. Similarly, $\mathrm{Li}-\beta$ alumina also shows isotropic conductivity but sensitive to moisture and carbon dioxide[18]-[20], [30]. 
These types of fundamental issues underlie the quest to fully understand the behavior of solid batteries, especially the area of the electrochemical interface. The interaction between solvent and lithium salts during discharge-charge in the cell may affect the performance of the batteries. Decomposition of $\mathrm{LiPF}_{6}$ creates a thick layer of $\mathrm{LiF}$ in the electrode material, creating a barrier to an electrode-electrolyte system which serves as an additional internal resistance to Li-ion flow and results in a decrease of output voltage[31]. The particular microscopic mechanism on the SEI interface is still not resolved because of the difficulty in situ observation in the electrode-electrolyte interface in operating time. The composition of the SEI can be a vital factor to interface mechanism. For example, about 5-10\% presence of vinylene carbonate (VC) in ethylene carbonate (EC) solvent improves the irreversibility capacity at the charging cycle in Liion polymer cell[32], [33].

There are several attempts reported to engineer SSE material to increase ion mobility. Li-ion doped plastic crystalline matrices are reported stable in $5 \mathrm{~V}$ and on interest due to the plastic crystal matrices vibrational characteristics. $\mathrm{LiClO}_{4}$ doped PEO with inorganic hybrid poly(cyclotriphosphazene, 40-sulfonyldiphenol) (PZS) as filter shows higher conductivity[34] compared to ceramic fillers like SiO2. Brittle superionic glass also shows potential for higher diffusion material candidacy. LiGaS and LiSiS known as LISICON are recently discovered with a higher electrochemical stability[35], [36]. A lot of research is going on these $\mathrm{Li}_{2} \mathrm{~S}-\mathrm{P}_{2} \mathrm{~S}_{5}$ type of electrolyte systems. Existing solid electrolytes can be divided into 3 groups: (i) inorganic SE (ISEs), (ii) composite solid electrolytes (CSEs), and (iii) solid polymer electrolytes (SPEs). This work is based 
on ISE materials and understands the Li conductivity and influence of Oxygen on the diffusion of Li-ion.

On the other hand, several experimental works have been done on conductivity and stability of $\mathrm{Li}_{3} \mathrm{PO}_{4}$ [37], $\mathrm{LiPO}_{3}$, and its corresponding glass form $\mathrm{Li}_{2} \mathrm{O}-\mathrm{P}_{2} \mathrm{O}_{5}$ \& $\mathrm{xLi}_{2} \mathrm{OP}_{2} \mathrm{O}_{5}-(1-\mathrm{x}) \mathrm{L}_{2} \mathrm{O}-\mathrm{SO}_{4}[38]$ reports activation energy of $1.4 \mathrm{eV}$ for polycrystalline $\mathrm{LiPO}_{3}$ with de conductivity of $2.5 \times 10-8 \mathrm{~S} / \mathrm{cm}$ at $280 \circ \mathrm{C}$, which can be enhanced by four orders of magnitude in its corresponding glass form. $\mathrm{Li}_{2} \mathrm{O}-\mathrm{P}_{2} \mathrm{O}_{5}$ is rich on $\mathrm{Li}$ and highly stable in both crystalline and glass $\mathrm{Li}_{2} \mathrm{O}-\mathrm{P}_{2} \mathrm{O}_{5}$ form. $\lambda$ - $\mathrm{Li}_{3} \mathrm{PO}_{4}$ crystal is also important because of its reported stability properties with Li-based compounds [39] with its layered order of $\mathrm{Li}$ indicating a potential $\mathrm{Li}+$ migration channel. The aim of this study is to computationally understand the behavior of $\mathrm{Li}$ transport in atomic level for $\mathrm{LiPO}_{3}$ crystalline media and compare with the glass counterparts.

\section{Theoretical Approaches}

Recently theoretical approaches are used widely to design and understand material properties in every aspect of research. Theoretical approaches provide a cost-efficient process to understand the atomic mechanism, complement experimental analysis, screen and predict properties. Table 1 provides a comparison between the Computational and experimental approaches, their aim and analysis technics. The theoretical calculation has been recently used to predict $\mathrm{Li}+$ ion conduction process [40]-[43], but most of the reports fail to explain the actual mechanism and predict conduction pathway [38]. Theoretical analysis has been practiced in every aspect of the battery material to understand and link up various aspects like diffusion in electrode or electrolyte, an 
electrical response, interfacing between electrode and electrolyte, the effect of chargedischarge process on the battery structure etc. There is mainly two form of theoretical approach been practiced on the battery material: interatomic potential based classical analysis and electronic structure methods (mainly density functional theory DFT). However, also Monte-Carlo simulation is also a subcategory of investigating Li-ion battery. A summary of comparison is provided in Table 2. The interatomic potential method is based on the potential model developed on the classical understanding of the interaction between different atoms and calculating the total energy. For polar models, the interaction can be summarised into a long-range order Coulombic interaction, a shortrange order repulsion force between charges and van der walls force due to electron charge clouds. A well-known Buckingham potential can be expressed as:

$$
U_{i j}(r)=\frac{q_{i} q_{j}}{r}+A_{i j} \exp \left(-\frac{r}{\rho_{i j}}\right)-\frac{C_{i j}}{r^{6}}
$$

Where $U_{i j}$ is the potential energy between $\mathrm{i} \& \mathrm{j}$ ions with a separation distance r. q is the charge of the ions. This model is effective for calculating different dynamic bulk properties and dielectric properties. It might look the potential is suitable for ionic system but Catlow et al argued [44], [45] that this can be applied irrespective of the material type. However much better type of interatomic potential also depends on the characteristics of the structure and there are several of them already developed up to now. For example, for Phosphate and Silicates, the pair potential is still provides reasonable accuracy also an inclusion of angular dependency can increase the accuracy to a higher level[44], [46]. Minimizing of energy can be done by searching to the potential energy surface of the structure and using routine steps of reducing tension effect on the particles. The total process is done using a periodic boundary condition and with a enclosed 
simulation box. The periodic boundary condition is applied considering the same activity of the box is imaged in every direction of the physical space with a period of the box dimension with an infinitely long extension. The MD technique is consist of solving Newton's equation of motion to predict time dependent trajectories and can be employed in a large ensembles of particle in a finite temperature and pressure. Employing the method on Li ion material we can extract the information of ion mobility in a certain environment and compare different characteristics like diffusivity and conductivity and closely observe the behaviours which is not yet possible in the most sophisticated equipment available up to now. GULP, LAMMPS, DL_POLY is widely used codes for classical MD analysis.

In the case of crystal electrode, because of its periodicity, probable hopping paths can be identified by considering the vacancy activation energy of various sites, with the lowest activation energy trajectory being the most probable hopping path [47]-[50]. On the other hand, because of lack of periodicity and long-range order, it is hard to predict probable hopping path, making crystal method unsuitable in the amorphous medium. In several studies [51], [52] molecular dynamic approach is used to identify $\mathrm{Li}+$ ion diffusivity in amorphous medium but the mechanism is still inconclusive. However, their calculation shows an important observation: in between a total site to site migration, $\mathrm{Li}$ oscillate in localized position. In an early work, bond valence sum (BVS) is used to study Li-ion conduction in $\mathrm{Li}_{5} \mathrm{La}_{3} \mathrm{M}_{2} \mathrm{O}_{12}(\mathrm{M}=\mathrm{Nb}, \mathrm{Ta})$. They found, Li-ion conductivity is anisotropic and mostly around the MO6 octahedron region[53]. They have also reported the unusual behavior of the BVS in $\mathrm{Li}_{5} \mathrm{La}_{3} \mathrm{M}_{2} \mathrm{O}_{12}$ crystal data and optimized $\mathrm{Li}$ and $\mathrm{O}$ position using the global instability index and minimization of energy structure using 
bond valence mismatch minimization procedure. Also, several ab initio analyses have been used to understand Li structural property in a different structure. Xu et al. used AIMD to analyze $\mathrm{Li}_{3} \mathrm{La}_{3} \mathrm{Te}_{2} \mathrm{O}_{12}, \mathrm{Li}_{5} \mathrm{La}_{3} \mathrm{Nb}_{2} \mathrm{O}_{12}$, and $\mathrm{Li}_{7} \mathrm{La}_{3} \mathrm{Zr}_{2} \mathrm{O}_{12}$ structure and found $\mathrm{Li}_{3}$ and $\mathrm{LI}_{5}$ phase supports high Li occupancy in the tetrahedral sites[54]. This work is focusing on $\mathrm{Li}+$ ion migration analysis in both crystalline $\mathrm{Li}_{3} \mathrm{PO}_{4} \& \mathrm{LiPO}_{3}$ structure and glass $\mathrm{Li}_{2} \mathrm{O}-\mathrm{P}_{2} \mathrm{O}_{5} \& 0.4\left(\mathrm{Li}_{2} \mathrm{O}-\mathrm{P}_{2} \mathrm{O}_{5}\right)-0.6\left(\mathrm{Li}_{2} \mathrm{O}-\mathrm{SO}_{4}\right)$ structure-type solid-state electrolyte. I have shown the interaction of $\mathrm{Li}$ in the structure is predominantly controlled by surrounding oxygen environment. I have calculated the activation energy and predicted the optimal sulfur composition.

Table 1: Comparison between Experimental and Computational methods[15]

\begin{tabular}{|c|c|c|}
\hline & Computational & Experiment \\
\hline Method & $\begin{array}{l}\text { Potential based method (Monte-Carlo } \\
\text { method, classical molecular dynamics) } \\
\text { Electronic Structure method (DFT, } \\
\text { AIMD) }\end{array}$ & $\begin{array}{l}\text { Material synthesis } \\
\text { X-ray diffraction } \\
\text { Nuclear magnetic resonance } \\
\text { Neutron diffraction } \\
\text { Electron microscopy } \\
\text { Various spectroscopy } \\
\text { Electrical measurement }\end{array}$ \\
\hline Aims & $\begin{array}{l}\text { Complement experimental analysis } \\
\text { Screening and predict properties } \\
\text { Understand atomic properties } \\
\text { Elucidate atomic-scale feature difficult } \\
\text { to understand from experimental } \\
\text { methods }\end{array}$ & $\begin{array}{l}\text { Cell voltage and phase changes } \\
\text { with cycles } \\
\text { Li diffusion paths } \\
\text { Defect analysis } \\
\text { Interface analysis } \\
\text { Crystal structure and stability }\end{array}$ \\
\hline
\end{tabular}


Table 2: Comparison between Classical MD and DFT analysis

\begin{tabular}{|l|l|l|}
\hline Context & MD & $\begin{array}{l}\text { Electronic Structure (DFT, } \\
\text { AIMD) }\end{array}$ \\
\hline Method & $\begin{array}{l}\text { Classical Newtonian } \\
\text { mechanics }\end{array}$ & Quantum mechanics \\
\hline Accuracy & Moderate & Highly accurate \\
\hline $\begin{array}{l}\text { Potential file } \\
\text { availability }\end{array}$ & low & $\begin{array}{l}\text { Available for all-atom in periodic } \\
\text { series }\end{array}$ \\
\hline Computational cost & Inexpensive & Expensive \\
\hline $\begin{array}{l}\text { Detailed electronic } \\
\text { structure information }\end{array}$ & Not available & Available \\
\hline Sample size & $10^{6}$ & $100-200$ \\
\hline
\end{tabular}

Over last decade, atomistic modeling to understand and evaluate Li-diffusion in the electrolyte in Li-ion batteries are getting popular. In this work, Li+ migration barrier in the crystal is analyzed using electronic structure density functional theory model (DFT) and ab initio molecular dynamics calculation is used for the case of an amorphous structure which is described explicitly in the following section.

\section{Density Functional Theory (DFT)}

Density functional theory (DFT)[55] calculation is getting popular due to its highly accurate predictability to the material characterization and modeling of a new type of inorganic materials by calculation of ground state structure of the material. DFT works as a bridge to explain and understand different experimental results such as geometrical properties, electrical characteristics, physical characteristics etc. with the theoretical 
approach by building up the similar environment of the region of interest with appropriate conditions. However, DFT has also been used to model a new type of prospective material and understand the behavior of a specific regional characteristic of material where experiments cannot be performed[56]. Higher accuracy of DFT method opens the opportunity to prediction based material research.

DFT is based on solving Schrodinger equation. For many electron environments, Schrodinger equation became most complex and difficult to solve:

$$
\begin{aligned}
H=\sum_{j=1}^{N}\left(-\frac{\hbar^{2}}{2 M_{j}}\right) \nabla_{R_{j}}^{2} & \frac{1}{2} \sum_{j}^{N} \sum_{i=1}^{N}\left(\frac{Z_{i} Z_{j} e^{2}}{\left|R_{i}-R_{j}\right|}\right) \\
& +\sum_{j=1}^{n}\left(-\frac{\hbar^{2}}{2 m}\right) \nabla_{r_{j}}^{2}+\frac{1}{2} \sum_{j=1}^{n} \sum_{i=1}^{n}\left(\frac{e^{2}}{\left|r_{i}-r_{j}\right|}\right)+\sum_{j=1}^{N} \sum_{i=1}^{n}\left(\frac{Z_{j} e^{2}}{\left|r_{i}-R_{j}\right|}\right)
\end{aligned}
$$

Where $\mathrm{R}_{\mathrm{i}}$ is the position of nuclei, $\mathrm{r}_{\mathrm{j}}$ are the position of electrons, $\mathrm{eZ}$ are a charge of nuclei and $\mathrm{M}$ is the mass of the nuclei. There is no direct solution proposed for this many body systems and a generalization is a must. The researcher has been proposed several approximations solve this problem and there are three notable approximations exists. Born-Oppenheimer considered nuclei's as stationary and used non-relativistic Schrodinger equation to calculated reduced electron wave-function from the system. Doing this for all coordinate the minimum energy configuration can be achieved. However, in the approximation, the overall wave function is treated as a product of single-particle wave-function. Still, it was difficult to solve the wave-function for the $\mathrm{n}^{\text {th }}$ atom. Another approximation was proposed by Hartree and Fock in 1920. They proposed an $\mathrm{N}$-atom system can be represented by a Slater determinant of $\mathrm{N}$ spin electrons orbitals instead of the product of their individual wave-function. 


$$
\Psi=\frac{1}{\sqrt{N !}}\left|\left(\begin{array}{ccc}
\Phi_{1}(1) & \cdots & \Phi_{N}(1) \\
\vdots & \ddots & \vdots \\
\Phi_{1}(N) & \cdots & \Phi_{N}(N)
\end{array}\right)\right|
$$

Where, $\Phi_{1}(1), \Phi_{2}(2) \ldots \ldots \Phi_{N}(N)$ are electron wave-function. This solves the problem of antisymmetric since exchanging any two row of the determinant causes change in sign where as if the resultant wave-function is a product of all separate electron wave-function, they will not yield change in sign when exchanged between each other. Also this determinant form holds that two electron can have the same wave-function. Upon introducing the Slater determinant, the approximation becomes a single particle problem. A very important factor of the Hartree approximation is the probability of finding an electron in a particular point is independent of any other electron probability at that point. And hence the electrons orbitals are independent of each other. However, this is one of the drawback of Hartree method. They fail to incorporate the exchange energy in the total energy. The energy will be simply:

$$
E=\frac{\psi^{*} H \psi d r}{\int \psi^{*} \psi d r}
$$

In the slater wave-function, the total energy holds below relation:

$$
\begin{gathered}
\langle\psi|H| \psi\rangle=\sum_{i=1}^{N} E_{i}+\sum_{i<j}\left(C_{i j}-E_{i j}\right) \\
E_{i}=\left\langle\varphi_{i}|h| \varphi_{i}\right\rangle \\
C_{i j}=\left\langle\varphi_{i} \varphi_{j}\left|V_{i n t}\right| \varphi_{i} \varphi_{j}\right\rangle \\
E_{i j}=\left\langle\varphi_{j} \varphi_{i}\left|V_{i n t}\right| \varphi_{i} \varphi_{j}\right\rangle
\end{gathered}
$$


Where $C_{i j}$ is the Coulomb integral of $I \& j^{\text {th }}$ orbital and $E_{i j}$ is the exchange integral between I \& $\mathrm{j}^{\text {th }}$ orbital electrons. The aim is to minimize the expected value of the Hamiltonian. A typical Hartree energy approximation tree is shown in Fig 3

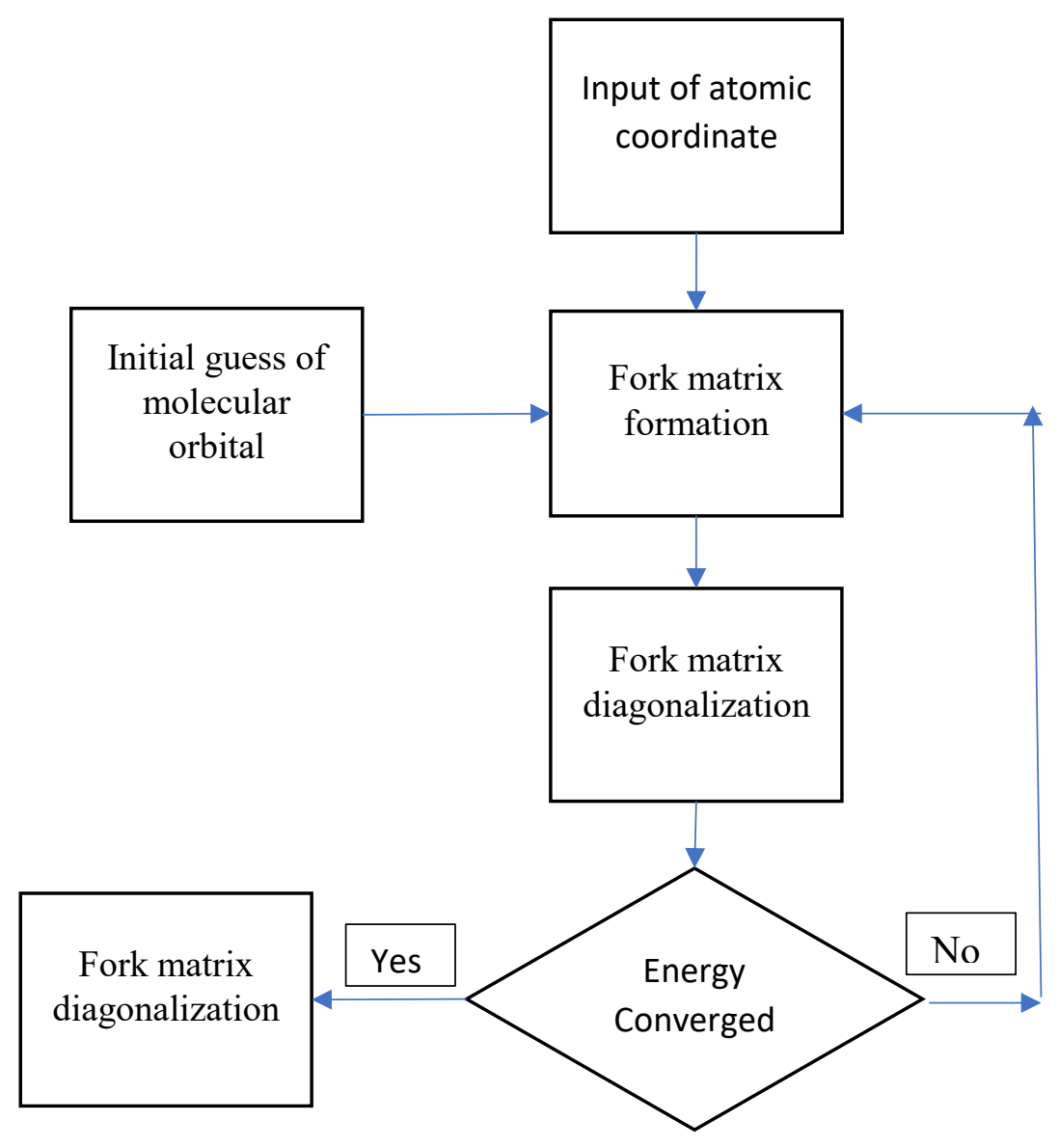

Fig 3. Workflow of energy calculation using Hartree-Fork Method

Another approach is Linear combination of atomic orbitals (LCAO method). In LACO method the molecular orbitals are written as a linear weighted summation of individual atomic orbitals: 


$$
\phi_{i}=\sum_{i} C_{r i} x_{r}
$$

An orbital is a one-electron function. Most of the recent code use the AO as atom centered Gaussians and some of the older code package use Slater functions. For Stalter orbitals:

$$
\phi_{a b c}^{S T O}(x, y, z)=N x^{a} y^{b} z^{c} e^{-\zeta r}
$$

Where $\mathrm{a}, \mathrm{b}, \mathrm{c}$ controls the angular momentum of the orbital, $\varsigma$ controls the width of the orbital. $\mathrm{N}$ is constant.

For the case of Gaussian orbitals representation are similar:

$$
\phi_{a b c}^{G T O}(x, y, z)=N x^{a} y^{b} z^{c} e^{-\zeta r^{2}}
$$

The STO provides more accurate electron orbital than GTO but more computationally expensive. People use a combination of n Gaussian to approximate STO which is known as "STO-nG":

$$
\phi_{a b c}^{C G T O}(x, y, z)=N \sum_{i=1}^{n} c_{i} x^{a} y^{b} z^{c} e^{-\zeta r^{2}}
$$

Density functional theory comes from Hohenberger-Known theorem. Instead of focusing on individual electron orbitals as described earlier, DFT focuses on the electron density. Hence the many body wave-function methods transformed to solve electron density in the stricter. Hohenberg-Kohn theorem suggests the total ground state energy of many electron structures is a functional of the electron density $(\rho)$ of the system. Khon also proved the correct ground state energy can be acquired for which the energy is minimized. The relation of electron density (p) and Energy (E) is represented as bellow:

$$
\left(-\nabla^{2}+V_{H}[\rho(r)]+V_{N}(r)+V_{X C}[\rho(r)]\right) \psi_{i}(r)=E_{i} \psi_{i}(r)
$$


Where $V_{H}$ is the Hartree term representing the electrostatic energy of moving electron due to the interaction of all another electron in the system. $V_{N}$ is the energy of all the nuclei, $\mathrm{V}_{\mathrm{XC}}$ is the electron exchange-correlation term. $\Psi$ is the wave function of the electron density. There is no orbital concept in DFT and the way to solve the Khon-sham equation is to represent the wavefunction using a basis set to solve the above equation. A plane wave is one of the solutions to keep thinks mathematically simple and it completely spans in Hilbert space. However, the plane wave has the characteristics to span in all space equally and not localized. This is useful in guessing the first set of wave-function without any prior knowledge of the electron wave-function behavior in any structure. The equal distribution nature results in lacking electron density whereas there is a high electron density and it leads to the cubic scaling of plane wave DFT calculation with system size[57], [58]. Hence several work was done to focus on achieving basis set with linear to the system size[59]-[61].

$$
\varphi_{\alpha}(r)=\frac{1}{\sqrt{\Omega}} \sum_{G} c_{i}(G 1) e^{i G 1 . r}
$$

Where $\mathrm{G} 1=\mathrm{G}+\mathrm{k}$ is a vector in a reciprocal lattice. For a lattice with basis set a1, $\mathrm{a} 2, \mathrm{a} 3$, the $\mathrm{G}$ in the reciprocal lattice is:

$$
\begin{gathered}
G=i \cdot b_{1}+j \cdot b_{2}+k \cdot b_{3} \\
b 1=2 \pi \frac{a_{2} \times a_{3}}{a_{1} \cdot a_{2 \times} a_{3}} \\
b 2=2 \pi \frac{a_{3} \times a_{1}}{a_{1} \cdot a_{2 \times} a_{3}} \\
b 3=2 \pi \frac{a_{1} \times a_{2}}{a_{1} \cdot a_{2 \times} a_{3}}
\end{gathered}
$$


Where b1, b2, b3 are the basis set at reciprocal lattice space. An infinite sum of the basis set will result in the correct wave-function. But there is a cut-off we can choose to keep the computational cost in a controlled region and controlling parameter is $\mathrm{G}_{\max }$. The kinetic energy of the plane wave is related to the $G$ vector as:

$$
\begin{aligned}
-\frac{1}{2} \nabla^{2} \varphi_{G}(r) & =\frac{1}{2}\|G\|^{2} \varphi_{G}(r) \\
E_{\text {cutoff }} & =\frac{G_{\max }{ }^{2}}{2}
\end{aligned}
$$

To choose an $E_{\text {cutoff }}$ for a particular structure, we need to calculate the energy vs $E_{\text {cutoff }}$ plot and as soon as the graph flats down (Fig 4), we can use that particular cut-off for the calculation.

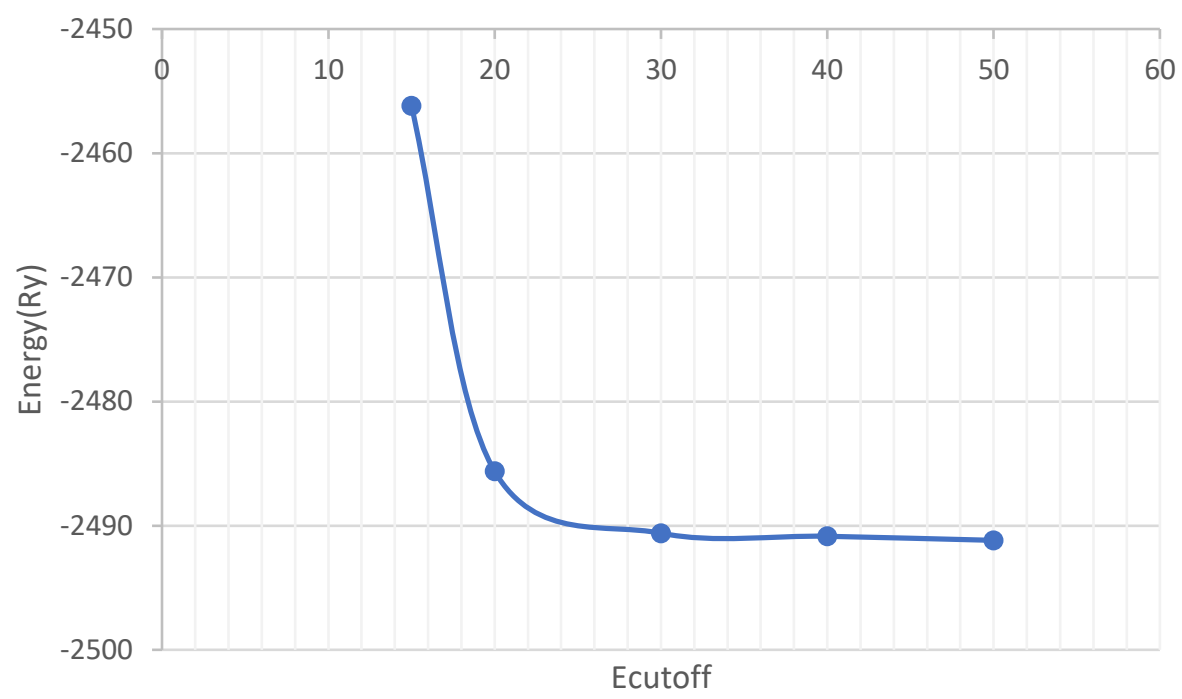

Fig 4. Energy vs Ecutoff curve to estimate favorable Ecutoff for calculation

For bulk calculation, periodic boundary condition is applied. However, for a large number of sample, there is an infinite number of electron contributing to the wave- 
function which is not trivial to solve. Bloch theorem suggests we can consider the electrons in the unit cell to solve the bulk system. From Bloch's theorem we know eigenstate of the periodic potential system can be written as:

$$
\psi_{j, k}(r)=u_{j}(r) e^{i k . r}
$$

Where ui(r) is the periodic potential, $u_{i}(r+l)=u_{i}(r) ; 1$ is period. $\mathrm{K}$ is the wave vector confined to the first Brillouin Zone. Doing Fourier transformation of $\mathrm{u}$, we get the final wave-function:

$$
\psi_{j, k}(r)=\sum_{G} c_{j k+G} e^{i(k+G) \cdot r}
$$

This is same as eqn. here also, infinite number electron creates an infinite number of $\mathrm{k}$ to represent $\psi$. For each $\mathrm{k}$ point, there is a limited number of occupied eigenstates. To get the approximate $\psi$, we can take a finite number of $\mathrm{k}$ point with little distortion of the $\psi$ and hence energy. On the other hand, wave-function of a reciprocal space can be represented by single k-point.

One of the most challenging parts of solving Khon-sham equation is predicting the $\mathrm{V}_{\mathrm{Xc}}$. There is no exact solution but there are a number of approximation exist. Local density approximation (LDA) is one of the widely used one. In this approximation, the potential is being calculated assuming a uniform electron gas surrounds each point not considering the derivatives of the density of the Kohn-Sham orbitals. A local density approximation for the exchange-correlation energy can be written as:

$$
E_{X C}^{L D A}|\rho|=\int \rho(r) \epsilon_{x c}(\rho) d r
$$

Where $\epsilon_{x c}$ is the exchange-correlation energy per particle corresponds to electron density of $\rho$. This method yields best result for the case of metallic type material. 
However, for the case of non-metallic material for example oxides and salts electrons shows localized characteristics. For this kind of material LDA, approximation results in several misoutcomes for example overestimation of the binding energy, inaccurate lattice constant etc. For these kinds of material generalised gradient approximation (GGA) method is developed. In GGA, the gradient of the electron density is expanded to account the non-homogeneity of electron in space. This method perform correction to the farther away electron density from the coordinate and correct the overestimation of delocalization of valance electron and stabilize the ground state for Mott insulators[62] and other non-metallic type materials. GGA has below form.

$$
E_{X C}^{G G A}[n \uparrow, n \downarrow]=\int \epsilon_{X C}\left(n \uparrow, n \downarrow, \nabla_{n \uparrow}, \nabla_{n \downarrow}\right) n(r) d^{3} r
$$

Using this approximation, good geometrical and ground state energy results have been reported. Over-delocalization of electrons may lead to introduce defective exchange and correlation interaction in the $\mathrm{XC}$ functional which may include electron selfinteraction Hartree term and result in an overestimation of energy. The extra energy term is coming from the electron self-repulsive force of the shared charge from the total electron cloud. This effect is far more prominent for the transition state elements. There are several methods proposed to fix this problem. Namely, DFT+ Dynamical Mean Field Theory (DFT+DMFT)[63], Reduced Density Matrix Functional Theory (RDMFT)[64] are two notable example. Even though this method shows significant correction of the addressed problem but computationally they are very expensive. Recently DFT $+U$ $(\mathrm{LDA}+\mathrm{U}$ or $\mathrm{GGA}+\mathrm{U})$ is being used to approximate DFT functional for the strongly correlated electronic state of systems whereas another valence electron can be 
approximated by standard approximation. In this method the total energy of the system can be written as:

$$
E_{L D A+U}[\rho(r)]=E_{L D A}[\rho(r)]+E_{H u b}\left[\left\{n_{m m}^{I \sigma}\right\}\right]-E_{d c}\left[\left\{n^{I \sigma}\right\}\right]
$$

Here $E_{L D A}$ represents the DFT total energy, $E_{H u b}$ represents term containing the Hubbard Hamiltonian to model correlated states. Here the double counting $E_{d c}$ is substructed to get the corrected term. There are two popular method to calculate $E_{d c}$, namely Arround mean-field(AMF)[65] and Fully localized limit (FLL). AMF is used to treat fluctuation of the local density in system where electron is distributed whereas the FLL method is used where the electrons are more localized in specific orbitals. My material of concern is an insulator type and hence I have used GGA functional to model the system. Self-consistency cycle for the DFT is shown in Fig 5.

\section{Minimum Energy Path (MEP)}

Finding MEP to determining Energy barrier for diffusion in case of periodic material is used vastly because of the predictive nature of the reaction path. Energy barrier is closely relating to the transition rate as per transition state theory. One of the aims of this study is to understand the $\mathrm{Li}$ diffusion and energy barrier in $\mathrm{LiPO}_{3}$ and $\mathrm{Li}_{3} \mathrm{PO}_{4}$ crystal material. To achieve the desired barrier for possible diffusion path, it is important to find the minimum energy path (MEP) for the corresponding migration process. The potential energy maximum along the MEP is the saddle point and denotes the energy barrier for the migration. There are several methods to find MEP for a particular reaction. 


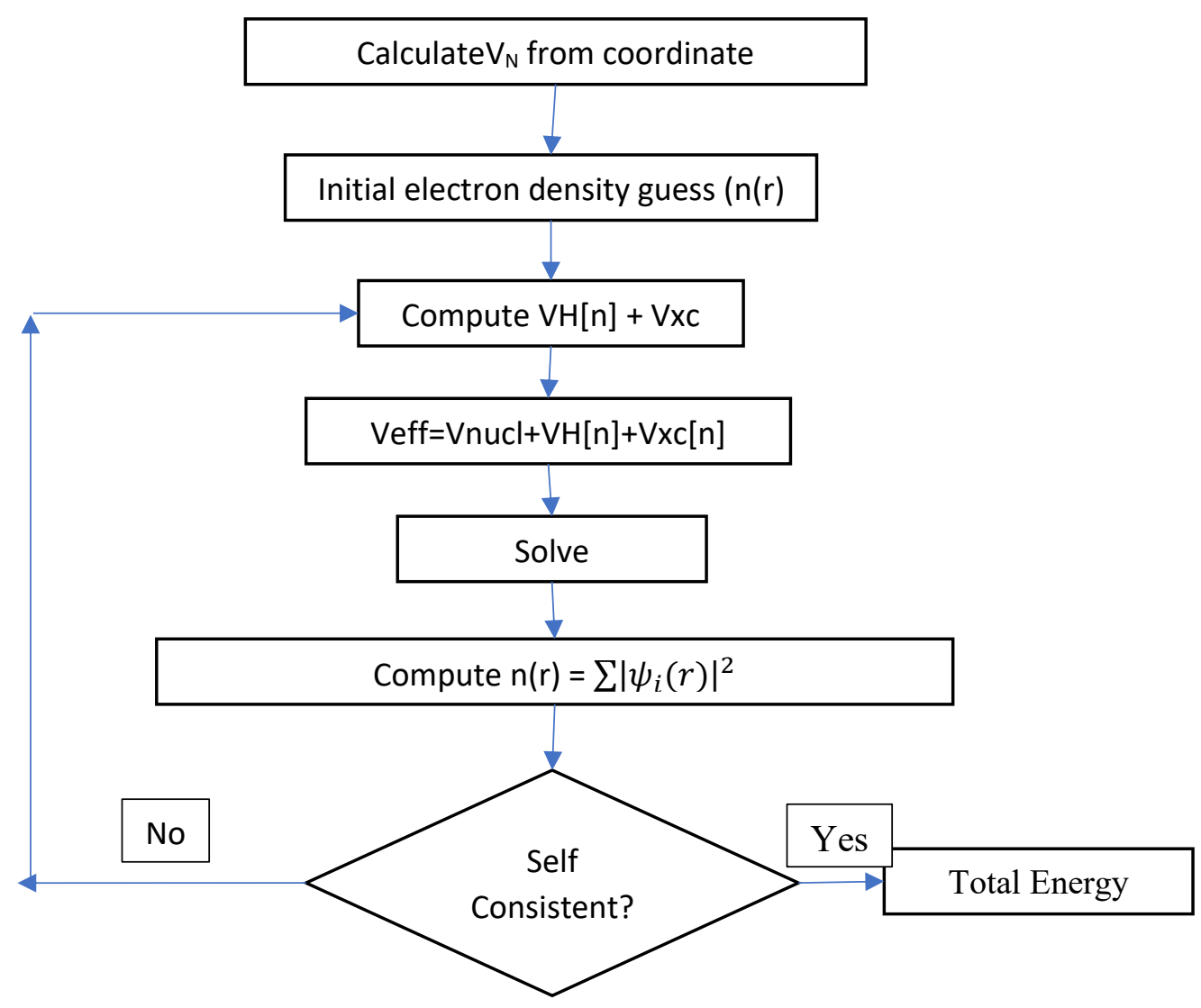

Fig 5. Energy calculation method using density functional theory

\section{Nudge Elastic Band (NEB)}

One of the effective ways to find MEP is using Nudge Elastic Band (NEB)[66] method. This method is widely used in the various system, for example, diffusion in metal surface[67], absorption of the molecule in a surface[68], metal contact formed on a surface[69] even cross slip of screw dislocation[70] involving $0.1 \mathrm{M}$ atoms in the system and $2 \mathrm{M}$ atoms in the MEP analysis. In NEB method, a string of intermediate trajectory (images) is created and placed in the path between the initial and final position of the reaction so that these images from a discrete representation of a path from the reactant (R) to the product(P). Then optimization of force is used to shift the images to the desired reaction path. In an array of path coordinates the images feel two type of force due to the 
positioning. One is a force due to the surrounding atoms and another is the spring force on each image due to other images in the reaction path. The force is represented by:

$$
F_{i}=\left.\nabla E\left(R_{i}\right)\right|_{\perp}+F_{i}^{S} \tau_{i} \tau_{i}
$$

Where $\nabla E\left(R_{i}\right)$ represents energy gradient of $\mathrm{i}^{\text {th }}$ image and $F_{i}^{S}$ is the spring force acting on image the the the I and $\tau$ is the force on each image. The perpendicular component of energy component is obtained by

$$
\left.\nabla E\left(R_{i}\right)\right|_{\perp}=\nabla E\left(R_{i}\right)-\nabla E\left(R_{i}\right) \cdot \tau_{\|} \tau_{\|}
$$

To maintain an equal spacing between images, the spring force is evaluated as:

$$
\left.F_{i}^{S}\right|_{\|}=k\left(\left|R_{i+1}-R_{i}\right|-\left|R_{i}-R_{i-1}\right|\right) \tau_{i}
$$

Where $\mathrm{R}_{\mathrm{i}}$ is the coordinate of $\mathrm{i}^{\text {th }}$ image. For the case of multiple MEP, the force optimization leads to closest MEP to the initial guess. For the case of finding the optimum between multiple MEP, global minimum on a potential energy surface is compared. Another modified version is quite frequently focusing on finding the climbing image in the MEP. CI-NEB method is a slightly modified version of NEB method. In CINEB, the image with the highest energy is identified after several relaxation steps and move uphill in energy map along the elastic band. This is done by zeroing the acting force on the image. The climbing image movement is determined by the location of the adjacent images in the path. One sample potential surface with reactant, product, and shifting of images toward the MEP is shown in Fig 6. 


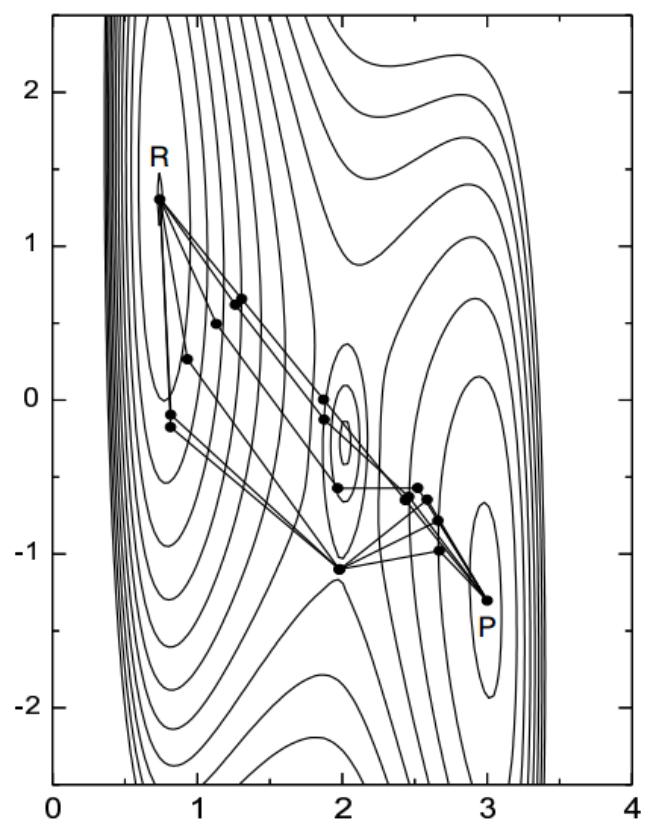

Fig 6. The Nudge Elastic Band method. CI-NEB. An elastic band is formed between the five images. The initial images are placed on the interconnected line with an equal distance. The images are then moved keeping the spring force parallel to the path and component of the true force perpendicular to the path.

\section{Ab-initio Molecular Dynamics (AIMD)}

Classical molecular dynamic (CMD) has been well established in understanding many-body condensed matter systems. In CMD the interaction between atoms is determined in advance as potentials and are divided into two-body, three-body, and many-body contributions. These interactions are approximated using different functional forms. However, as these functions are highly type of element-dependent, these make the process very selective to the material of interest and hence a myriad of interaction combination needs to be parameterized. On the other hand, interaction changes in course of the chemical process causing the normal CMD process hard to use.

$\mathrm{Ab}$ initio molecular dynamic has shown potential to solve all the discussed problems and made a bridge to the classical MD and electronic structure methods. In the 
ab initio molecular dynamics method, the energy is being calculated by the electronic structure calculations on the fly and then the force is being calculated by the derivative of the energy. Then the intermediate trajectory between two computational time-step is calculated using classical force-velocity method. In this way, it is required to calculate the electronic structure in every time step and this method is effective to approach any complex system far better than molecular dynamics diminishing dependency on the combinational empirical potential. However, in this method, new approximation dependency comes up and that is selecting approximation of solving Schrodinger equation.

Several approaches have been taken into account to incorporate a quantum approach to implementing AIMD. For example, Ehrenfest Molecular dynamics uses the time-dependent Schrodinger equation to predict particle trajectory. In Born-Oppenheimer molecular dynamics, the static electronic structure is solved for every time step for a given set of fixed nuclear positions at that instance of time. Hence it becomes a timeindependent solution of Schrodinger equation and then classical molecular dynamics approach is used to propagate the nuclei. In Born-Oppenheimer approximation the Hamiltonian is described in eqn. the total wave-function is:

$$
\phi(x, R)=\psi(x, R) \chi(R)
$$

Where $\psi$ is electronic wave-function and $\chi$ is nuclear wave-function. Nucleus wave-function is independent of electron orbitals and more localized than $\psi$. Hence Schrodinger equation can be expressed as separated from:

$$
\begin{gathered}
\left.\left[T_{e}+V_{e e}(r)+V_{e N}(r, R)\right] \psi_{n}(\chi, R)=\varepsilon_{n}(R) \psi_{n}(\chi, R)\right] \\
{\left[T_{N}+V_{N N}(R)+\varepsilon(R) \chi(R)=E \chi(R)\right.}
\end{gathered}
$$


From the Adiabatic approximation, ions move on the potential surface of the electronic ground state. So, we can rewrite the equation as:

$$
\begin{gathered}
\left.\left[T_{e}+V_{e e}(r)+V_{e N}(r, R)\right] \psi_{o}(x, R)=\varepsilon_{o}(R) \psi_{o}(x, R)\right] \\
{\left[T_{N}+V_{N N}(R)+\varepsilon(R) \chi(R, t)=i h \frac{d}{d t} \chi(R, t)\right.}
\end{gathered}
$$

The time-dependent part is replaced by classical mechanics. The resultant BornOppenheimer molecular dynamics is defined by:

$$
\begin{gathered}
M_{I} \ddot{R}_{I}(t)=-\nabla_{I} \min \left\{<\Psi_{0}\left|\mathrm{H}_{e}\right| \Psi_{0}>\right\} \\
\mathrm{E}_{0} \Psi_{0}=\mathrm{H}_{e} \Psi_{0} \\
E_{o}(R)=\varepsilon_{o}(R)+V_{N N}(R)
\end{gathered}
$$

Eo contains both contributions from the ion-ion interaction and gradient of electronic energy applicable to the electronic ground state. Where $M_{I}$ is mass of nuclei and $\mathrm{H}_{e}$ is Hamiltonian for electron. So, the nuclear equation of motion can be calculated when minimum of $<\mathrm{H}_{e}>$ is reached. To figure out the $\nabla \varepsilon_{o}(R)$ we have to use Hellmann-Feyman theorem:

$$
\begin{gathered}
\nabla \varepsilon_{o}(R)=\frac{d}{d R_{I}}\left\langle\psi_{o}\left|H_{e}(R)\right| \psi_{o}(R)\right\rangle \\
=\left\langle\nabla_{I} \psi_{o}\left|H_{e}(R)\right| \psi_{o}(R)\right\rangle+\left\langle\psi_{o}\left|\nabla_{I} H_{e}(R)\right| \psi_{o}(R)\right\rangle \\
+\left\langle\psi_{o}\left|H_{e}(R)\right| \nabla_{I} \psi_{o}(R)\right\rangle \\
=\left\langle\psi_{o}(R)\left|\nabla_{I} H_{e}(R)\right| \psi_{o}(R)\right\rangle
\end{gathered}
$$

So the force acting on an ion is the expected value of the gradient of the Hamiltonian in the ground state. Another popular approach is Car and Parinello[71]. This method relies on the rewriting of the Hamiltonian: 


$$
H_{C P}=\frac{1}{2} \sum_{i=1}^{N} m_{i} \mathbf{r}_{i}^{2}+E\left[\phi\left(\mathbf{r}_{1}, \cdots, \mathbf{r}_{N}\right)\right]+\frac{1}{2} \sum_{j} \mu \int\left|\dot{\Psi}_{j}(\mathbf{r})\right|^{2} d \mathbf{r}+L_{\text {ortho }}
$$

where $\phi\left(\mathbf{r}_{1}, \cdots, \mathbf{r}_{N}\right)$ represents the ground state electron wavefunctions. The forth term introduces kinetic energy for fictitious mass $\mu$, which represents the electronic degree of freedom. The last is to maintain the orthogonality of the system. The resulting equation of motion for Car and Parinello model then become:

$$
\left\{\begin{aligned}
\mu \ddot{\Psi}_{i}(\mathbf{r}) & =-H_{C P} \Psi_{i}(\mathbf{r})+\sum_{j} \Psi_{j}(\mathbf{r}) \Lambda_{j, i} \\
m_{i} \ddot{\mathbf{r}}_{i} & =\mathbf{F}_{i}
\end{aligned}\right.
$$

With this form, the diagonalization of Kohn-Sham matrix can be put aside in selfconsistent calculation and resulting in the same outcome. The choice between Born Oppenheimer and Car-Parrinello is case dependent and subject to a long debate. However, one noticeable[72] effort to understand the efficiency and accuracy of the two methods was performed by comparing the energy conservation of $E_{\text {cons. }}$. The conclusion was: “. . . approaches that utilize non-space-fixed bases to describe the electronic wave function, Born-Oppenheimer AIMD is the method of choice, both in terms of accuracy and speed"[72]

To transform force into velocity, a number of algorithms exist for example Verlet, Leap frog, Velo9city-corrected Verlet Algorithm etc. In Verlet algorithm, position $\mathbf{r}_{\mathrm{n}+1}$ and velocity $\mathrm{v}_{\mathrm{n}}$ can be predicted from $\mathrm{n}-1$ th and $\mathrm{nth}$ position information as:

$$
\begin{gathered}
r_{n+1}=2 r_{n}-r_{n-1+}\left(\frac{F_{n}}{m}\right) \Delta t^{2}+O \Delta t^{4} \\
v_{n}=\frac{r_{n+1}-r_{n}}{2 \Delta t}+O\left(\Delta t^{2}\right)
\end{gathered}
$$


Where, $\Delta t$ is the time step of the simulation. One thing to notice, the velocity is one step behind the real-time trajectory. All common thermal dynamic ensembles (NVE, NVT, and NPT) can be applied to AIMD simulation.

Although both the methods are quite accurate in predicting physical, electrical properties of any complex material it involves huge computational cost relative to CMD. Also, the dimension of the working sample is a big drawback of AIMD simulations. CMD can handle $10^{4}-10^{8}$ atoms whereas a general number of the atom for AIMD is $\sim 100-200$. Also, simulation time period for CMD can be reached up to several hundred of Nano-second whereas AIMD can barely hit several hundred of pico-second. Fig 7 shows a comparative simulation period and sample size data. Even with all these constraints, planned use of AIMD method can lead to outstanding findings in both crystalline and amorphous materials.

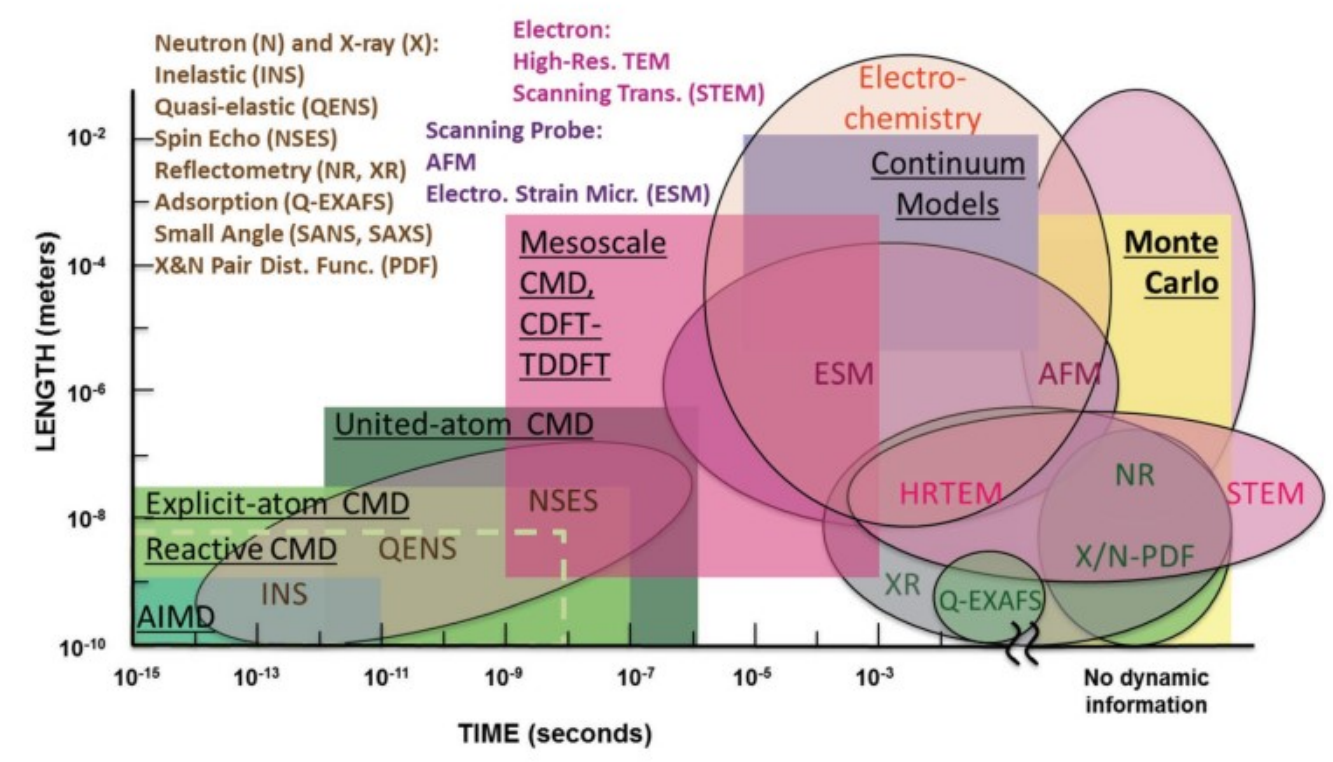

Fig 7. The length and time scales of different simulation and experimental techniques. CMD: classical molecular dynamics; AIMD: ab initio molecular dynamics; CDFT: classical density functional theory; TDDFT, time-dependent density functional theory.[73] 


\section{Pseudopotentials}

Unlike classical CMD, there is no need of interatomic potential for predicting the dynamics of an atomic structure. However, in DFT there is another approximation required to reduce the computational cost. In DFT, the calculation is based on plane wave basis set and near nuclei, the wave functions are very localized and hard to explain using plane wave. However, there is the very little contribution of the core electron (which are strongly localized in the closed inner atomic shells) to the chemical properties of the atom. Usually, the valance electron wave function realizes the core part effect in a direct way and from the orthogonality condition. Pseudopotentials is an attempt to simplify the discussed phenomena by replacing the core electrons(non-valance) and the nucleus with an effective potential so that the Schrodinger equation contains only an effective energy term instead of the Coulombic potential in the equation. The pseudopotential is such a way, the core is eliminated, and the valence electrons are described by pseudowavefunctions with fewer nodes. From the

Fig 8, we can see how a Pseudopotential acts and it shows beyond the cut-off point the effect from the pseudopotential is very same[74] as core electron orbitals. However, having a lower number of nodes makes the pseudopotential easy to calculate rather than using a large number of a plane wave to describe the oscillations in the core regions which maintain orthogonality between valance and core electrons. 


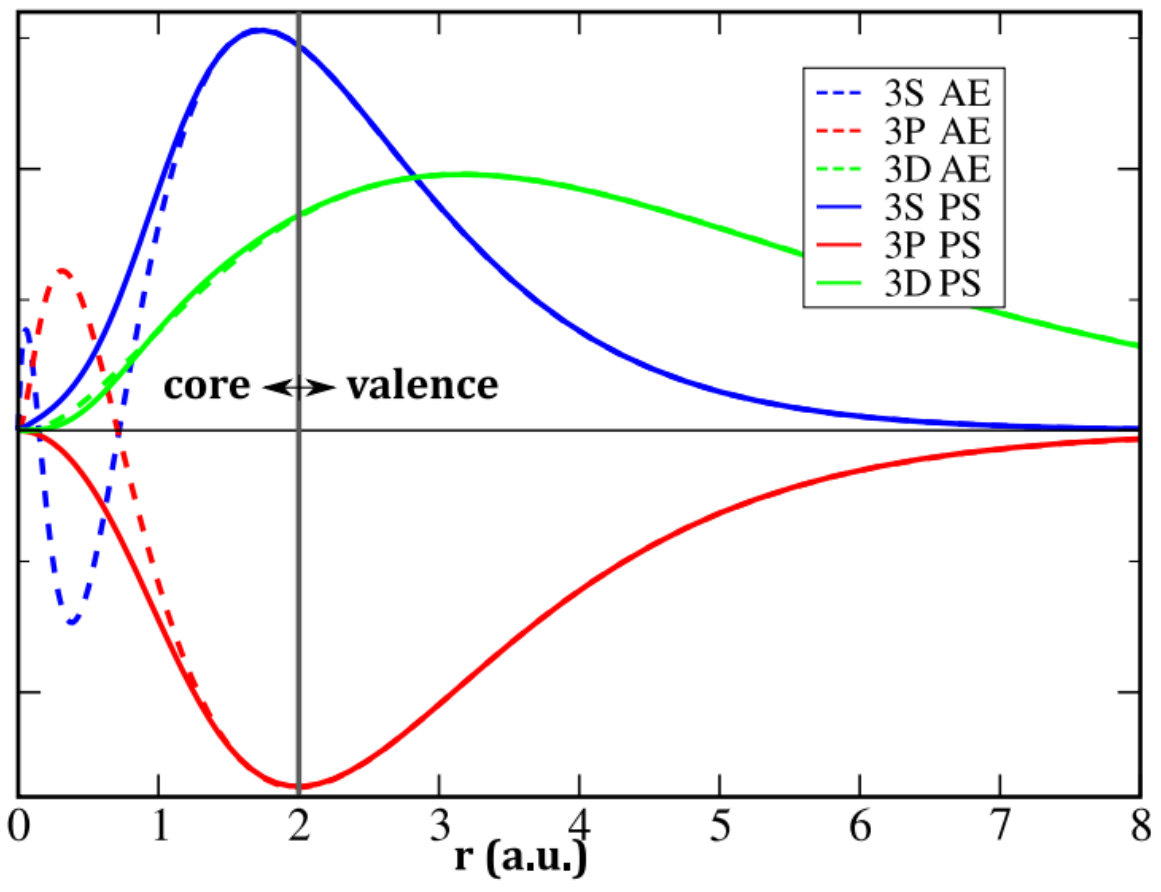

Fig 8. Schematic illustration of the pseudopotential concept. The dash lines show the allelectron wave-function, $\Psi^{\wedge} \mathrm{AE}(\mathrm{r})$ while the solid lines show the corresponding pseudowave-function, $\Psi^{\wedge} \mathrm{PS}(\mathrm{r})$ given by the pseudopotential; the cutoff radius $\mathrm{rc} \mathrm{m}$

There is a number of pseudopotentials based on their development methods like norm-conserving pseudopotentials, ultra-soft pseudopotentials, and PAW potentials.

In Norm-conserving pseudopotentials, charge within cut-off sphere are maintained fixed. Cut-off energies for first-row and transition elements is high. For the case of ultra-soft pseudopotentials (US-PP), $2 \mathrm{p}$ and $3 \mathrm{~d}$ states charge have more freedom than Norm-conservation method. It also includes the addition of charge inside the cut-off sphere to correct charge and multiple reference energies to improve charge transferability also lower cut-off energies. PAW potential is more accurate than the US-PP because of the lower radial cutoff than the radii used for the US pseudopotentials and PAW potentials reconstruct the same valance electron wave-function with all the nodes in the core region of the atoms. In this study, I have used PAW potential all way through. 


\section{Diffusivity in the Amorphous Medium}

As discussed above, a limited number of possible reaction paths makes Nudge elastic band suitable for diffusivity analysis in the crystalline structure. However, for the case of amorphous structure, lack of higher order symmetry makes thing hard or impossible to predict the possible path of ionic diffusion. Addition to that, for amorphous structure, there is no particular defined structure arrangement that makes the system random. The Hence prepared sample for each experiment will be unique in long-range order but have similar short-range ordering. All in all, there is no way to use NEB method to analyze diffusivity in an amorphous structure. There is a solution to this problem which makes it possible to calculate diffusivity from the trajectory of molecular dynamics calculation. In the following section, I will discuss the method how to derive diffusivity and conductivity from the trajectory information.

\section{Mean Square Displacement (MSD)}

In a diffusive system, a particle might follow a random walk. This is not necessarily a straight movement but might follow a zigzag path. It is trivial for a single particle to move in one direction and change in trajectory in next time span. So, to calculate the total distance the particle travels from a given position, I cannot take the vector sum of the displacement but take the absolute value of the displacement. Also, if I take the vector displacement, the total effective displacement is zero so there is no particular information to use. To consider every distance, I square the displacement and add it to previous value so that the summation is always increased and grows larger with every time step. This can give a better idea of the diffusivity of the particle. Considering 
each time step with an equal time interval, in an equilibrated system, Einstein showed that square of distance with time grows linearly with time. The process of adding a square of the displacements with time is called Mean square displacement (MSD). In molecular dynamics, it is easy to calculate the MSD from the trajectory information in an equilibrated system. The equation of MSD will be:

$$
M S D(t)=<\delta r^{2}(t)>=\frac{1}{N} \frac{1}{n} \sum_{j=0}^{n} \sum_{i=0}^{N}\left[r_{c}\left(t_{o j}+t\right)-r_{i}\left(t_{o j}\right)\right]^{2}
$$

Where $\mathrm{N}$ is the total number of a particle of interest, $\mathrm{n}$ is the number of time origin and $t_{o j}$ is the initial time at $\mathrm{j}^{\text {th }}$ step[75], [76]. One thing to note, if the time period is not long enough the MSD vs time might not necessarily follow straight line pattern. For a small time-frame when the particle does not collide with the adjacent atoms, the Newtonian velocity is linear, and the MSD is square of the displacement and hence the graph will show a parabolic trend. Over a long period of time with multiple collision, the velocity no longer is the same after the inelastic collision and hence the graph gets displaced from parabolic to straight line graph.

Albert Einstein in his thesis in 1905 showed there is a direct relationship between the diffusivity constant and MSD of the element as:

$$
\begin{aligned}
& M S D=<\Delta r(t)^{2}>\equiv A+6 D t+\text { fluctuation } \\
& D=\lim _{t \rightarrow \infty} \frac{M S D}{6 t}
\end{aligned}
$$

This is an important relationship relating macroscopic transport coefficient $\mathrm{D}$ with microscopic information on the MSD of molecular migration. This relation shows the MSD is linear to time. This is also an indicator for the equilibrated system. The one restriction is the time has to be much longer to avoid the fluctuations due to local 
vibration. In the case of molecular dynamics, the simulation time is limited to $\sim 100 \mathrm{ps}$ for AIMD $\sim 100$ ns for imperial potential based MD. So, it is important to consider the feasibility of how many particles might diffuse in the minuscular simulation timeframe. It is a common practice to increase the temperature to increase the possibility of a number of diffuse particle in the smaller time frame[52], [77]. Also for a finite system, the diffusivity will flat and drop to zero when the MSD approaches the size of the system. So, it is convenient to run the simulation up to when the MSD hit the saturation point.

In current research works, ion conductivity is quantified based on Arrhenius and Vogel-Tammann-Fulcher ion transport model. The Arrhenius model is suitable for inorganic material with a crystal structure and conductivity of the ion can be represented as:

$$
\sigma=\frac{\sigma_{o}}{T} e^{\left(\frac{-E_{A}}{K T}\right)}
$$

Where $\sigma_{\mathrm{o}}$ is constant dependent on material, $\mathrm{E}_{\mathrm{A}}$ is the activation energy, $\mathrm{T}$ is temperature, $\mathrm{K}$ is Boltzmann constant and $\mathrm{A}$ is constant. Meanwhile, Vogel-TammannFulcher model can be applied to the polymer based electrolyte. In this model, the conductivity is representing as:

$$
\sigma=A T^{-0.5} e^{\left(\frac{-B}{T-T 0}\right)}
$$

Where B is pseudo-activation energy and T0 is reference temperature.

$$
\sigma_{d c}=\frac{c_{i o n}(z e)^{2} D}{K_{B} T}
$$

Here $\mathrm{c}_{\mathrm{ion}}$ is the ion concentration, $\mathrm{z} \&$ e are the valencies of the ion and charge of the electron, $\mathrm{T}$ is absolute temperature, and $\mathrm{KB}$ is the Boltzmann constant. [2] 


\section{Density of States}

The density of the state is an important parameter to understand the vibrational characteristics of the elements in the system. From the computational perspective, the DOS is defined as:

$$
\operatorname{DOS}(n . \Delta v)=\sum \omega_{\alpha} C_{v v ; \alpha \alpha}(n . \Delta v), n=0, \ldots, N_{t}-1
$$

Where $N_{t}$ the total number of time step is, $\Delta v=\frac{1}{2 N_{t} \Delta t}$ is the frequency step, $\mathrm{n}$ is axis direction to which DOS will be calculated. $C_{v v ; \alpha \alpha}(n . \Delta v)$ is the velocity autocorrelation function. So, DOS can be calculated for user defined direction or isotropic case. The DOS can be calculated from the normalized velocity auto-correlation function (VACF) or Gaussian window can be applied in time domain to smoothen the DOS. $C_{v v ; \alpha \alpha}(n . \Delta v)$ is a very important parameter to understand the nature of force on the particles vibrating the system. This is defined as the average of the velocity and defined as:

$$
C_{v v ; \alpha \alpha}(t)=\frac{1}{3}\left\langle v_{a}\left(t_{o}\right) \cdot v_{a}\left(t_{o}+t\right)\right\rangle_{t_{o}}
$$

Where $v_{a}\left(t_{o}\right)$ is the velocity of particle " $\mathrm{a}$ " in time to. For the case of noninteracting particle, there is no change in velocity and hence the VACF is constant over time. For the case of gas a particle, the average collision distance is large and the velocity of the particle will change gradually due to collision with other particle. In this case the, velocity auto-correlation function will decay exponentially.

For the case of solids, the atomic interaction is very strong, and atom barely changes its own space. However, the atoms vibrate locally in an oscillatory motion, Because of this back and forth movement, the velocity direction also changes and makes 
the VACF be harmonic as well. However, the magnitude of the value will decay in time. So, all in all, we will have a damping harmonic shape.

In case of liquid, the interaction is less than the solid but more than gas particles. The particle has some diffusive movement and hence the oscillation mean path will increase and the VACF will also be a damping but oscillatory shape with longer decay time. The decay time will depend on the density of the system. For the case of a nonisotropic system, VACF in one direction can be defined as:

$$
C_{v v ; \alpha \alpha}(t ; n)=\left\langle v_{a}\left(t_{o} ; n\right) \cdot v_{a}\left(t_{o}+t ; n\right)\right\rangle_{t_{o}}
$$

$\mathrm{n}$ is the direction of concern and $v_{a}\left(t_{o}, n\right)$ can be defined as:

$$
v_{a}\left(t_{o} ; n\right)=n \cdot v_{a}(t)
$$

The VACF of a particle in many body systems is related to the structural factor as:

$$
G(w)=\lim _{q \rightarrow 0} \frac{w^{2}}{q^{2}} S(q, w)
$$

$G(w)$ is the Density of state. For the case of isotropic system is frequency domain:

$$
G(w)=\sum_{a} b_{a, i n c}^{2} C_{v v ; \alpha \alpha}(w)
$$

We can use Fourier transformation to calculate $C_{v v ; \alpha \alpha}(w)$ from time domain $C_{v v ; \alpha \alpha}(t ; n)$ value as:

$$
C_{v v ; \alpha \alpha}(w)=\frac{1}{2 \pi} \int_{-\infty}^{\infty} d t e^{-i \omega t} C_{v v ; \alpha \alpha}(t)
$$

For the case of non-isotropic system, the VACF will be $C_{v v ; \alpha \alpha}(t ; n)$ where $\mathrm{n}$ is the direction of concern. We have used this relation to compute the Li DOS from the AIMD trajectory information. 


\section{Bader Charge Analysis}

Bader showed how to define an atom from molecule using the charge density map[78]. He showed the topology of electron density $p(r)$ can be used to define molecule, the structure of the atom, the strength of bond as well as the stability of structure[79], [80]. A distribution of electron density in diborane is shown in Fig 9. The surface of minimum electron density perpendicular to the surface denotes the separation of atoms in the molecule. In a molecule where the electron is shared to make bond, it is very difficult to quantify the charge possession of atom. Bader theorem can be used here to segregate the charge corresponds to each atom in a molecule. The integration of charge density in the boundary calculated by Bader volume represents the charge of the particular atom[81]-[83]. This information can be used to analyze several properties of the material like an electronic moment of electron interactive molecule, the hardness of atom even energy calculation of removal of an electron from an atom. VASP or Gaussian has inbuilt code to analyze electron density file generated from the self-consistency analysis and calculate the Bader charge density of each of the element.
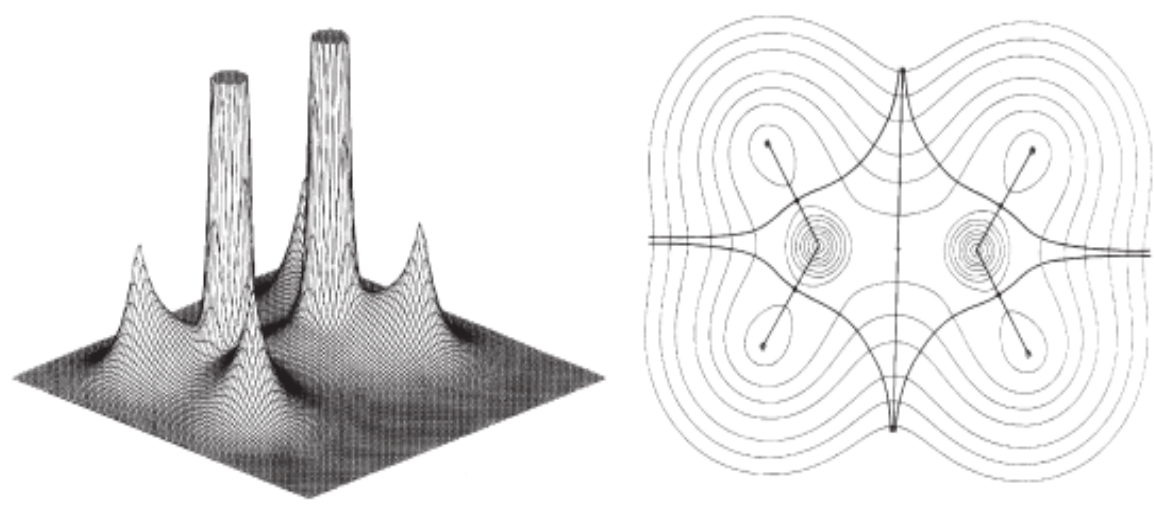

Fig 9. Electron density map of diborane in the plane of the terminal hydrogen atoms 


\section{COMPUTATIONAL DETAILS}

\section{Approach}

In order to accurately model the desired structure from experimental data and analyze Li mobility in both crystal and amorphous structure, I have chosen the approach to study very carefully. Thankfully this is not a new type of study. Several theoretical studies in an amorphous structure are already performed using classical molecular dynamics (CMD) and quantum chemistry. High level of accuracy and established method to predict diffusivity and energy barrier lead us to use electronic structure calculation. As of any experimental study, I first prepared the sample closely observing the several important parameters like lattice constant, interatomic forces, total structure energy, hoping that the sample behavior will be consistent with fundamental literature reported parameters (lattice constant, the interatomic distance, diffusivity etc.). I have considered reported experimental lattice parameter as a benchmark of the level of accuracy. In this section, I will describe the procedure to create my samples of study.

All calculations in this study are performed using atomic-scale electronic structure calculation controlled by density functional theory (DFT) approximation using projector augmented wave (PAW) method. The total work is divided into two steps: 1) diffusion analysis in crystal and 2) Ab-initio Molecular Dynamics (AIMD) calculation in the amorphous system. The first part was performed using plane wave open source package Quantum Espresso (QE) [84], while the glass part calculation is done using commercial code VASP (version 5:3:5) [85], [86]. As all of this study material is of an insulator type, The ultrasoft-type pseudopotential with Perdew Burke Ernzerhof (PBE) GGA [87]-[89] 
exchange-correlation functional is used to replace the core part of the nuclei and valence electron exchange-correlation term calculation.

\section{Li Diffusion in Crystal structure}

In [37], the dimensionality effect on Li diffusion in Li-based compounds have been thoroughly discussed. However physical and chemical stability are also critical factors to consider for the electrolyte. Having a layered structure has reportedly provided a good stability for $\gamma-\mathrm{Li}_{3} \mathrm{PO}_{4}$ structure as confirmed by several physical and computational studies [90], [91]. The $\gamma-\mathrm{Li}_{3} \mathrm{PO}_{4}$ unit cell [92] shows an orthorhombic (Pnma) space group structure. Fig 10 shows a ball-and-stick model of a $2 \times 2 \times 2$ supercells of an ideal crystal containing 2 different crystallographic Li sites based on the $\mathrm{Li}$ chain concentration $(4 \mathrm{Li}$ and $2 \mathrm{Li}$ ). The chain containing $4 \mathrm{Li}$ will be represented as "d" site and the chain contains $2 \mathrm{Li}$ will be represented as "c" site throughout the paper. In this structure, nearest neighbor of $\mathrm{Li}$ and $\mathrm{P}$ is $\mathrm{O}$.

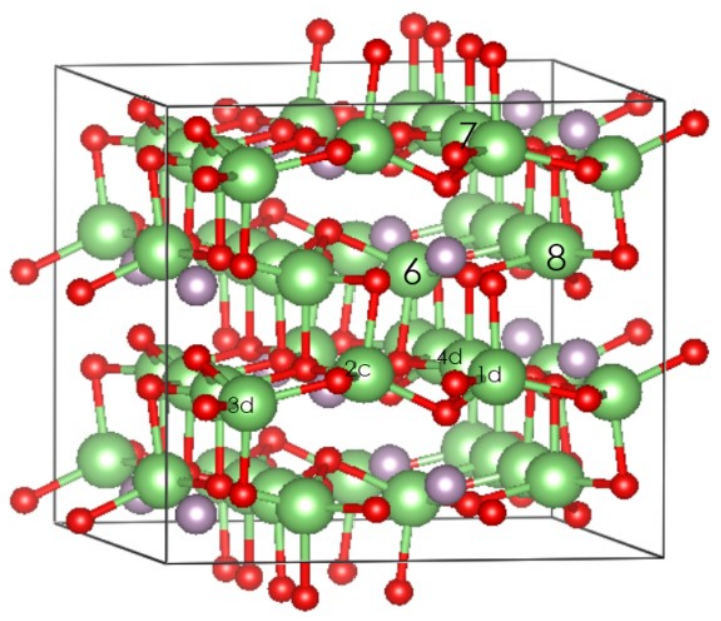

Fig 10. $2 \times 2 \times 1 \mathrm{Li}_{3} \mathrm{PO}_{4}$ supercell. Here red atoms are $\mathrm{O}$, violet indicates $\mathrm{P}$ and Green atoms are Li. 
I started with reproducing the experimental model of $\gamma-\mathrm{Li}_{3} \mathrm{PO}_{4}$ as reported several articles [37], [93], [94]. For theoretical analysis of diffusivity of an ion in a material, it is necessary to use a large structure to obtain reliable bulk diffusion properties. In my study, I have considered a periodic boundary condition. Hence it is very important to be cautious in preparing the study sample. On the other hand, in the DFT process, I cannot use a very big supercell due to the high cost related to the calculation. Hence a compromise was often made with respect to the size of the simulation box. I need to expand the supercell in such a way to keep sufficient distance between migrating ions. For LiPO3, its unit cell is already having a large area, so I didn't increase the dimension for this. After choosing the initial atomic structure, I need to process my sample using the experimentally reported lattice coordinate configurations (CIF file). As DFT is performed for calculation of $0^{0} \mathrm{~K}$ temperature, I need to optimize the structure to get the total energy minimized. To perform this task, there are several built-in codes already available in a number of ab-initio packages. One of the processes is the relaxation method where the force on each atom is calculated from the derivative of converged energy which is calculated using DFT method. Then the atomic position is adapted according to the direction of the force acting on each atom. The energy of the atomic structure is calculated estimating the electron density of the structure using plane wave method using Khon-Sham equation. The self-consistency method is discussed in DFT section. After each self-consistency cycle, the atomic position is updated from the derived force. The total process is shown in Fig 11. I need to set a cut-off value for force convergence to get the desired level of accuracy which can be estimated based on the purpose of study and material. A lower cut-off is required for high level of accuracy with much greater 
computational cost. For this method, the boundary of the structure is not changed in the whole process. In the second method, after the end of every relax method, the total boundary of the structure is also optimized for the total stress along the axis direction. This is called the volume-relax method.

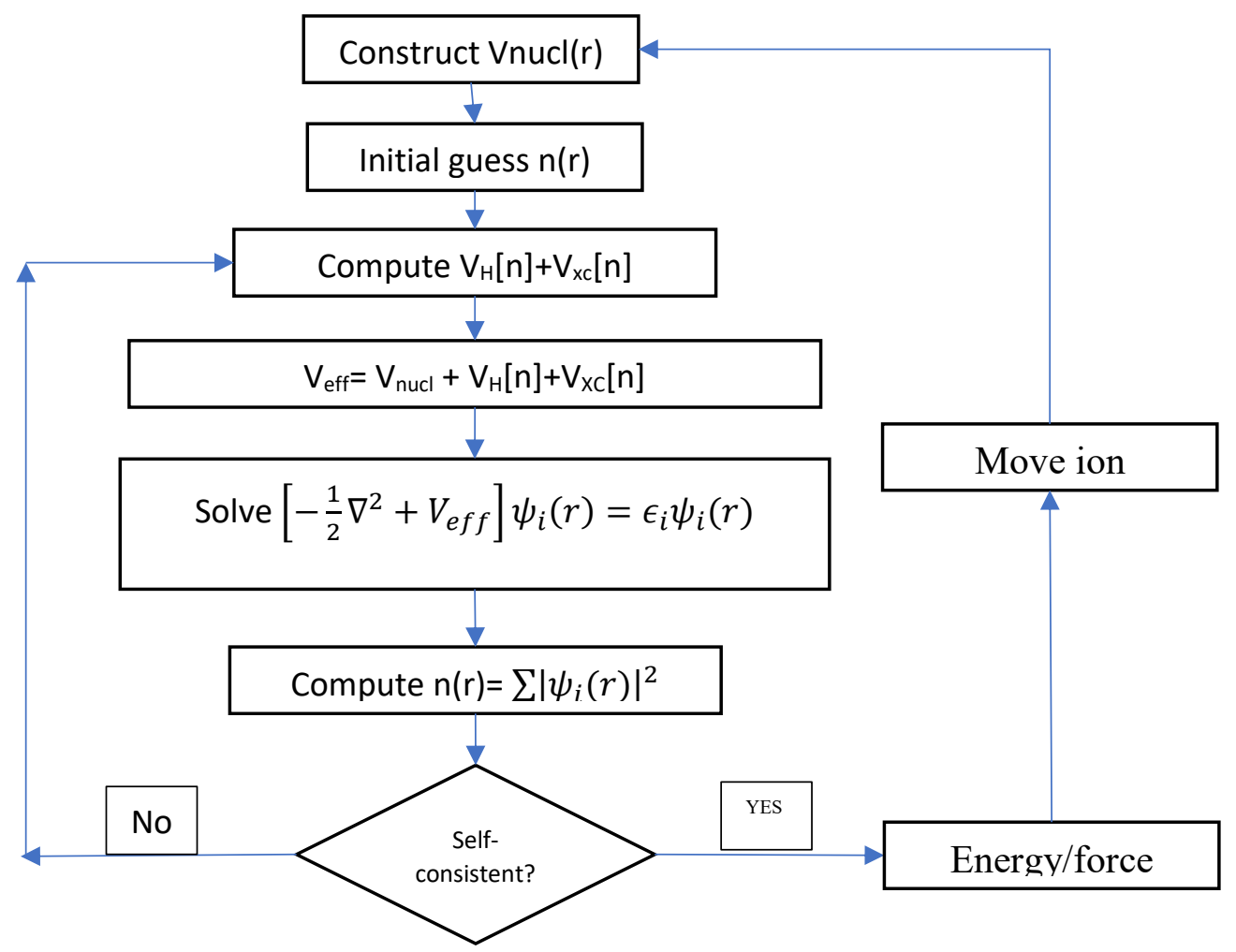

Fig 11. Relax method to get optimum energy structure

The total process will give an estimation of atomic position in the structure which is highly dependent on several parameters like force constant, energy cut-off, electron density cut-off etc. However, the pseudopotential is one of the vital factors and there are several of them available for each of the element. The pseudopotentials are classified based on several aspects like method of exchange functional used (PBE, PW, BLYP, PBESOL, PERDEW-ZUNGER LDA, GGA), type of the pseudopotentials (PAW, 
ULTRASOFT, NORMCONS etc.). Even though several different types of pseudopotential can be mixed with taking extra caution but it is trivial to use the same type of function for each element maintaining the same parameter as discussed above. An open source software package like Quantum Espresso doesn't have any controlled process to maintain the accuracy of the available pseudopotentials and their development process also aimed for a different objective. Some of the pseudopotentials are great to calculate the energy of the system but fails for relaxing the structure in $0^{\circ} \mathrm{K}$ and vice versa. So, this is important to choose the right pseudopotential for my purpose and test it beforehand. For my case, I need to first estimate the new atomic coordinate as the environment of the structure changes. I have checked several available potentials for $\mathrm{Li}$, $\mathrm{O} \& \mathrm{P}$ and performed the volume relaxation method to achieve the similar lattice constant as reported from the experimental analysis. I have found PBE type Vanderbilt ultra-soft based potential works very well with my sample Li3PO4. After choosing the pseudopotential, the next step is to choose the right energy cut-off as discussed in DFT section. From the Fig 4, I can estimate $\mathrm{h} 2 / 2 \mathrm{~m}|\mathrm{~K}+\mathrm{G}|^{2}=30$ Ry is reasonably good for the calculation. Next, I relax the structure in $0^{\circ} \mathrm{K}$ using volume relax method embedded in Quantum espresso so that the maximum total ionic force is lower than $10^{-4} \mathrm{Ry} /{ }^{\circ} \mathrm{A}$. The calculated structural parameters are listed with experimental data in Table 3Error! Reference source not found.. The script used for calculation is available in the Appendix.

Due to its higher Li concentration, its existence in both crystal and glass phases and the presence of a long 1-D chain of $\mathrm{PO}_{4}$ arrangement, $\mathrm{LiPO}_{3}$ has attracted a great research attention for Li diffusion study [2], [95]. The standard experimental structure 
[96] for $\mathrm{LiPO}_{3}$ was used in this study to evaluate the Li migration characteristics in the crystalline structure, where a long chain of Li is surrounded by PO4 tetrahedral such as shown in Fig 12.

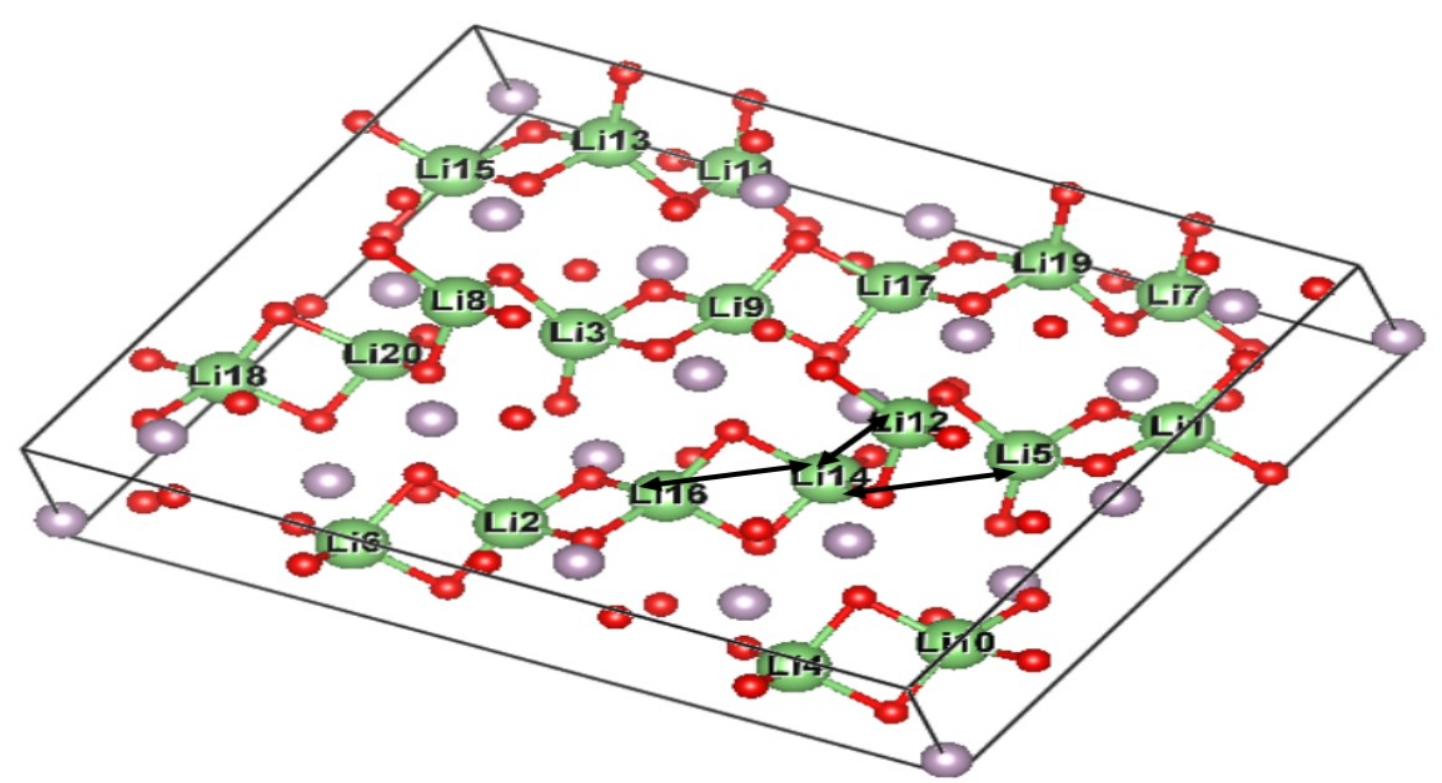

Fig 12. $\mathrm{LiPO}_{3}$ supercell showing chain of $\mathrm{Li}$ and the arrows shows the sites of interest for diffusion calculation

Table 3. Lattice constants for $\gamma-\mathrm{Li}_{3} \mathrm{PO}_{4}$

\begin{tabular}{|c|c|c|c|c|}
\hline & $\operatorname{Ref}[37]$ & $\operatorname{Ref[93]}$ & $\operatorname{Ref}[94]$ & This work \\
\hline $\mathrm{a}\left({ }^{\circ} A\right)$ & 10.58 & 10.53 & 10.49 & 10.52 \\
\hline $\mathrm{b}\left({ }^{\circ} A\right)$ & 6.17 & 6.12 & 6.12 & 6.1378 \\
\hline $\mathrm{c}\left({ }^{\circ} A\right)$ & 4.99 & 4.93 & 4.9266 & 4.953 \\
\hline
\end{tabular}

The $\mathrm{PO}_{4}$ tetrahedral makes a tunnel where $\mathrm{Li}$ is surrounded with the oxygens attracted by the electrostatic force. To proceed with the simulation, the initial structure is relaxed using the volume relaxation method also so that the maximum total ionic force is lower than $10-4 \mathrm{Ry} /{ }^{\circ} \mathrm{A}$. The integration over the Brillouin zone is carried out with $2 \times 2$ 
$\times 2$ Monkhorst-Pack grids for geometry optimization of the supercell. The relaxed structure shows a relatively small $3: 5 \%$ increase in volume compared to the one reported in an experimental study [97] which is shown in Table 4.

Table 4. Lattice constants for $\mathrm{LiPO}_{3}$

\begin{tabular}{|c|c|c|}
\hline & $\operatorname{Ref}[38],[96]$ & This work \\
\hline $\mathrm{a}\left({ }^{\circ} A\right)$ & 16.45 & 17.033 \\
\hline $\mathrm{b}\left({ }^{\circ} A\right)$ & 5.405 & 5.56353 \\
\hline $\mathrm{c}\left({ }^{\circ} A\right)$ & 13.806 & 13.43987 \\
\hline
\end{tabular}

Using both of the previously described structures, QE in-built Nudge elastic band (NEB) [84] method is used for investigating the energetics of several probable diffusion processes. In NEB, it is assumed [98] that the diffusion is slow enough to follow Boltzmann statistics and the process consists of transition states which pass through harmonic regions of the potential energy surface minima and saddle points. As no special image is predicted in the diffusion process, relaxed first and last image coordinate is given to the code and combined with 5 intermediate steps so that I can capture the whole trajectory. Quasi-Newton Broyden's method is used for image optimization. The interchain and intra-chain vacancy diffusion energetic profiles are shown in Fig 13 and Fig 14. From the figures, it can be concluded that the nearest neighbor of $\mathrm{Li}$ in the images are oxygen. Li migration caused these bonds to break or be created. Even though throughout the diffusion process surrounding atoms move for optimum energy structure, the $\mathrm{Li}-\mathrm{O}$ interaction energy plays the most vital role in the energetics of Li movement. In the NEB process, the energy profile of intra-chain Li migration energy profile (1d-2c-3d on Fig 10) and interchain (1d-4d on Fig 10) Li movement energy is investigated. Moving from 
$1 \mathrm{~d}$ to $2 \mathrm{c}$, the first image shows Li formed a tetrahedral with 1,2,3,4 oxygen, when moving to the second image, one bond with oxygen loosens up causing increased energy. In the consecutive image, however, the bond breaks down. In the fourth image, the surrounding condition remains the same but by the fifth image, the loose 3rd O bond breaks down and the Li bonds with 5th oxygen causing a drop in total energy whereas, in the last image, the Li reaches the minimum energy condition by making a tetrahedral. A similar mechanism can be found in the migration of Li from $2 \mathrm{c}$ to the $3 \mathrm{~d}$ site. For the case of $1 \mathrm{~d}$ to $2 \mathrm{c}$ migration, the activation barrier is much lower $(0.346 \mathrm{eV})$ because only one effective Li-O bond was broken in the process, in contrast with the $2 \mathrm{c}-3 \mathrm{~d}$ migration, where $2 \mathrm{Li}-\mathrm{O}$ bonds break down in the time when $\mathrm{Li}$ reaches the saddle point causing a much higher activation energy $(0.69 \mathrm{eV})$.
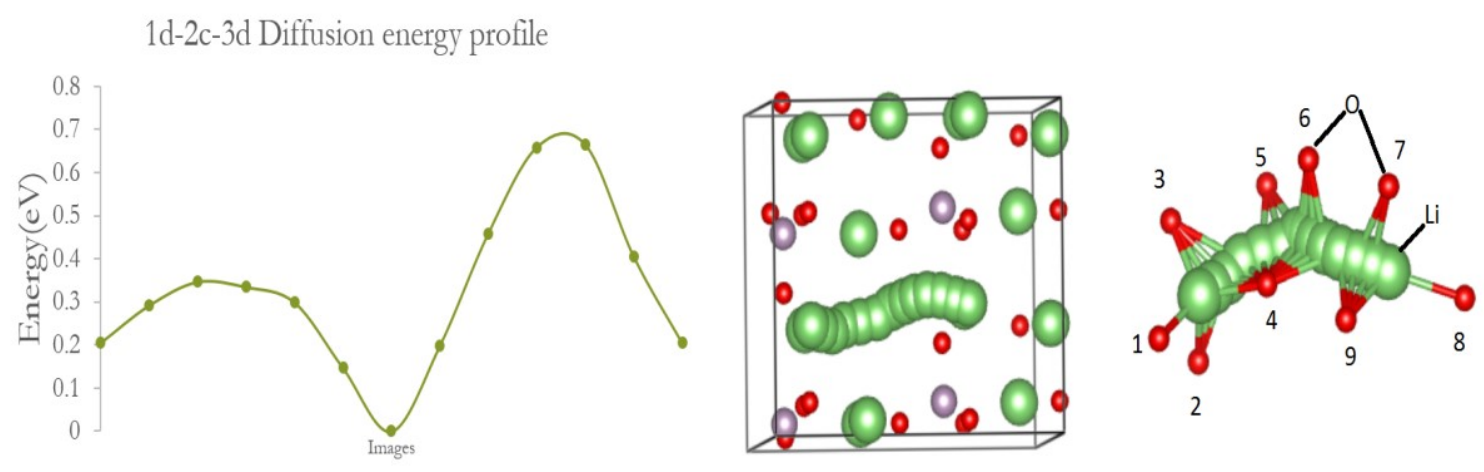

Fig 13. Li-ion migration a) total energy profile of supercell in $1 \mathrm{~d}-2 \mathrm{c}-3 \mathrm{~d}$ site migration $\mathrm{b}$ ) cross-section of the migration process $\mathrm{c}$ ) Interaction of $\mathrm{Li}$ with oxygen in the migration process in a $\mathrm{Li}_{3} \mathrm{PO}_{4}$ supercell. Green indicates $\mathrm{Li}$, Red indicates $\mathrm{O}$ atom. 

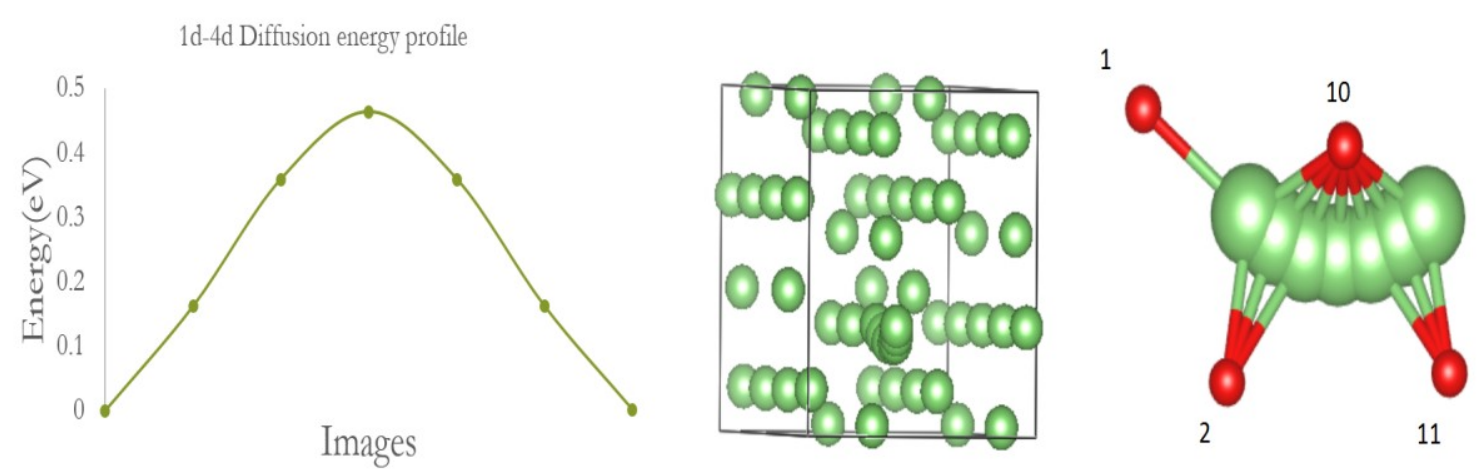

Fig 14. Li-ion migration a) total energy profile of supercell in 1d-4d site diffusion b) crosssection of the migration process $\mathrm{c}$ ) Interaction of $\mathrm{Li}$ with oxygen in migration time in $\mathrm{Li}_{3} \mathrm{PO}_{4}$ supercell

On the other hand, Fig 14 shows a $0.4998 \mathrm{eV}$ energy barrier as this process consists of only breaking $1 \mathrm{Li}-\mathrm{O}$ bond. These activation energies provide a qualitative idea of possible Li migration trajectory in Li3PO4 material. All the calculated migration paths are shown in Fig 15. In [25], Xingfeng et. al. showed a curve path is possible for Li diffusion and my achieved paths also followed the same trend. For phosphate type material, simulation output [47] shows a wavy diffusion path and proved experimentally[99]. The curve nature comes from the associated element that helps the ion to jump. For example, Fig 13 c, \#1 oxygen is associated with the Li-ion migration process and the curvature of the Li-ion is centering the \#1 oxygen. For the case of Fig 14.c, we can see the same trend is applicable. However, for Fig 16.c, the 2-associated oxygen is swinging the Li to guide the ion to follow an arc path. In Phosphate type material Oxygen always accompany the journey and hence carve will have one or multiple bends in its path. Fig 15 shows an aggregated coordinate of all the atoms in the NEB images and their energy profile. We can see from Fig 15 b that the "c" site does possess lower energy than the "d" site Li. This is due to the size effect of $\mathrm{P}$ compared to Li atom. In both "c" \& "d" sites, there are 4 anions packed in the same 
length of space where "c" site contains four Li-ion and "d" site contains $2 \mathrm{Li}$ and $2 \mathrm{P}$ atom. Hence it is obvious the packing concentration inside "d" is higher than the "c" site which increases the energy as well. Also, we can notice the wavy pattern of the arrangement of Li-P-Li-P due to the relaxation process whereas Li chain in site "d" is almost a straight line.
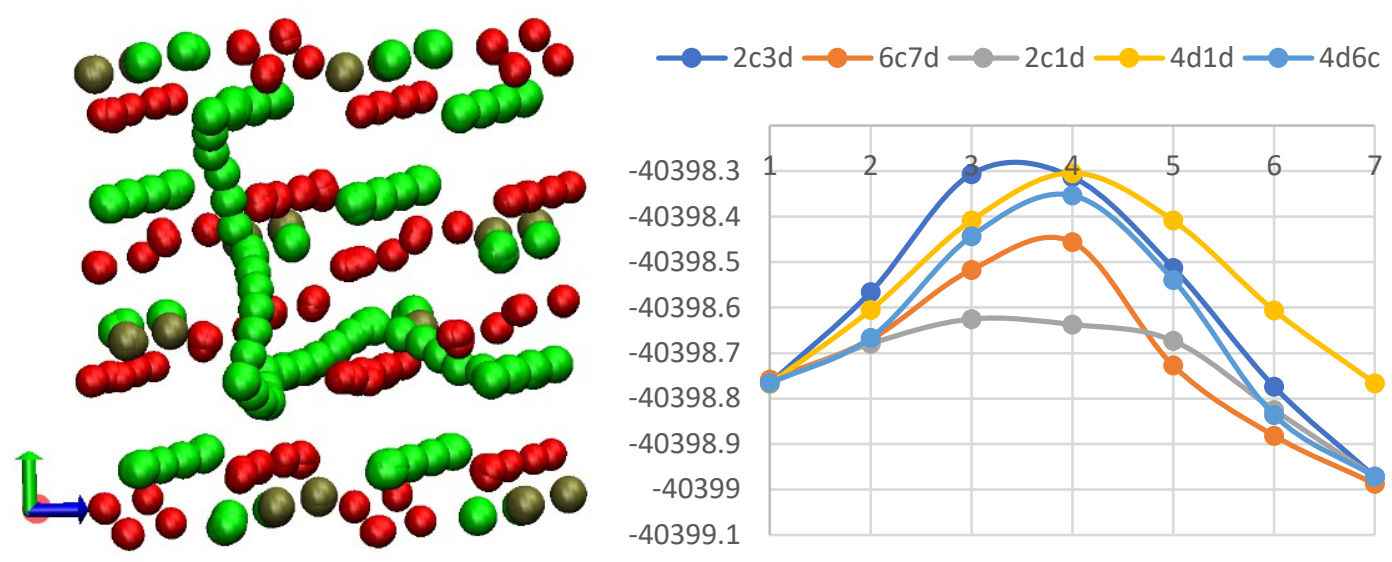

Fig 15. a. Possible Li diffusion paths in Li3PO4 crystal supercell. b. absolute energy profile for all of them c site in left, $d$ in right.
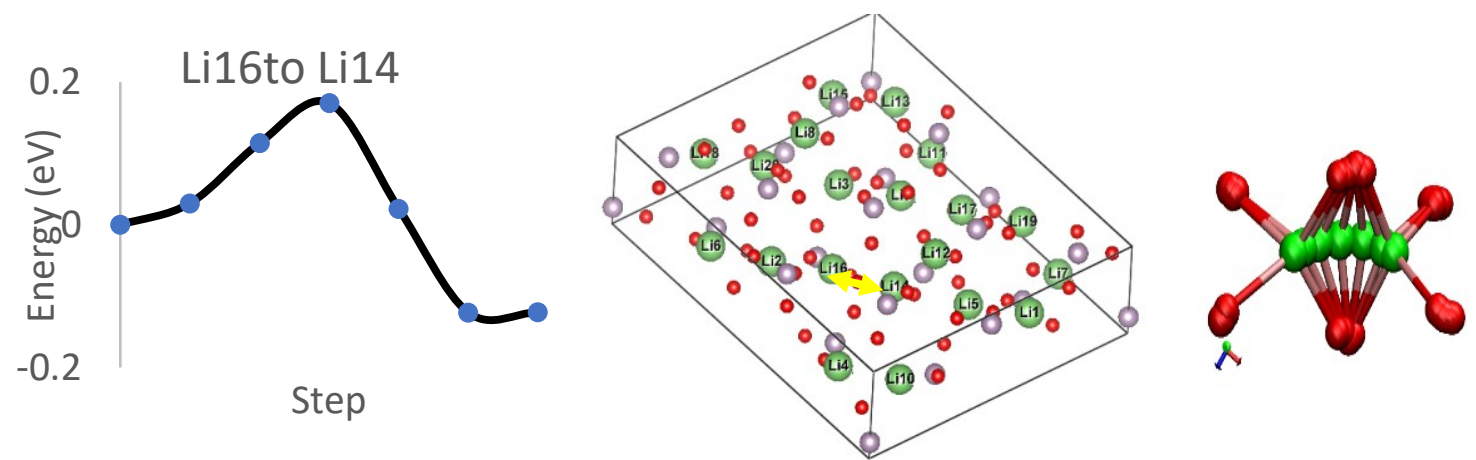

Fig 16. Li-ion migration a) total energy profile of supercell in Li16-Li14 site diffusion b) top view of the migration process c) aggregated snapshots of the migration process showing the interaction of $\mathrm{Li}$ with oxygen in the interim process in $\mathrm{LiPO}_{3}$ supercell.

Aside from the bond analysis to understand the migration barrier, I have tried to understand if the charge distribution is related to the migration barrier as well. To do so, Bader charge analysis (check Appendix) is employed to understand the ion transfer effect 
in term of the change in charge. Bader analysis is performed for each of the images which provide the effective charge of each element and also provides the atomic volume. A typical charge distribution data is shown in Fig 17. We can observe the charge for $\mathrm{Li}$ is almost constant in the whole process and some charge of $\mathrm{O}$ and $\mathrm{P}$ does change. We can see for each of $\mathrm{P}$ or $\mathrm{O}$ element, there are 2 bands of charge in the structure.

I am also interested to understand why there is a change of charge and what the effect of this charge is over the total energy profile of the migration. A comparison of all calculated diffusion path along with the previously reported data is shown in Table 5. The diffusion study shows a minimum of activation energy on $\mathrm{LiPO}_{3}$ consisting of a long chain of Li as shown in Fig 12. Noticeably Li12 occupy out of chain position. So, we investigate 3 migration energetics for this structure namely, Li16 to Li14, Li14 to Li12, Li14 to Li15. For LiPO3, the Li16-Li14 shows a $0.2936 \mathrm{eV}$ barrier and Fig 16 show the trajectory and corresponding energy profile. From the breaking of the Li-O ionic bond as of above discussion, I can conclude that the surrounding $\mathrm{O}$ atmosphere is controlling the Li trajectory and activation energy. In [100], Marc Enger et.al reported the stable $<\mathrm{Li}-\mathrm{O}>$ bond length for Li tetrahedral are 1.96 and Octahedral Li showed a $<\mathrm{Li}-\mathrm{O}>=2.15 \mathrm{~A}$. They got distorted by strong oxygen-oxygen edge distortion. From the bond length analysis, for site 14, we can find the initial Li from a tetrahedral and Li-O bond lengths are 1.88, 1.86, 2.11 and $2.18 \mathrm{~A}$ with an average of $<\mathrm{Li}-\mathrm{O}>=2.0075 \mathrm{~A}$ which shows a large deviation from the normal estimated Li-O interaction. For the case of site 16, also we can see the bond lengths are $2.09,2.10,1.89 \& 1.88 \mathrm{~A}$ with an average of $<\mathrm{Li}-\mathrm{O}>=1.99 \mathrm{~A}$ and hence also a deviation from the standard statistical data. A closer $<$ Li-O $>$ toward stable 1.96 corresponds to the stable configuration at " 16 " site. These bond length data give a 
hint of higher energy for the Li sites and loose bonding of Li-O for the case of Li-O $>2$

A. These explain why the energy at saddle point is still low compared to the initial states even when the Li has a coordination number of 2 in the saddle point. To understand the effect, several points to notice. First, for the case of Li around the vacancy defect, the LiO bonds that face towards the vacancy, will be less than the opposite site as Li-ion will tend to displace to the vacancy due to an imbalance of force. This causes the nearest neighbor and some of the next nearest neighbor to displace from the initial position. In Fig 18 , the picture represents a $\mathrm{Li}_{3} \mathrm{PO}_{4}$ structure with vacancy and the center of the $\mathrm{Li}$ tetrahedral are the vacant $\mathrm{Li}$. The larger atoms represent having a significant force due to the vacancy of the Li. We can clearly see the nearest neighbor is having force due to this displacement.

Table 5: Comparison of Energy barrier in different path in different crystal structure \& comparison with reported work

\begin{tabular}{|c|c|c|c|}
\hline Crystal Formula & Diffusion Path & $E_{b}$ Reported $[37](e V)$ & $\mathrm{E}_{\mathrm{b}}$ This work $(\mathrm{eV})$ \\
\hline \multirow{5}{*}{ 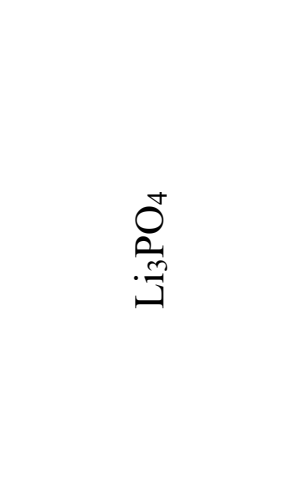 } & $2 c-1 d$ & 0.36 & 0.36 \\
\hline & $4 d-1 d$ & 0.45 & 0.463 \\
\hline & $4 d-6 c$ & 0.55 & 0.5309 \\
\hline & $7 d-6 c$ & 0.63 & 0.618331 \\
\hline & $2 c-3 d$ & 0.69 & 0.67 \\
\hline \multirow{2}{*}{ 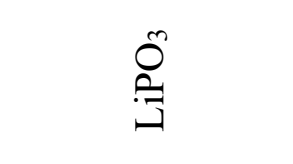 } & Li16-Li14 & - & 0.294 \\
\hline & Li14 to $\mathrm{Li} 12$ & - & 0.31 \\
\hline
\end{tabular}


Change in charge in 2C-1d NEB path

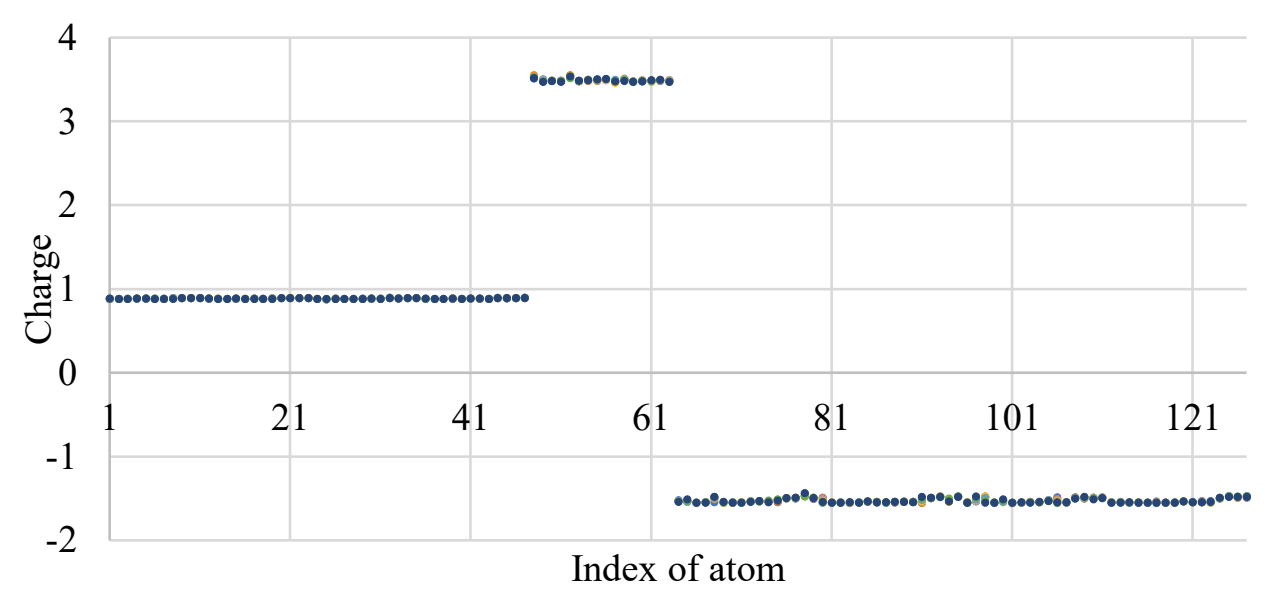

Fig 17. Aggregated change in charge in all the images for $2 \mathrm{c}-1 \mathrm{~d}$ diffusion

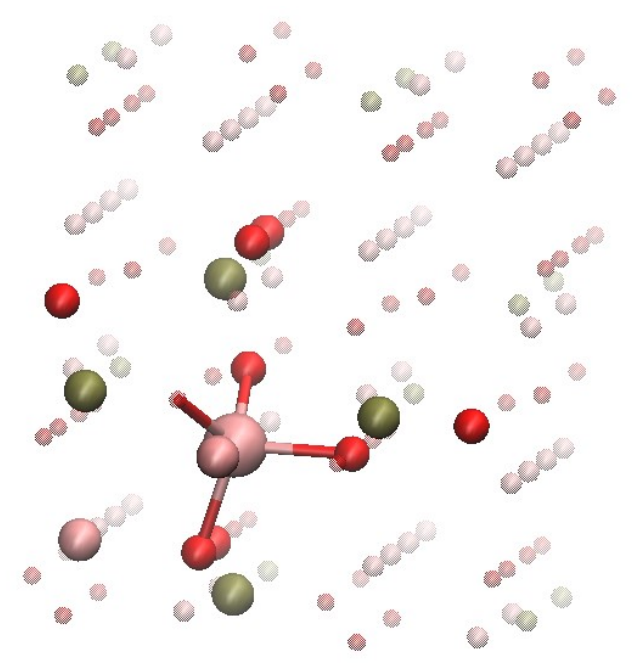

Fig 18. Center of the tetrahedral Li(cyan, larger atom) indicate vacancy site. The surrounding highlighted atoms are experiencing a significant force due to the vacancy defect of Li.

I have investigated the effect of compression and expression on the diffusion in several possible diffusion paths in $\mathrm{Li}_{3} \mathrm{PO}_{4}$ structure to understand the behavioral characteristics of Li diffusion and to understand and design a better structure for fast diffusion. The idea is to understand if expanding or compressing the structure give a 
similar effect to the Li migration path and how does the energy barrier change. To examine this, I choose 3 different paths ( $4 \mathrm{~d} 1 \mathrm{~d}, 2 \mathrm{c} 1 \mathrm{~d} \& 2 \mathrm{c} 3 \mathrm{~d})$ and have expanded and compressed the structure. The resulting P-O bonds are more resistive to the force than $\mathrm{Li}$ O bonds. From the Fig 19, we can see that the bond length of P-O and Li-O changes linearly with volume and the slope suggest that P-O shows a lower sensitivity to the volume change of the total structure. Along the same line, it can be surmised that the effect of volume change is only felt by the Li-O bond. A volumetric compression of $4.4 \%, 8.7 \%, 14.3 \% \& 27.1 \%$ are performed while the structure is also elongated up to $4.6,9.3 \& 15.8 \%$.

The energy profile for each path is shown in Fig 20. It can be seen, the barrier increased with the expansion of the structure for $4 \mathrm{~d} 1 \mathrm{~d}$ and $2 \mathrm{c} 3 \mathrm{~d}$ path. For example, the barrier for $2 \mathrm{c} 3 \mathrm{~d}$ increases from 0.66 to $0.69 \mathrm{eV}$ and decreased to $0.42 \mathrm{eV}$ at $9.3 \%$ volume compression. Particularly interesting is the very deep barrier decrease in the extreme $15.8 \%$ compression. On the other hand, exactly the opposite outcome can be seen as the least energy barrier path in the normal $2 \mathrm{c} 1 \mathrm{~d}$ migration. It can then be concluded that the energy barrier does not necessarily follow the structure reformation. This observation can be used to decrease the energy barrier for high barrier paths and find an optimum condition for fast Li diffusion in SSE.

In [101], Smekens et.al showed an increase in performance by using higher density Li crystal structure. They concluded that higher density electrode shows a higher discharge rate at low current rates whereas, for high current density, low-density electrodes show better performance. However, as can be seen from Fig 21, the energy of each image of each path always increases with both expansion and compression. A linear change in 
volume changes the average bond length between the elements which leads to increase the total energy of the system. This is demonstrated in Fig 22 \& Fig 24, where the change in energy for an image of a path is plotted against the change of volume. The plotted points resemble a curve that can be fitted with a $3^{\text {rd }}$ order polynomial. Thus, the minimum energy corresponding to the optimum lattice constant of the structure can be achieved with volume relaxation method. Any change in volume either expansion or compression gives rise to increase in energy. However, from Fig 21, I can see there is a slight change in energy for each of the image maintaining the same volume. In particular, observe that a position change of traveling $\mathrm{Li}$ has a noticeable change in the $\mathrm{Li}-\mathrm{O}$ bond in the images while other atoms do not experience any significant change.

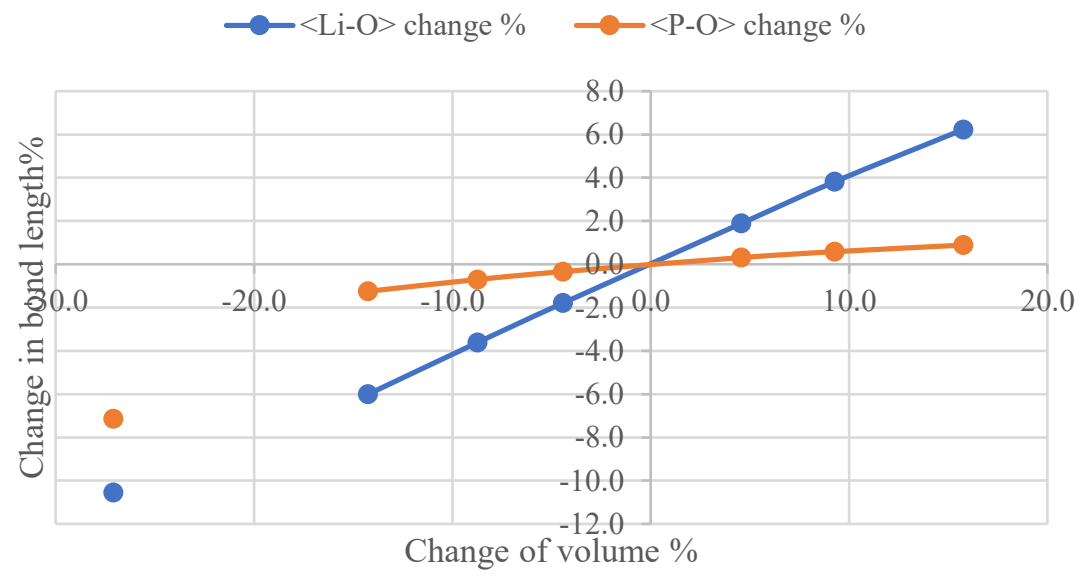

Fig 19. Change of Li-O and P-O bond with respect to the total volume change

One example of this phenomenon can be seen in Fig 19, where the average bond lengths for different images of all $\mathrm{Li}$ and $\mathrm{P}$ atoms in the structure of the $4 \mathrm{~d} 1 \mathrm{~d}$ path are plotted. Some big deflection is visible for atom 3, whereas the changes are smaller for Li $10 \& \mathrm{Li} 34$. These Li $10 \& \mathrm{Li} 34$ atoms are near to the migration path, which adjusts its 
position with respect to the change in vacancy due to the migration of atom 3 . As we have already seen the fact that between P-O \& Li-O bond length, $\mathrm{Li}-\mathrm{O}$ is more sensitive to volume change, I can assume the contribution of $\mathrm{P}-\mathrm{O}$ bond to the energy is practically negligible, while Li-O bond length has the largest effect. Fig 22 shows that the total energy can be fitted to a $3^{\text {rd }}$ order polynomial of Li-O bond length. This has a significant importance in understanding how the Li-O barrier changes with the change in volume.

Let's consider one particular case and discuss qualitatively how we can understand the energy barrier trend on volume change for a particular path. Fig 23 suggests that Li-O bond lengths are almost stable for all of the Li atoms except the migrating $\mathrm{Li}$.

Barrier at different lattice constant for path 2c3d

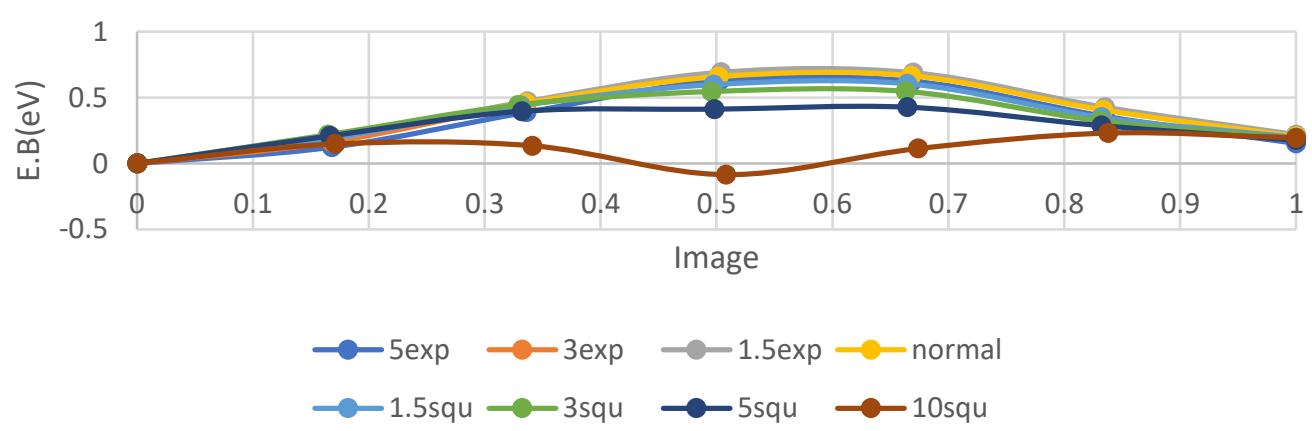

Barrier at different lattice constant for path $2 \mathrm{c} 1 \mathrm{~d}$

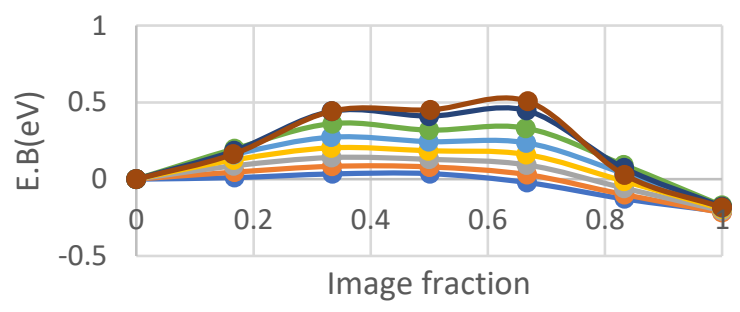

$\multimap-3 \exp \multimap-1.5 \exp -$ normal $-1.5 \mathrm{squ}$ $\multimap 3$ squ $\multimap-5$ squ $\multimap 8$ squ $\multimap 10$ squ
Barrier at different lattice constant for path $4 \mathrm{~d} 1 \mathrm{~d}$
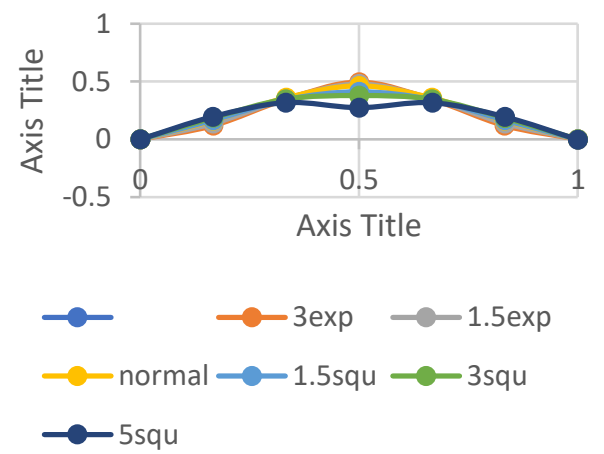

Fig 20. Energy barrier for different paths 1. 2c1d 2. 1d4d 3. 2c3d 
Therefore, it is reasonable to assume that the resulting polynomial is a linear combination of similar $\mathrm{E}(<\mathrm{Li}-\mathrm{O}>$ ) for each $\mathrm{Li}$ atom. Even though $\mathrm{Li}-\mathrm{O}$ bond length differs from "d" \& "c" sites, each Li-O energy level will follow the similarly reduced polynomial. By taking derivative of the polynomial fit, I can find the bond length of minimum energy is $1.99557 \AA$. Now, consider Li migration in the $4 \mathrm{~d} 1 \mathrm{~d}$ path with $4.56 \%$ expansion compared to the relaxed structure. For the $1^{\text {st }}$ image, the $<\mathrm{Li}-\mathrm{O}>$ is $2.047514 \AA$ and for the transition state (TS) image the $<\mathrm{Li}-\mathrm{O}>$ is $2.125831 \AA$ with a reduction of coordination number $(\mathrm{CN})$ by 1 . This reduction of nearest neighbor's distance is applicable for all the compression and expansion in the 4d1d path TS' migrating Li's CN. Thus, the TS is already having a higher energy than the initial state (IS) by one Li-O equivalent energy.
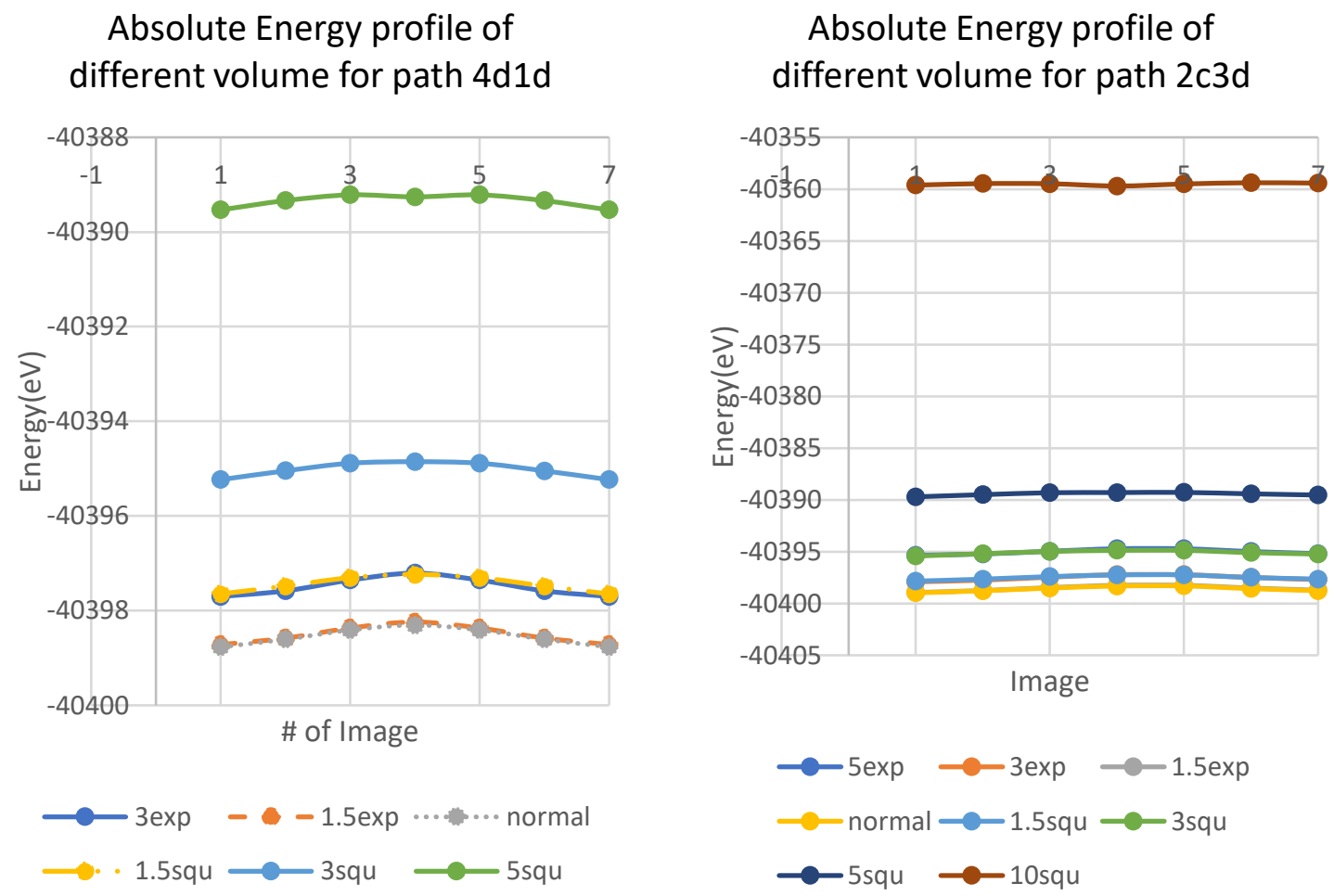

Fig 21. Energy profile of $4 \mathrm{~d} 1 \mathrm{~d}$ and $2 \mathrm{c} 3 \mathrm{~d}$ paths in different structural volume 


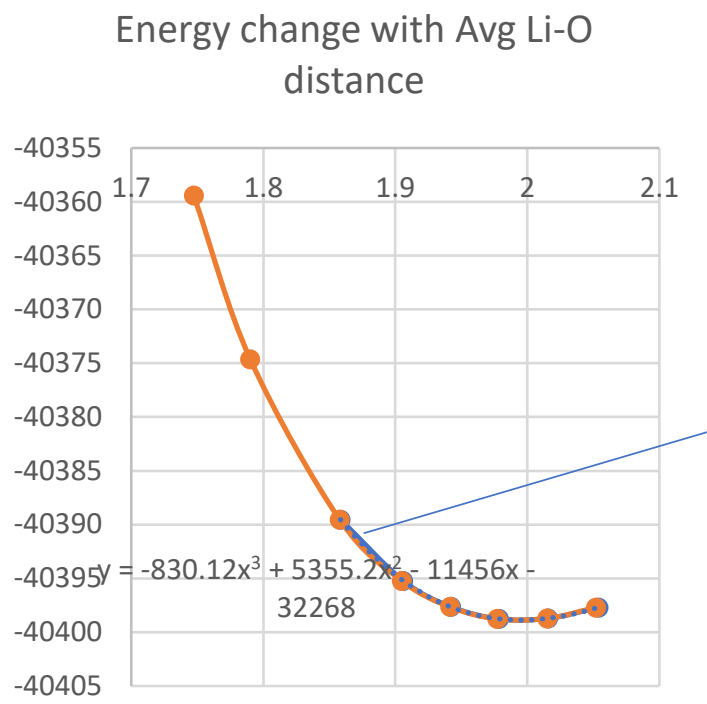

\section{Energy change with Avg Li-O \\ distance for $2 \mathrm{c} 1 \mathrm{~d}$}

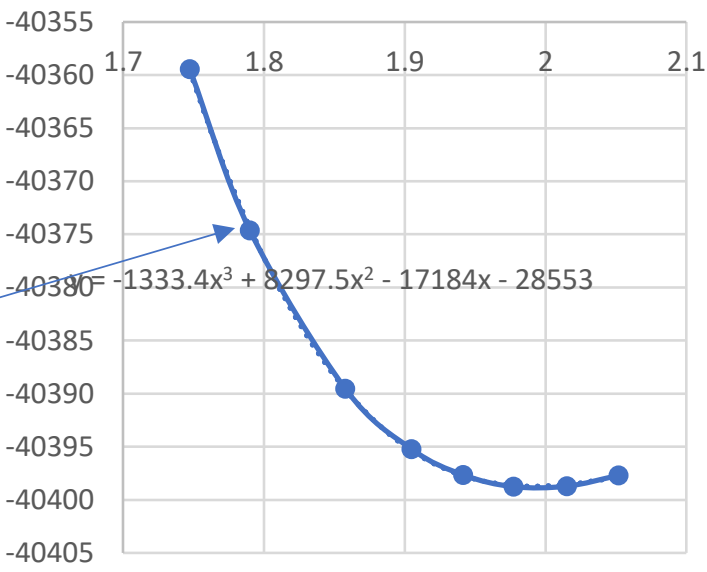

Fig 22. Energy vs $<\mathrm{Li}-\mathrm{O}>$ bond length relation for $4 \mathrm{~d} 1 \mathrm{~d} \&$ 2c1d path

On the other hand, for the $9.3 \%$ volume expansion from the Table 7 , it can be seen that the energy increase coincides with the average increase of the Li-O bond of migrating Li in the TS state. From Table 7, a linear fraction 'average' of (-40395.51+ $40399.261)=3.751 / \mathrm{n} \mathrm{eV}$ (where $\mathrm{n}$ is a positive number) is used to obtain the $\mathrm{Li}-\mathrm{O}$ bond break energy. Similarly, for the case of $4.56 \%$ expansion, the $<\mathrm{Li}-\mathrm{O}>$ change effect has 3.726/n eV energy, which is lower than the previous case. Added to the Li-O bond breaking energy, this change will yield a lower barrier than the level for $9.3 \%$ volume change. Similarly, I have calculated the same for the other cases of volume change. The result shows that the energy barrier will decrease with compression which matches with the NEB calculation result of the energy barrier for different volume change. 


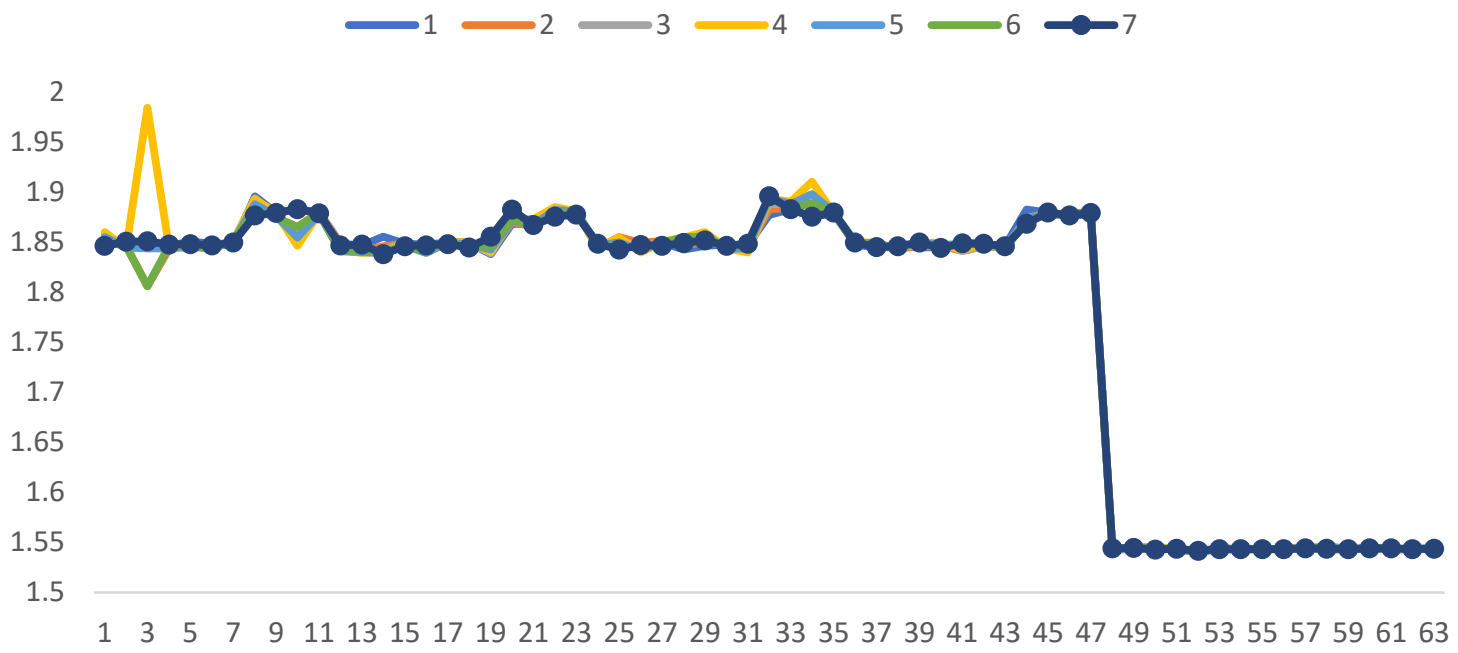

Fig 23. Change in $\mathrm{O}$ bond distance for each $\mathrm{Li}$ and $\mathrm{P}$ in Li3PO4 structure

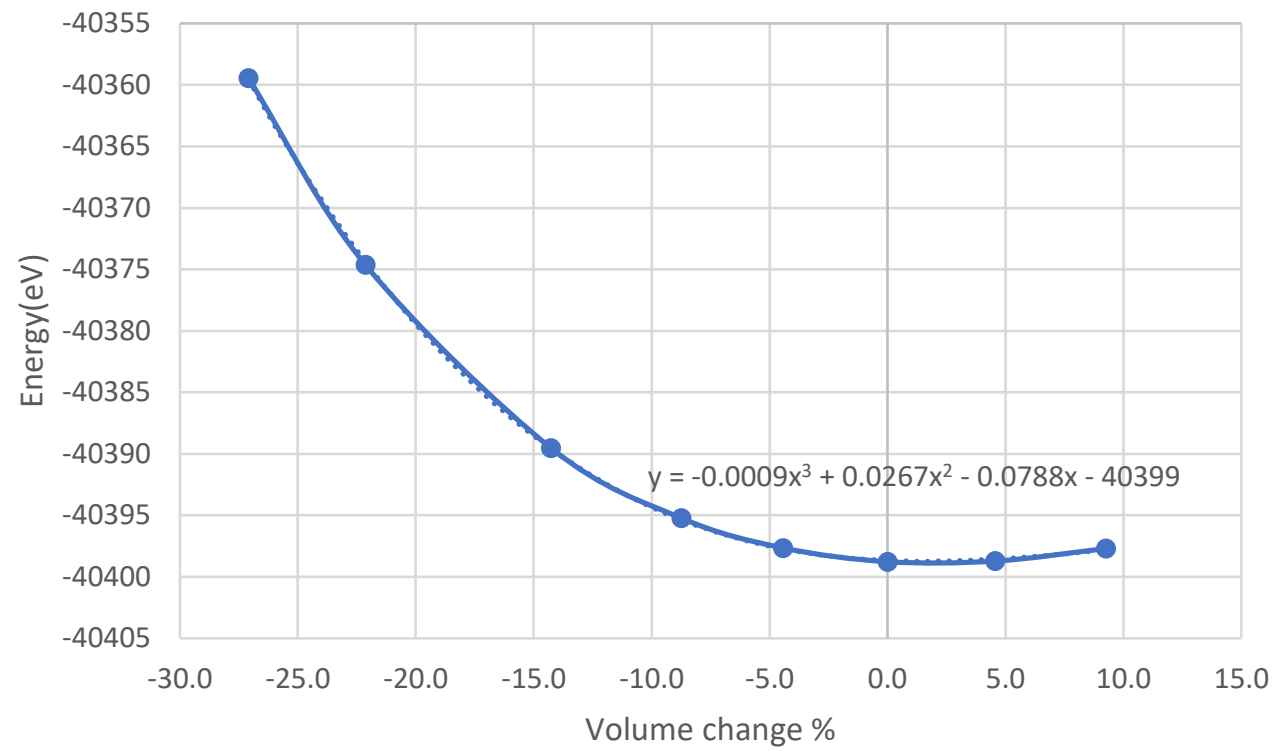

Fig 24. Energy vs volume for path 4d1d

Now for the case of $2 \mathrm{cld}$, the opposite relation seems to hold. I also try to estimate the trend using the same process. I find for the $2 \mathrm{c} 1 \mathrm{~d}$, the polynomial has similar roots of $1.9917 \AA$ with matching form, as can be seen in Fig 22 a. For this case, the $\mathrm{CN}$ also drops from 4 to 3 at TS. Thus, effectively one Li-O bond is severed. At the same time, from 
Table 8 , it can be seen that the energy for changing Li-O distance increases with compression which adds to the bond energy of $\mathrm{Li}-\mathrm{O}$ and hence energy increases for the 2c1d site. To summarize, from this discussion I can conclude several important findings:

1. The energy and distance of $\left\langle\mathrm{Li}-\mathrm{O}>\right.$ in $\mathrm{Li}_{3} \mathrm{PO}_{4}$ follow a $3^{\text {rd }}$ order polynomial curve.

2. The relative $<\mathrm{Li}-\mathrm{O}>$ bond position between TS and IS is an important aspect in determining the energy curve. They will determine if the energy increases or decreases with respect to the change of volume.

3. Change of $\mathrm{CN}$ is important to understand the energy barrier magnitude. A large difference of $\mathrm{CN}$ will lead to higher EB as some large bond breaking energy needs to be overcome

Table 6. Comparison of bond length over different volume

\begin{tabular}{|c|c|c|c|c|c|c|c|c|c|c|c|}
\hline & \multicolumn{4}{|l|}{$2 \mathrm{c} 3 \mathrm{~d}$} & \multicolumn{4}{|l|}{ 4d1d } & \multicolumn{3}{|l|}{ 2c1d } \\
\hline $\begin{array}{l}\text { vol } \\
\text { ch } \\
\text { an } \\
\text { ge }\end{array}$ & $\begin{array}{l}\mathrm{Li}- \\
\mathrm{O}(\% \\
\text { chan } \\
\text { ge) }\end{array}$ & $\begin{array}{l}\text { P- } \\
\text { O( } \\
\% \text { ch } \\
\text { ang } \\
\text { e) }\end{array}$ & $\begin{array}{l}\text { TS } \\
\text { Li-O } \\
\text { (\%ch } \\
\text { ange) }\end{array}$ & $\begin{array}{l}\text { TS } \\
\text { Li-O } \\
\text { (\%ch } \\
\text { ange) }\end{array}$ & $\begin{array}{l}\mathrm{Li}- \\
\mathrm{O}(\% \\
\text { chan } \\
\text { ge) }\end{array}$ & $\begin{array}{l}\text { P- } \\
\text { O( } \\
\% \text { ch } \\
\text { ang } \\
\text { e) }\end{array}$ & $\begin{array}{l}\text { TS } \\
\text { Li-O } \\
\text { (\%ch } \\
\text { ange) }\end{array}$ & $\begin{array}{l}\text { TS } \\
\text { Li-O } \\
\text { (\%ch } \\
\text { ange) }\end{array}$ & $\begin{array}{l}\text { Li- } \\
\mathrm{O}(\% \\
\text { chan } \\
\text { ge) }\end{array}$ & $\begin{array}{l}\text { TS } \\
\text { Li-O } \\
\text { (\%ch } \\
\text { ange) }\end{array}$ & $\begin{array}{l}\text { TS } \\
\text { Li-O } \\
\text { (\%ch } \\
\text { ange) }\end{array}$ \\
\hline $\begin{array}{r}27 . \\
1\end{array}$ & 10.5 & -7.1 & -11.3 & -13.8 & & & & & 10.5 & -11.9 & -7.4 \\
\hline & & & & & & & & & & -9.8 & -6.6 \\
\hline $\begin{array}{r}- \\
14 . \\
\end{array}$ & -6.0 & -1.2 & -5.8 & -2.0 & -6.0 & -1.3 & -6.1 & -5.1 & -6.0 & -6.4 & -4.9 \\
\hline $8 . \overline{7}$ & -3.6 & -0.7 & -3.2 & 3.5 & -3.6 & -0.7 & -3.7 & -2.4 & -3.6 & -4.0 & -3.3 \\
\hline $4 . \overline{4}$ & -1.8 & -0.3 & -1.7 & 0.7 & -1.8 & -0.3 & -1.9 & -0.9 & -1.8 & -2.1 & -1.6 \\
\hline 4.6 & 1.9 & 0.3 & 2.0 & 0.9 & 1.9 & 0.3 & 1.9 & 0.9 & 1.9 & 1.4 & 1.7 \\
\hline 9.3 & 3.8 & 0.6 & 3.9 & 1.8 & 3.8 & 0.6 & 3.8 & 1.6 & 3.8 & 0.4 & 3.5 \\
\hline $\begin{array}{r}15 . \\
8\end{array}$ & 6.2 & 0.9 & 6.6 & 1.9 & & & & & & & \\
\hline
\end{tabular}


Table 7. $4 \mathrm{~d} 1 \mathrm{~d}<\mathrm{LiO}>$ distance, $\mathrm{CN}$ of migrating Li with structure energy for each image

\begin{tabular}{|l|l|l|l|l|l|l|}
\hline $\begin{array}{l}\text { Volume } \\
\text { change(\%) }\end{array}$ & 9.3 & 4.6 & 0 & -4.4 & -8.7 & -14.3 \\
\hline TS $<$ Li-O $>$ & 2.125831 & 2.110097 & 2.091636 & 2.073821 & 2.042078 & 1.984884 \\
\hline IS $<$ Li-O $>$ & 2.047514 & 2.009558 & 1.972395 & 1.93428 & 1.898719 & 1.851412 \\
\hline $\begin{array}{l}\text { Energy at } \\
\text { TS }\end{array}$ & -40395.5 & -40396.4 & -40397.4 & -40398.2 & -40399.4 & -40400.1 \\
\hline $\begin{array}{l}\text { Energy at } \\
\text { IS }\end{array}$ & -40399.3 & -40400.1 & -40399.9 & -40398.5 & -40395.8 & -40389.6 \\
\hline $\mathrm{dE} / \mathrm{n}$ & 3.751 & 3.726 & 2.563 & 0.315 & -3.596 & -10.513 \\
\hline
\end{tabular}

Table $8.2 \mathrm{c} 1 \mathrm{~d}<\mathrm{LiO}>$ distance, $\mathrm{CN}$ of migrating $\mathrm{Li}$ with structure energy for each image

\begin{tabular}{|l|l|l|l|l|l|l|l|}
\hline $\begin{array}{l}\text { Volume } \\
\text { change } \\
(\%)\end{array}$ & 9.3 & 4.6 & 0 & -4.4 & -8.7 & -14.3 & -22.1 \\
\hline TS & 2.007117 & 1.972489 & 1.93909 & 1.907237 & 1.87531 & 1.843553 & 1.749 \\
\hline IS & 1.95618 & 2.016081 & 1.974404 & 1.933186 & 1.896657 & 1.848141 & 1.779 \\
\hline $\begin{array}{l}\text { Energy } \\
\text { at IS }\end{array}$ & -40398.1 & -40398.1 & -40397.1 & -40395.1 & -40391.6 & -40386.6 & \\
\hline $\begin{array}{l}\text { Energy } \\
\text { at TS }\end{array}$ & -40397.7 & -40398 & -40398.1 & -40396.8 & -40394.1 & -40387.5 & \\
\hline dE/n & -0.40324 & -0.04534 & 1.002928 & 1.76196 & 2.448547 & 0.832548 & 10.84 \\
\hline
\end{tabular}

First, the Li-O bond towards the vacancy will be less than the opposite site as Liion will tend to displace the vacancy due to an imbalance of force. Secondly, we can discuss a sample ionic bond characteristic. In Fig 25, a sample ionic interatomic pair potential is shown. The potential energy $\mathrm{E}(\mathrm{r})$ where $\mathrm{r}<\mathrm{ro}$, increases very fast and for the case of $r>$ ro, the potential energy change is not that significant. Now, for 16-14 path, in the end point of 14 , the bond length of 2.11 and 2.18 is getting increased to $~ 3.4 \mathrm{~A}$ and the bond length of $1.86 \& 1.88$ reduced to 1.80 and $1.82 \mathrm{~A}$ with a coordination number of 2, the change in energy is significantly lower in compared to $\mathrm{Li}_{3} \mathrm{PO}_{4}$ paths, where the $\mathrm{Li}$ $\mathrm{O}$ bond lengths are closer to $\mathrm{r}_{\mathrm{o}}$ and changes in exponential part of the carve. For example, 
for $4 \mathrm{~d} 6 \mathrm{c}$ diffusion the bond length of $2.02,2.01,1.93,1.92$ changes to $3.16,2.76,1.77 \&$ 1.76. As the changes are near to the ro(1.96 A), the change is much higher than $\mathrm{LiPO}_{3}$ paths. However, this situation of higher $<\mathrm{Li}-\mathrm{O}>$ is another cause of instability of $\mathrm{LiPO}_{3}$ with the electrolyte interface. Yifei $\mathrm{Mo}[102]$ et al showed that $\mathrm{LiPO}_{3}$ reaction energy with $\mathrm{Li}_{3} \mathrm{PS}_{4}$, LLZO, LCO L0.5CO are -32, -201, -76, -19 meV per atom where $\mathrm{Li}_{3} \mathrm{PO}_{4}$ are $0,0,0,0$ respectively. Also, $\mathrm{Li}_{3} \mathrm{PO}_{4}$ is also stable with $\mathrm{Li}_{3} \mathrm{P}$ and $\mathrm{Li}_{2} \mathrm{O}$ cathode[103]. This situation suggests that to compare the energy barrier in between multiple materials, we must take account of the initial state not just the intermediate state of the migration paths.

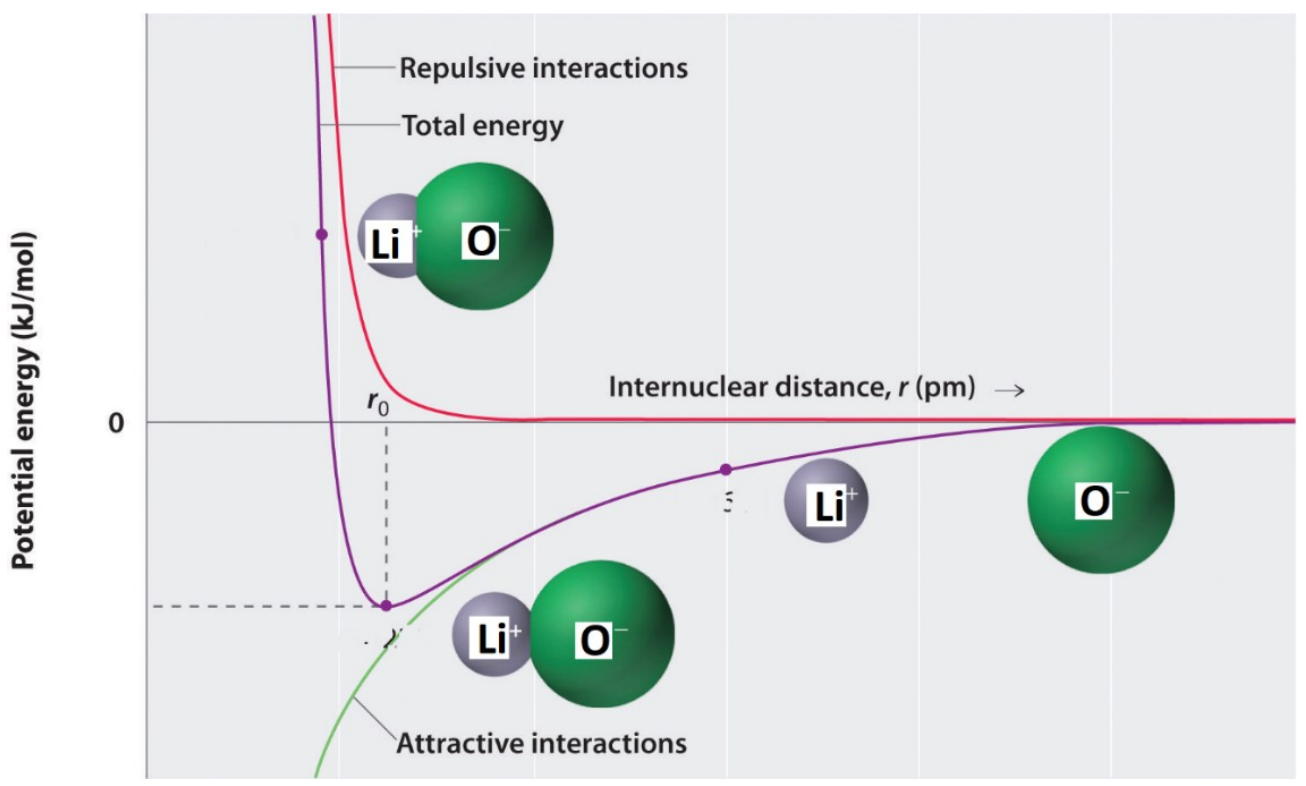

Fig 25. A sample Ionic bond energy vs inter-atomic distance plot

Fig 16 \& Fig 26 show the energy profile for Li5-Li14 and Li 14 to Li12 migration. We notice here that Li5 to Li14 movement shows a stable point in the diffusion process whenever we see no Li occupancy from the x-ray diffraction data. 
Because the activation energy is as low as $0.094 \mathrm{eV}$, there is a possibility for $\mathrm{Li}$ to overcome the vibration energy at even at room temperature for $\mathrm{Li}$ and cause no occupancy in the site. From these activation energy data, we can conclude, $\mathrm{LiPO}_{3}$ offers much smoother $\mathrm{Li}$ diffusion $\left(0.0648 \mathrm{eV} /{ }^{\circ} \mathrm{A}\right)$ than $\mathrm{Li}_{3} \mathrm{PO}_{4}\left(0.1688 \mathrm{eV} /{ }^{\circ} \mathrm{A}\right)$ crystal. However, Li14- Li12 shows endpoint is not a very stable configuration and there is an equivalent stable point in the migration path. This is a strange outcome.
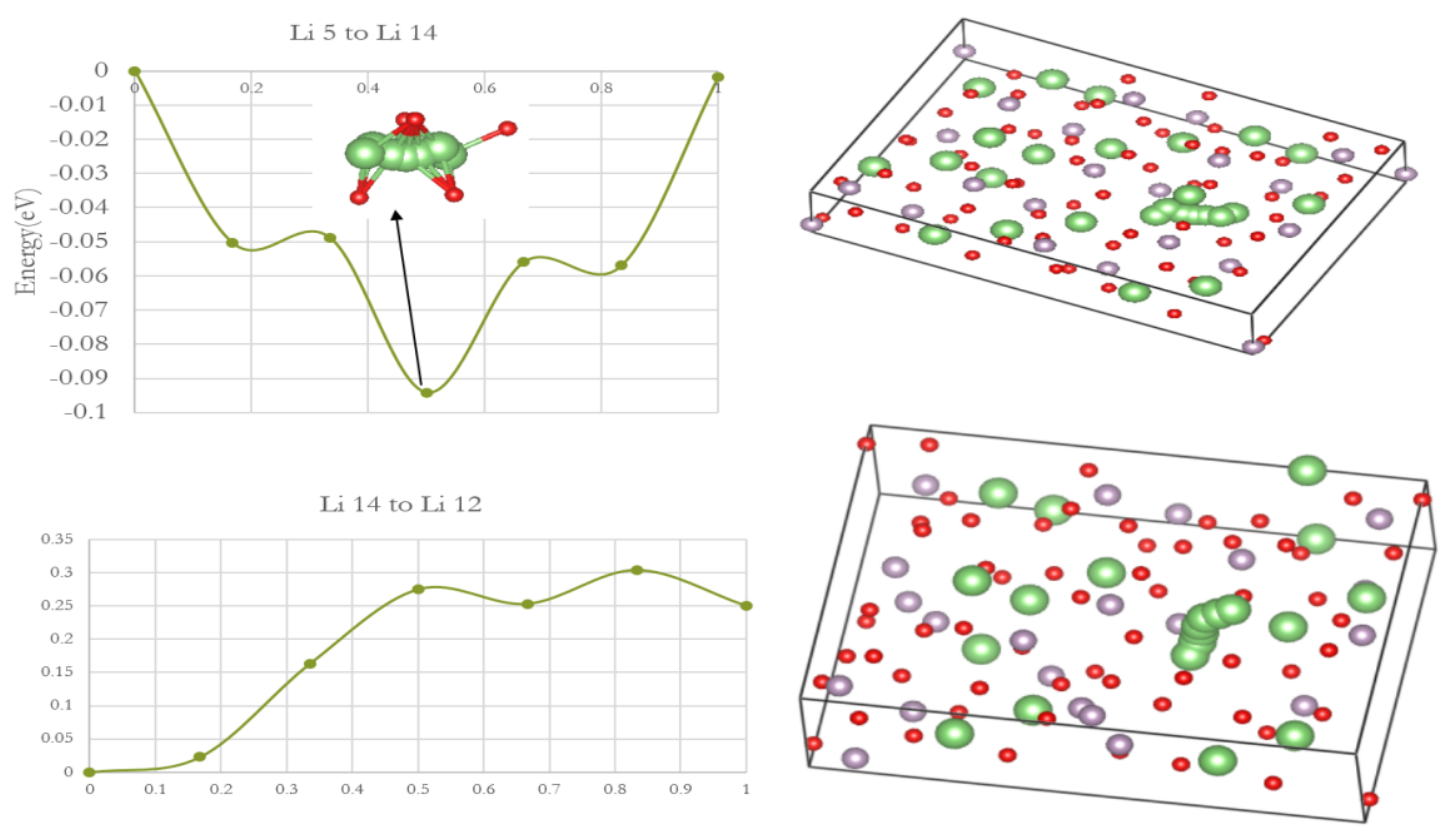

Fig 26. Li-ion migration a) energy profile in Li4-Li12 site migration b) top view of the migration process c) energy profile in Li14-Li12 site migration d) top view of the migration process in $\mathrm{LiPO}_{3}$ supercell

\section{Li Diffusion in Glass Structure}

According to Zachariasen rule, some oxide (network former) is likely to form glass. Most of the metal oxide generally doesn't follow Zachariasen rule and are called network modifier. $\mathrm{Li}_{2} \mathrm{O}$ belongs to the latter group because of its highly charged cations 
oxide, small ionic radii and cations are tetrahedral coordinates to oxygen. Phosphorus pentoxide is, however, a network former. The phosphate glass structure is formed by $\mathrm{PO}_{4}$ tetrahedra connected to each other by corners. Glass $\mathrm{P}_{2} \mathrm{O}_{5}$ creates a 3-D network of $\mathrm{PO}_{4}$ tetrahedra connected by three of the four corner oxygen while the other oxygen shares double-bonds with another $\mathrm{P}$. When $\mathrm{Li} 2 \mathrm{O}$ is mixed with $\mathrm{P}_{2} \mathrm{O}_{5}$, it depolymerizes the phosphate chain by breaking some of the P-O-P corner bonds to capture the $\mathrm{Li}$ and shorten the chains.

In Fig 27 such a glass structure with 200 atoms is shown forming a small chain of $\mathrm{PO}_{4}$ tetrahedral where two of most of the phosphate's corner oxygen is shared with another tetrahedral oxygen while Li ions are floating randomly in the space surrounded with oxygen by electrostatic force. We will see that these single bonded oxygens create a tunnel for $\mathrm{Li}$ to diffuse. In this study, we have considered a molecule of $0.6 \mathrm{Li}_{2} \mathrm{O}_{5}-0.4$ $\mathrm{P}_{2} \mathrm{O}_{5}$ glass. The lack of a long-range order makes the Li diffusion path very difficult to predict in the glassy material. Several theoretical studies have been done in this field to understand the total interaction processes. B.K. Money [38] used a reverse[104] Monte Carlo modeling to show the most probable path of Li trajectory and reported a fast ion transport in the glass which could have resulted from the hopping of mobile ions between various vacant site through these pathways [38]. Other modeling approaches [105] reported a similar ion migration to occur through the "interstitial pair formation" mechanism. In a couple of recent studies [51], [52], AIMD simulations were employed to investigate ion movement in $\mathrm{Si}_{2} \mathrm{O}_{5}$ and showed ion trajectories to be divided into several modes associated with vibrations at a fixed site and hopping between sites. 

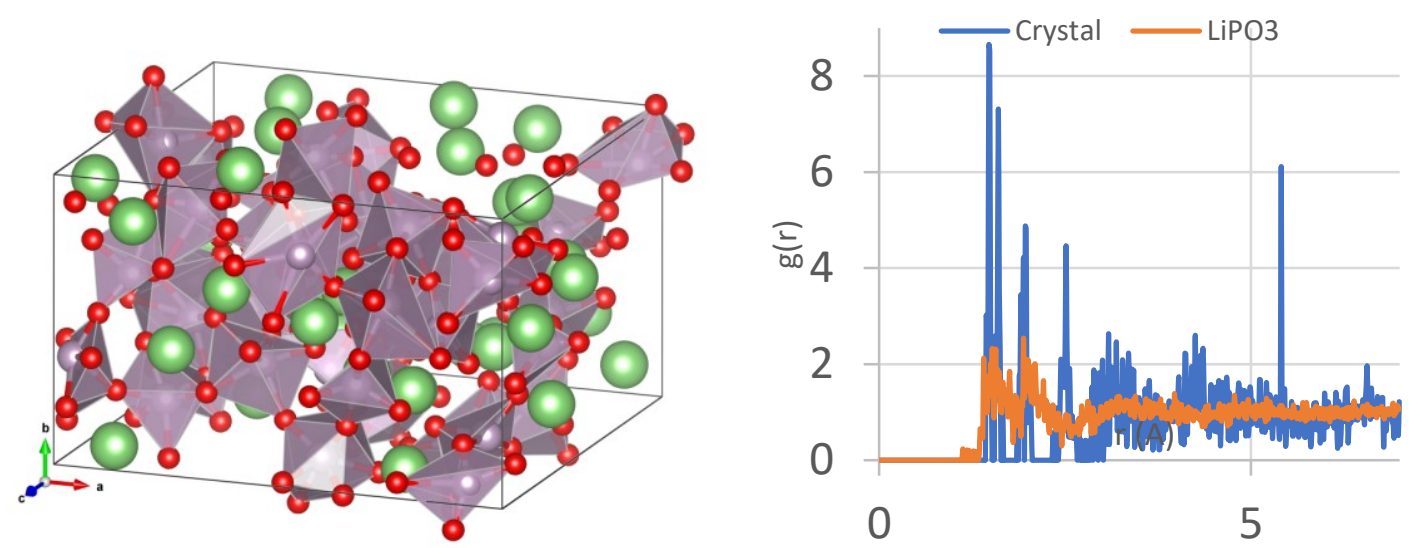

Fig 27. a) $50 \% \mathrm{Li}_{2} \mathrm{O}-50 \% \mathrm{P}_{2} \mathrm{O}_{5}$ supercell containing interconnecting $\mathrm{P}-\mathrm{O}$ polyhedral cluster containing $24.59 \%$ volume of the total space. b) RDF analysis of crystal and amorphous structure showing the disappearance of long-range order.

\section{AIMD Simulations}

For glass structure analysis, we have used a plane wave cut-off energy of $275 \mathrm{eV}$ and the maximum ionic optimization force is set to $\leq 0: 02 \mathrm{eV}^{\circ} \mathrm{A}-1$. A Verlet algorithm is used to integrate Newton's equations of motion with a $1 \mathrm{fs}$ time step. For MD calculation, $\Gamma$-point sampling of Brillouin zone is enough. To create amorphous structure, we increased the $1 \times 2 \times 1$ relaxed super-cell temperature to $3000 \mathrm{~K}$ with the canonical ensemble (NVT) in 0.8 ps to melt the structure, allowing volume change due to phase transformation. To minimize the transition state raddling and stabilize the structure, we run it for 1ps. Fig 27.b shows a comparison of the radial distribution function (RDF) analysis how the ordering disappears in the amorphous structure. The initial velocity is set randomly according to a Maxwell-Boltzmann distribution. The structure was then cooled down with a cooling rate of $2: 5 \times 1015 \mathrm{~K} / \mathrm{s}$ and the volume is again optimized to prepare a sample for next simulation run. 


\section{Method Procedure}

For a consistent AIMD simulation under a constant volume condition, the phase transition such as melting process needs to be evaluated first so that the characteristic change in the simulation structure as depicted by the range of pressure and energy to mark the amorphous/molten state can be identified. These two parameters will obviously change during melting. The axial pressure indicator is also a good way to detect the volumetric change in particular direction for the simulation purpose. An upper bound of $10 \mathrm{GPa}$ pressure is maintained in all direction and a 5\% reduction in volume is required to optimize the structure. This same process is used to optimize the structure after any temperature change of the structure for calculating the potential energy and free energy. Considering the time constraint in AIMD process and to better understand the $\mathrm{Li}$ diffusion process, we run a number of isothermal processes at higher temperatures to assess the diffusion mechanism. The change in the diffusivity with time can provide a hint of the inherent mechanisms as associated with the temperature effect. We would reasonably expect a linear change of a log of diffusivity with temperature indicative of Arrhenius relationship. To obtain diffusivity in a number of targeted temperatures; 3000 , 2500, 2000, $1500 \mathrm{~K}$, AIMD simulation is performed with an NVT ensemble. As indicated by the Einstein relation, MSD can be related to the diffusivity D. The MSD of particular ion like $\mathrm{Li}^{+}, \mathrm{P}^{5+}$, and $\mathrm{O}^{2-}$ can be calculated using the relation. For each of the isothermal calculation, the diffusion coefficient of Lithium is measured to check the variation of diffusivity with temperature. From this information, the Arrhenius plot is drawn to find the activation energy of Li-ion diffusion. At the same time, the direct current conductivity is also calculated from the random diffusion coefficient (D) using 
the Nernst-Einstein relation which shows the $\mathrm{Li}+$ conductivity can be vastly different for $\mathrm{Li}$ compositions e.g in $\mathrm{Li}_{2} \mathrm{O}, \mathrm{P}_{2} \mathrm{O}_{5}$. It also showed the $\mathrm{Li}$ conductivity increases along with the increasing fraction of $\mathrm{Li}_{2} \mathrm{SO}_{4}$ and decreases after a certain limit. Ref [95] predicts the increase in the $\mathrm{Li}$ content ratio (1:4 to 1:3.25). It was argued that presence of ultramicroscopic cluster-tissue texture is the sole reason for this phenomenon. To test this idea, a model of supercell of $60 \% \mathrm{Li}_{2} \mathrm{O}-\mathrm{P}_{2} \mathrm{O}_{5} 40 \% \mathrm{Li}_{2} \mathrm{SO}_{4}$ is created. This is achieved by substituting $40 \%$ of $\mathrm{P}$ with $\mathrm{S}$ atom in the $\mathrm{Li}_{2} \mathrm{O}-\mathrm{P}_{2} \mathrm{O}_{5}$ model amorphous structure. The addition of $\mathrm{Li}_{2} \mathrm{SO}_{4}$ leads to an increase in the $\mathrm{Li}$ ionic concentration. At the same time, because $\mathrm{S}$ atoms show an increase of the valence state from $\mathrm{P}^{5+}$ to $\mathrm{S}^{+6}, \mathrm{O}$ and $\mathrm{Li}$ are also carefully added into the simulation box to maintain the charge balance inside the total cell. $\mathrm{O}$ atoms are placed in a close proximity to $\mathrm{S}$ atoms whereas the $\mathrm{Li}$ atoms are placed adjacent to the $\mathrm{O}$ atoms, maintaining the normal first-order $\mathrm{RDF}$ distance (1.45 $\mathrm{A}$ and 1.65 A, respectively). AIMD isothermal approach is applied on this structure for $1 \mathrm{ps}$ to let the newly added $\mathrm{S}, \mathrm{O}$ and $\mathrm{Li}$ atoms freely roam and to remove an initial bias caused by the initial placement of these atoms within the structure. The inclusion of new atoms increases the volume and the breakage of the polyhedral chain, which is quite noticeable. These characteristic changes can be explained by the valance difference of $\mathrm{S}^{6+}$ and $\mathrm{P}^{5+}$ and a higher electron affinity of S relative to that of P. Fig 28. a show the electron density of $\mathrm{SO}_{4}$ and $\mathrm{PO}_{4}$ bonds where strong electron correlation of $\mathrm{S}$ makes double bond with 2 $\mathrm{O}$ which make these $\mathrm{O}$ inert to make another bond with surrounding cations. Letting only 2 corner oxygen to bond with ether $\mathrm{Li}$ or corner $\mathrm{O}$ of $\mathrm{PO}_{3}$ tetrahedral and hence creating isolated self-centered tetrahedral where 3 of 4 corners of $\mathrm{PO}_{3}$ tetrahedral tends to share $\mathrm{O}$ with other entity. Fig 288 a \& b show an average coordination number $(\mathrm{CN})$ analysis 
over the dynamic trajectory steps. This shows for a particular type material; the $\mathrm{CN}$ maintains an average value of the SD to be 0.02 for $\mathrm{P}$ in pure and 0.367 for $\mathrm{P}$ in the mix. A reduction of $\mathrm{CN}$ from 2.79 to 1.267 of $\mathrm{P}$ is also noticeable from pure $\mathrm{LiPO}_{3}$ to a mixed composition. This reduction can be explained by the fact that the substituting $\mathrm{S}$ in the initial stage would seek the shared $\mathrm{O}$, initially connected to $\mathrm{P}$ and break out from the long chain of $\mathrm{P}$ polyhedral cluster, making it's own polyhedral leading to the overall breakage of the long-chain $\mathrm{P}$-dominated cluster.
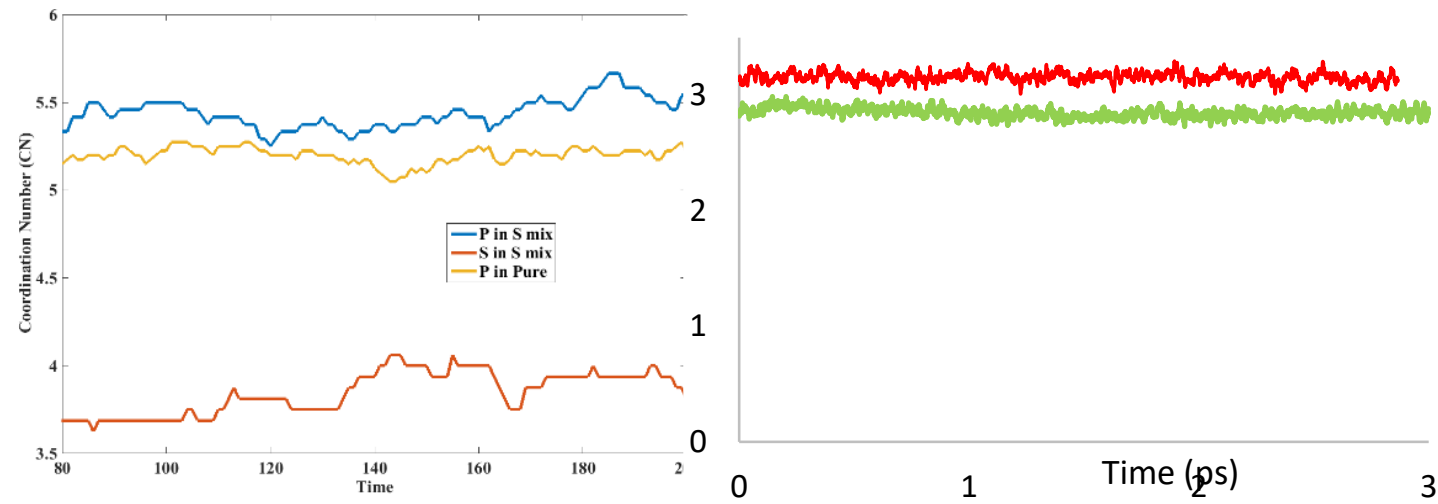

Fig 28. a) Electron density map around $\mathrm{S}$ and $\mathrm{P}$ in $60 \%\left(\mathrm{Li}_{2} \mathrm{O}-\mathrm{P}_{2} \mathrm{O}_{5}\right) 40 \% \mathrm{Li}_{2} \mathrm{SO}_{4}$. b) Coordination number analysis of $\mathrm{P} \& \mathrm{~S}$ c) $\mathrm{CN}$ analysis of $\mathrm{Li}$ in both structures. 


\begin{abstract}
ANALYSIS
An equilibrated amorphous $\mathrm{LiPO}_{3}$ model is shown in Fig 27 in comparison with the crystalline super-cell. The radial distribution (RDF) of crystalline structure at ground state and $\mathrm{LiPO}_{3}$ at $3000 \mathrm{~K}$ indicates the loss of the crystalline state at $3000 \mathrm{~K}$. The smoothing of total $\mathrm{g}(\mathrm{r})$ after $2 \mathrm{~A}$ in amorphous medium suggests the disappearance of a long-range order where the first two peaks if compared to the individual bond RDFs, would correspond to the first order peak of $\mathrm{P}-\mathrm{O} \& \mathrm{Li}-\mathrm{O}$ and $\mathrm{O}-\mathrm{O}$ first nearest neighbor on each case. It is noticeable that even after shrinking of crystal structure in the glassy state, the O-O and Li-O distance would shorten considerably while the P-O tetrahedral formation remains relatively unchanged. The chain of tetrahedral clusters breaks into smaller clusters of polyhedral connected to each other. The relative space occupation of polyhedral calculation shows an increase from $3.09 \%$ to $24.59 \%$ volume occupation by the $\mathrm{P}-\mathrm{O}$ polyhedron whenever $\mathrm{Li}-\mathrm{O}$ and $\mathrm{O}-\mathrm{O}$ bond spacings are reduced. Considering the analogous to the Li migration in crystal $\mathrm{LiPO}_{3}$, the surrounding oxygen in tetrahedral is the key to achieve a low activation energy of the Li migration. Moreover, from the isothermal AIMD simulations, a close observation on the movement of the Li migration suggests that the same mechanism can be detected with the exception that the higher temperature conditions create a localized vibration and the migration also involves a relatively lower frequency vibration. This process is illustrated by the aggregated trajectory image in Fig 29 for 2 random diffusive Li-ions. The picture clearly shows the Li-O bonding tracks and how the intermediate Li-O bond exchange takes place between two site migrations. A successful migration occurs when the Li-O distance from one site
\end{abstract}


reaches a critical distance and another $\mathrm{Li}-\mathrm{O}$ distance from the opposite site shortens to generate a new Li-O bond in the interim process. For this specific case, the thermal vibration may provide the necessary energy or force to the Li atom to raddle around the localized position and the migration process occurs when its trajectory is surrounded by oxygen atoms with a sufficient distance so that it can create a new bond with the migrating Li-ion. This appears to reduce the migration barrier and helps Li to overcome the ionic force and brake the existing Li-O bond. In the AIMD processes that we generated, several Li-ions do remain localized in a relatively short 3 ps timeline. This doesn't necessarily prove that those will remain localized for the extended timeframe.
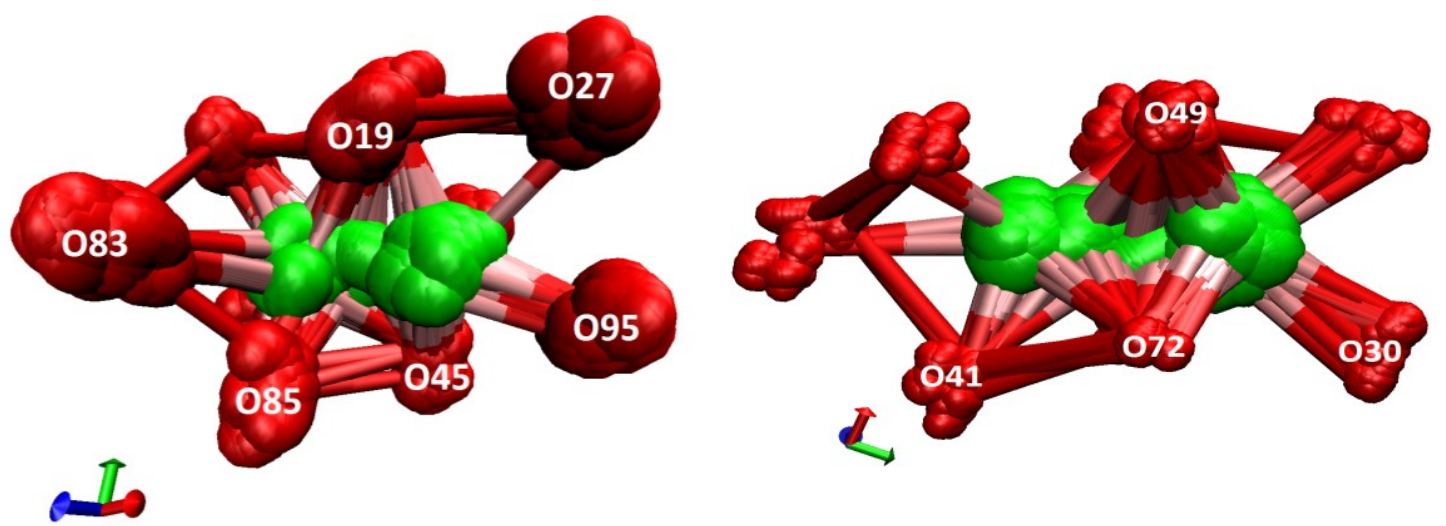

Fig 29. Li migration for 2 random Li, with Li-O bond handover process in $\mathrm{LiPO}_{3}$ amorphous structure. Green indicates $\mathrm{Li}$ and red $\mathrm{O}$ atom.

In case of $\mathrm{Li}_{2} \mathrm{O}-\mathrm{P}_{2} \mathrm{O}_{5}-\mathrm{Li}_{2} \mathrm{SO}_{4}, \mathrm{P}_{2} \mathrm{O}_{5}$ chain breaks down and form several monopolyhedral clusters as shown in Fig 30. This leads to an increase in empty space within the structure, but calculation shows the fixed tetrahedral occupies only $15.344 \%$ of the total space. This suggests the creation of enough empty space for Li to diffuse. Moreover, S mix sample has Li's CN 2.87 whereas the Li in pure sample shows a 3.17 coordination number. Under Pauling's rule, the effective radius of the cation is larger when the $\mathrm{CN}$ is 
higher. This suggests that the Li-ion is less ionic (with a larger radius) in $\mathrm{LiPO}_{3}$ making $\mathrm{Li}$ harder to diffuse. However, we will see having only an empty space is not a sufficient condition for a higher diffusion. The surrounding condition is also a major factor and can play a major role. The RDF analysis on the mix structure shows a right shift (i.e. toward a larger distance) of $\mathrm{Li}-\mathrm{O}$ and $\mathrm{O}-\mathrm{O}$ bond distances compared to that obtained from the $\mathrm{Li}_{2} \mathrm{O}-\mathrm{P}_{2} \mathrm{O}_{5}$ with a magnitude of 0.1 and 0.2 A respectively.

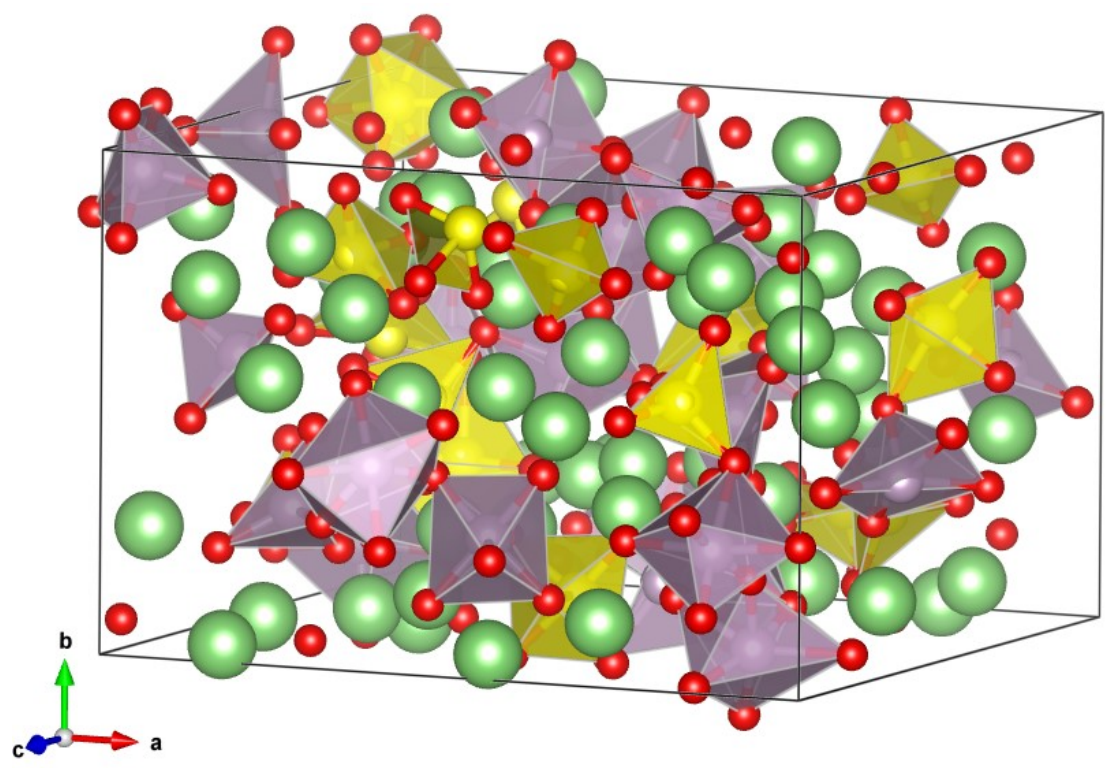

Fig 30. LiPO3SO4 supercell showing a cluster of P-O polyhedral (violet) and isolated SO tetrahedral (yellow). Theses structure consumes $15.344 \%$ of total supercell space.

To understand the effect of this structure on Li diffusion perspective, MSD of an individual element is calculated. As the initial random distribution of velocity might cause non-physical velocity distributions, the MSD is calculated after 0.5 ps stabilization period. Fig 31 shows an average MSD data for $\mathrm{Li}^{+}, \mathrm{P}^{5+}, \mathrm{S}^{6+}, \mathrm{O}^{2-}$ over an $11.8 \mathrm{ps}$ timeline at $3000 \mathrm{~K}$. The MSD graph with a steeper slope (blue) suggests rapid movement where a horizontal slope suggests no movement at all. From this graph, we see that $\mathrm{Li}$ is more 
diffusive than the surrounding $\mathrm{P}, \mathrm{S}$ or $\mathrm{O}$. The same approach is applied for $\mathrm{Li}_{2} \mathrm{O}-\mathrm{P}_{2} \mathrm{O}_{5}$ and the result is illustrated in Fig 32 showing an increase of Li diffusion over time even though the total diffusivity is less than the 60-40 mix. In addition, the diffusivity in different temperatures are also measured. From the Arrhenius equation, we know there is a direct relationship between an energy barrier and the diffusivity as a function of temperate and if we plot $\operatorname{Ln}(\mathrm{D})$ vs $1 / \mathrm{T}$ graph, the steepness of slope represents the magnitude of the energy barrier of the ion transport. One particular analysis had been done on $\mathrm{LiPO}_{3} \& \mathrm{~S}_{\text {mix }} \mathrm{LiPO}_{3}$ glass material[2]. They showed the energy barrier increases with an increase in temperature (Table 9). The study was performed in $250 \&$ $500 \mathrm{~K}$ which shows a significant increase in energy barrier. However, this trend is also true for the S mix sample. Due to the time limitation in the DFT analysis, I have calculated the energy barrier in higher temperatures. As shown in Fig 33, calculation shows a $0.62 \mathrm{eV}$ energy barrier which is $0.09 \mathrm{eV}$ more than that obtained from the experimental result[2] but considering the trend of increasing energy barrier, the pattern seems the same.

Table 9: Energy barrier comparison with experimental work for pure Li2O-S2O5 and 60$40 \mathrm{Li} 2 \mathrm{O}-\mathrm{P} 2 \mathrm{O} 5-\mathrm{Li} 2 \mathrm{SO} 4$ amorphous structure

\begin{tabular}{|l|l|l|}
\hline Temperature(K) & $\begin{array}{l}\text { Energy barrier of Li2O- } \\
\text { P2O5(eV) }\end{array}$ & $\begin{array}{l}\text { Energy barrier of S mix } \\
\text { sample(eV) }\end{array}$ \\
\hline 250 & $0.06[2]$ & $0.02[2]$ \\
\hline 500 & $0.53[2]$ & $0.39[2]$ \\
\hline 3000 & 0.62 (this study) & 0.41 (this study) \\
\hline
\end{tabular}




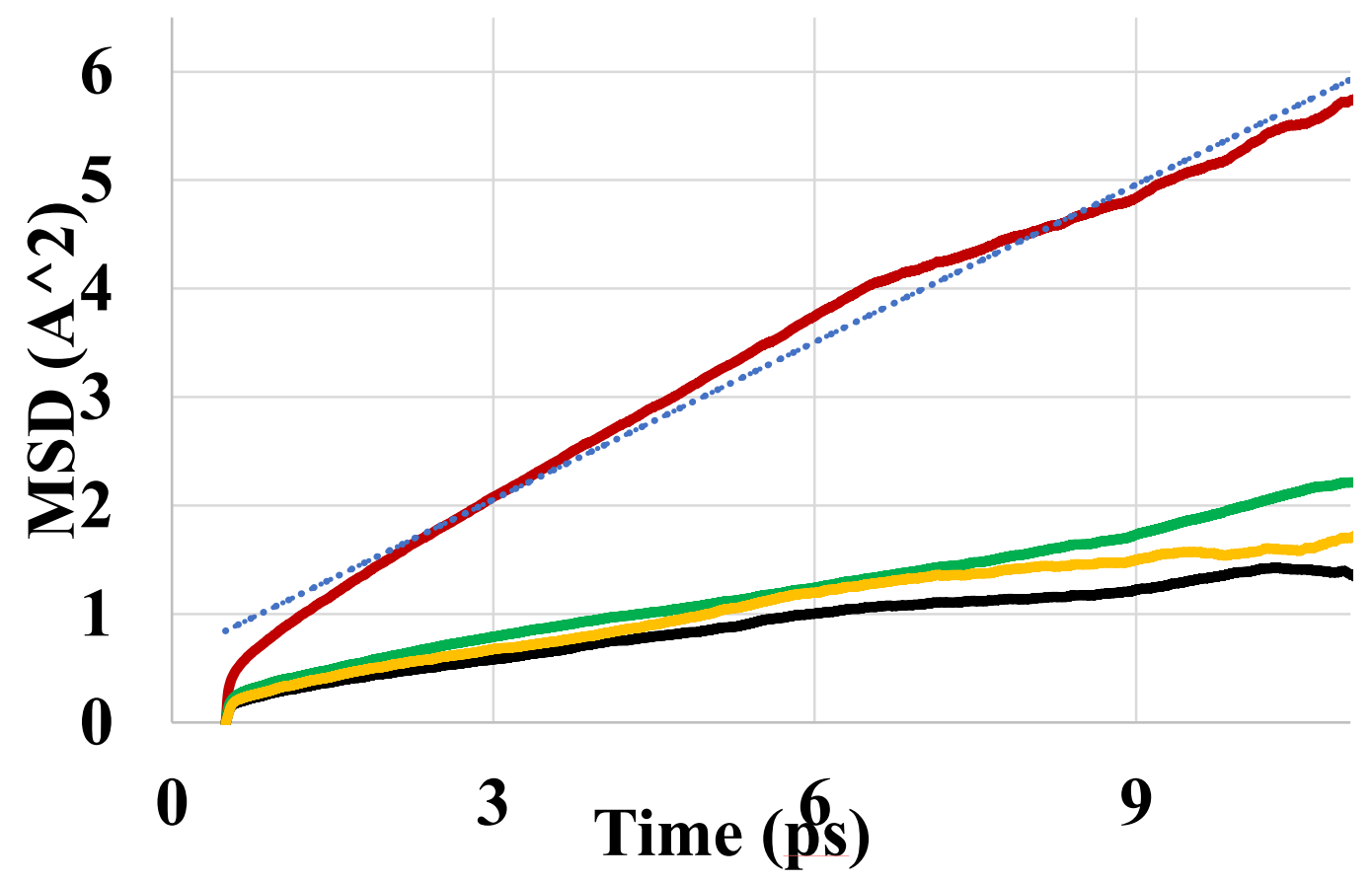

Fig 31. Average MSD of a different element in Li2P205SO4 at 3000K for $12 \mathrm{ps.}$

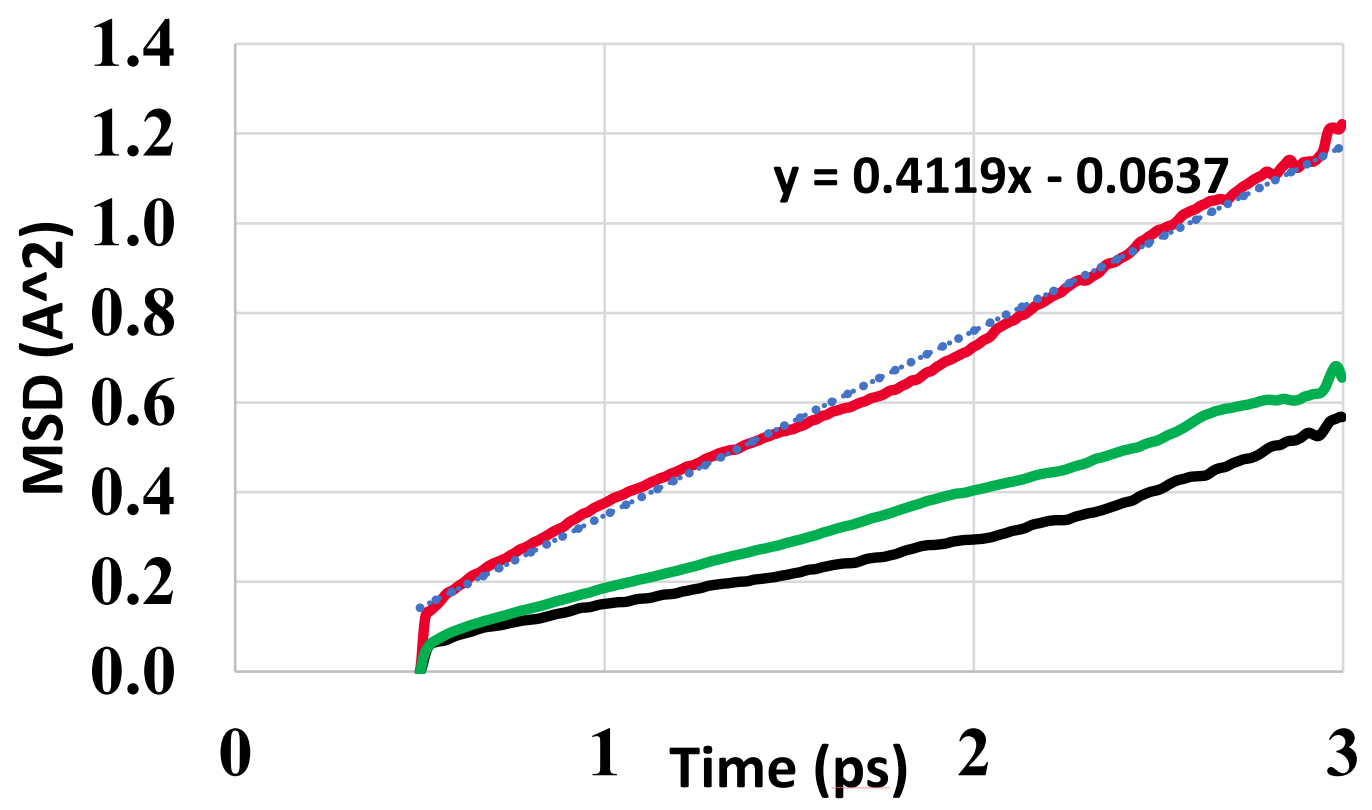

Fig 32. Average MSD of different element in Li2P2O5 at 3000K for 3 ps. 


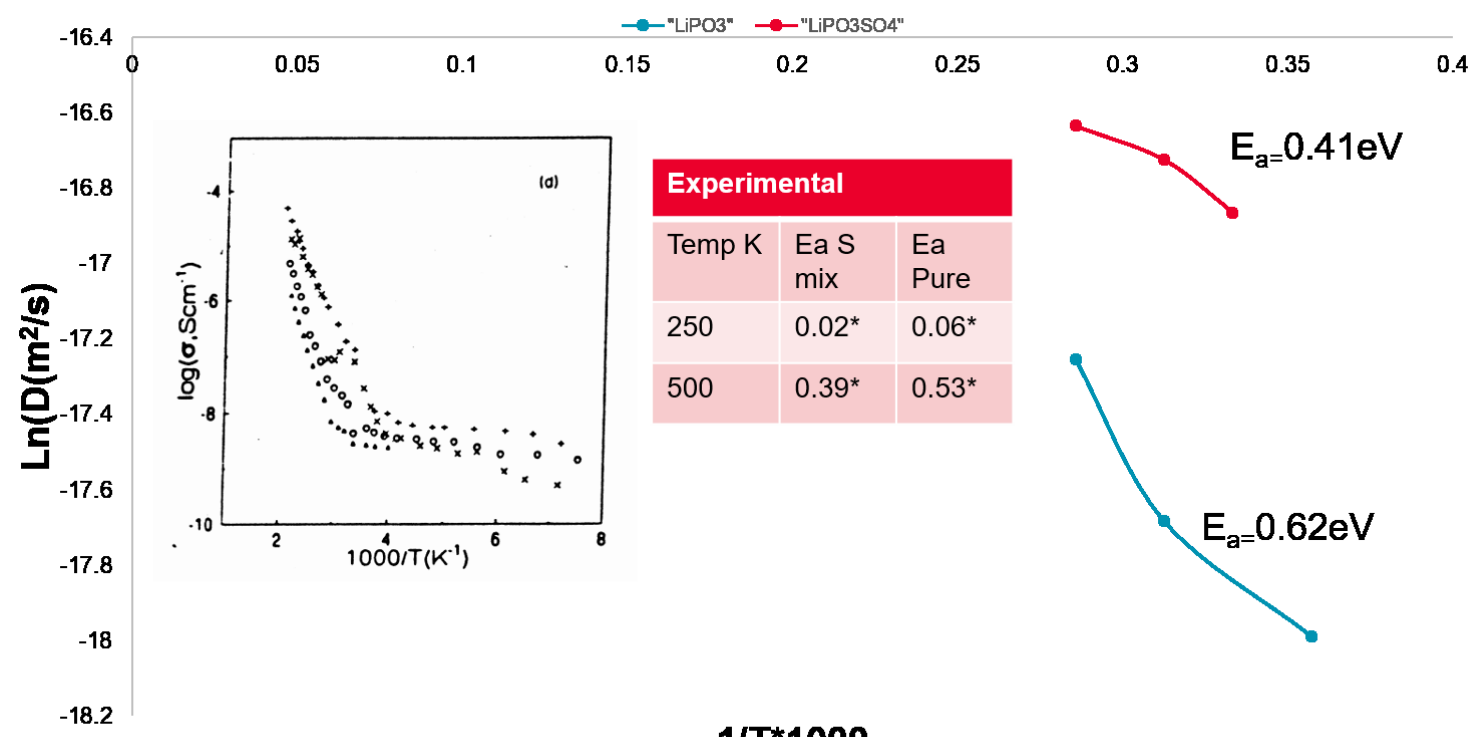

Fig 33. Diffusivity vs $1 /$ T graph. Showing comparison of energy barrier with experimental result

Movements of the Li ions are closely analyzed to check if the Li-O bond exchange mechanism is applicable for the mix structure in migration time. Fig $34 \mathrm{~s}[\mathrm{a}, \mathrm{b}]$ shows the dynamic Li-O bond handover process in the migration time considering 2 separate conditions: 1) migration aided by multiple oxygens as Li moves through the path \& 2) migration controlled by a set of the oxygen atom. Fig 34.b shows how 2 oxygen atoms guiding the $\mathrm{Li}$ atom to migrate from one site to another site where the other bond was broken and re-created after the migration process. The thick two corners show the localized vibration of Li atom before the migration and the path become narrow at the migration time. This migration however was made easier with the presence of $\mathrm{O}$ atoms in the intermediate space particularly O128 and O46. Without the presence of these Os, the saddle point would show a much higher activation energy as all the bonds between Li-O had to completely break before the diffusion process to proceed. In this particular 
structure, the intermediate $\mathrm{O}$ creates a bond with the Li releasing the energy as a consequence which helps to keep the activation energy much lower and these intermediate O's keep the bond until the Li gets migrated to the new site. However, the presence of only $2 \mathrm{O}$ atoms makes this site a transition site and Li need to diffuse further to find a favorable site.

The vibrational analysis is also a good way to understand the structure and migration process. The vibrations in a dense structure supposedly will show a higher frequency than its low-density material and when an element gets a favorable condition to move from one region to another, the intermediate opening of low potential space reduces the obstacle for movement results in a lower down its frequency. Fig 35 shows the vibrational frequency of all the elements $(\mathrm{Li}, \mathrm{O}, \mathrm{P}, \mathrm{S})$ in $\mathrm{Li}_{2} \mathrm{O}-\mathrm{P}_{2} \mathrm{O}_{5}-\mathrm{Li}_{2} \mathrm{SO}_{4}$ and in $\mathrm{Li}_{2} \mathrm{O}-\mathrm{P}_{2} \mathrm{O}_{5}$ composition.
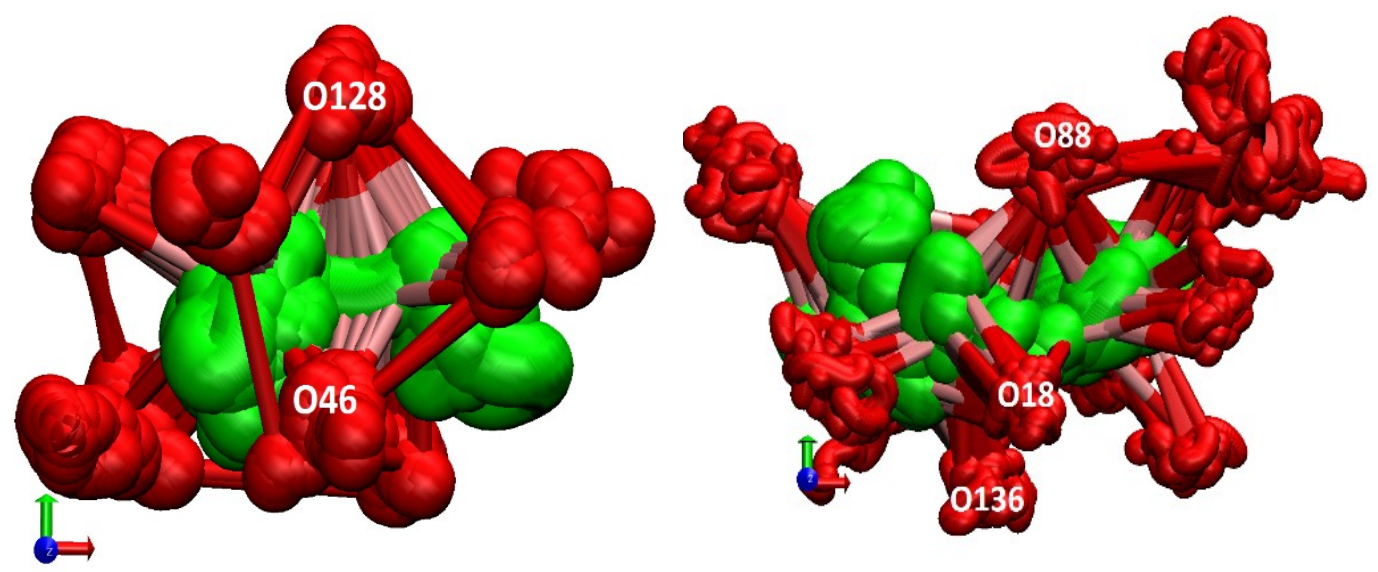

Fig 34. Li migration with $\mathrm{Li}-\mathrm{O}$ bond handover process in $\mathrm{LiPO}_{3} \mathrm{SO}_{4}$. The green mesh shows the movement of $\mathrm{Li}$ and the red-pink bar showing the dynamic $\mathrm{Li}-\mathrm{O}$ bond created in the intermediate stages. It also shows the $\mathrm{O}$ atoms (red mesh) are localized during the migration a) a long migration where several $\mathrm{O}$ contribute to the migration b) site to site migration contributed by only 2 oxygen atom. 
We can observe, except Li, the vibrational frequency of the other elements does not have any significant change of DOS occupancy, whereas, for Li, the peak frequency does have a left shift toward a lower frequency. The graphs show a band of the vibration frequency of Li for both of the structure with different range and a left shift of the band for S mix structure is consistent with the low-frequency prediction. The change in low frequency also suggest an increased bond length and lower dense structure[106]. In [106], Kitagawa et.al. showed that how the stress shifts the raman band and found out the reason is interatomic change of bond. However, a wide peak in my data suggests the amorphous of the structure. In [107], David Tuschel has discussed the effect of periodicity over the vibrational characteristics of the material. Also, he suggested the lacking of periodicity is causing the long-range smear of the vibrational peak. For $\mathrm{LiPO}_{3}$, after addition of sulfur content, Oxygen, Phosphorus, shows some broad peak, this may lead to the trend of periodicity due to the addition of sulfur.
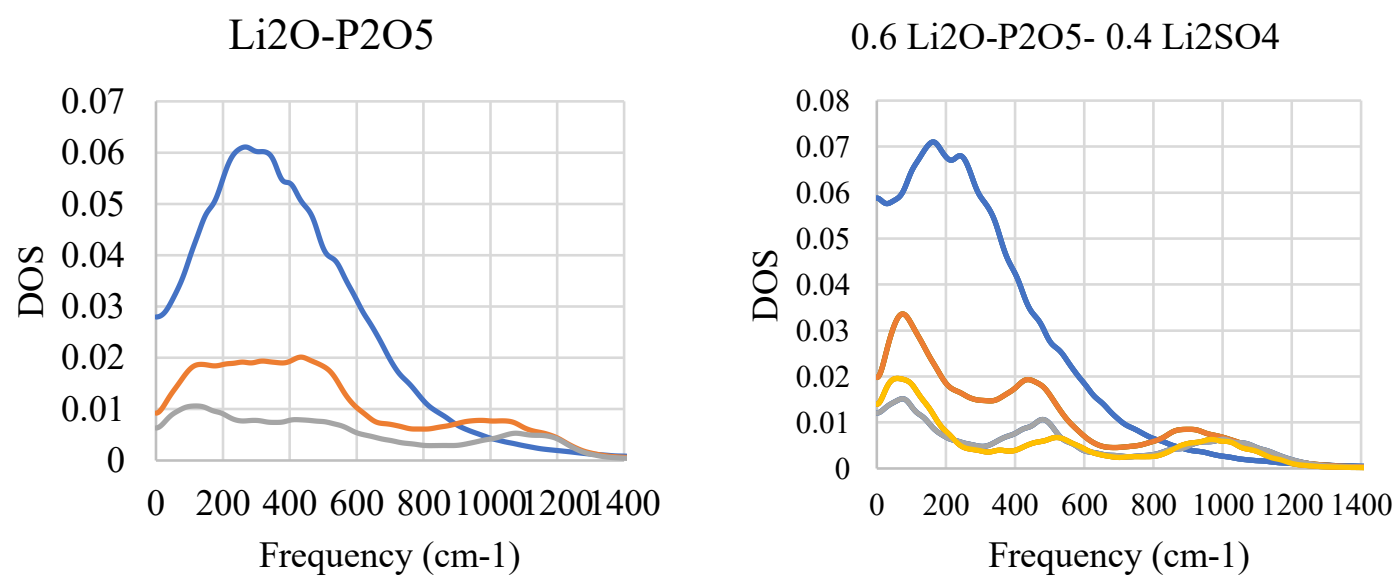

Fig 35. a) Vibration frequency of $\mathrm{Li}$ (green), $\mathrm{O}$ (orange), $\mathrm{P}$ (gray), $\mathrm{S}$ (yellow) in $\mathrm{LiPO}_{3}$ amorphous medium and b) in $\mathrm{Li} 2 \mathrm{P} 2 \mathrm{O} 5 \mathrm{Li}_{2} \mathrm{SO}_{4}$. Total $\mathrm{Li}$ frequency band shifted to the left in S mix one. 
Table 10. Experimental result of conductivity and energy barrier for different composition [2]

\begin{tabular}{|c|c|c|c|}
\hline \multirow[t]{2}{*}{ Sample } & \multirow{2}{*}{$\begin{array}{l}\sigma(298 \mathrm{~K}) \\
(\mathrm{S} / \mathrm{cm})\end{array}$} & \multicolumn{2}{|l|}{$E_{\mathrm{a}}(\mathrm{dc})$} \\
\hline & & $\begin{array}{l}\text { High temp. } \\
(\mathrm{eV})\end{array}$ & $\begin{array}{l}\text { Low temp. } \\
(\mathrm{eV})\end{array}$ \\
\hline CLSP1 & $1.3 \times 10^{-7}$ & 0.39 & 0.02 \\
\hline CLSP2 & $1.4 \times 10^{-7}$ & 0.37 & 0.03 \\
\hline CLSP3 & $2.0 \times 10^{-7}$ & 0.39 & 0.02 \\
\hline CLSP4 & $4.2 \times 10^{-7}$ & 0.39 & 0.06 \\
\hline CLOPl & $4.3 \times 10^{-9}$ & 0.40 & 0.02 \\
\hline CLOP2 & $1.9 \times 10^{-8}$ & 0.31 & 0.02 \\
\hline CLOP3 & $2.8 \times 10^{-7}$ & 0.39 & 0.07 \\
\hline CLOP4 & $4.2 \times 10^{-7}$ & 0.39 & 0.06 \\
\hline $\mathrm{CPl}$ & $6.4 \times 10^{-8}$ & 0.40 & 0.10 \\
\hline $\mathrm{CP} 2$ & $1.1 \times 10^{-7}$ & 0.39 & 0.05 \\
\hline $\mathrm{CP} 3$ & $3.8 \times 10^{-7}$ & 0.35 & 0.02 \\
\hline $\mathrm{CP} 4$ & $1.3 \times 10^{-7}$ & 0.39 & 0.02 \\
\hline CLP1 & $2.8 \times 10^{-9}$ & 0.53 & 0.06 \\
\hline CLP2 & $4.3 \times 10^{-9}$ & 0.40 & 0.02 \\
\hline CLP3 & $8.0 \times 10^{-8}$ & 0.39 & 0.06 \\
\hline CLP4 & $1.3 \times 10^{-7}$ & 0.39 & 0.02 \\
\hline
\end{tabular}




\section{CONCLUSION}

In my study, I have reproduced previously reported energy barriers in different paths for $\mathrm{Li}_{3} \mathrm{PO}_{4}$ with a significant accuracy using the Nudge elastic band method. I have also analyzed the energy barrier of different paths in $\mathrm{LiPO}_{3}$ and found that $\mathrm{LiPO}_{3}$ has a suitable environment for Li to diffuse in a much lower barrier $(0.36 \mathrm{eV}$ vs $0.294 \mathrm{eV}$ in $\left.\mathrm{LiPO}_{3}\right)$ in the crystalline material. With these crystalline analyses, I have performed abinitio molecular dynamics (AIMD) analysis to calculate the Li diffusivity in amorphous $\mathrm{LiPO}_{3}$ structure. The result shows that $\mathrm{Li}$ is much mobile than other elements in the structure. I have also shown that the addition of $\mathrm{Li}_{2} \mathrm{SO}_{4}$ in the $\mathrm{LiPO}_{3}$ amorphous structure increases the Li diffusivity in the structure as demonstrated by the increase in MSD value which is consistent with the reported experimental results. The AIMD simulation reveals the partial breakup of long phosphate polyhedral clusters making more space for $\mathrm{Li}+$ ion to diffuse much easier. Along with this analysis, the vibrational analysis on the trajectory of $\mathrm{Li}+$ ion shows a lowering of vibrational frequency of $\mathrm{Li}$ ion in $\mathrm{S}$ mix sample. I have also observed the possible role of the nearest neighbor oxygens in assisting the $\mathrm{Li}+$ ion hopping from site to site and how it is important to have a higher $\mathrm{CN}$ for the lower barrier. At last, I analyzed the role of Li-O bond with the energy of the system and observed how the Li-O bond length in the TS and IS plays role in the barrier of the Li migration. I also have observed that there is no particular pattern of EB with the average Li-O bond of the TS migrating atom in $\mathrm{Li}_{3} \mathrm{PO}_{4}$ structure and the evaluation of IS and TS need to be considered to predict the EB. 


\section{REFERENCES}

[1] Y. a. Du and N. a. W. Holzwarth, "Li Ion diffusion mechanisms in the crystalline electrolyte $\gamma$-Li[sub 3]PO[sub 4]," J. Electrochem. Soc., vol. 154, no. 11, p. A999, 2007.

[2] M. Ganguli, M. H. Bhat, and K. . Rao, "Lithium ion transport in Li2SO4-Li2OP2O5 glasses," Solid State Ionics, vol. 122, no. 1, pp. 23-33, 1999.

[3] B. Dunn, H. Kamath, and J.-M. Tarascon, "Electrical energy storage for the grid: a battery of choices," Science (80-. )., vol. 334, no. 6058, pp. 928-935, Nov. 2011.

[4] Z. Yang et al., "Electrochemical energy storage for green grid," Chemical Reviews, vol. 111, no. 5. pp. 3577-3613, 2011.

[5] A. Manthiram, Y. Fu, and Y. S. Su, "In charge of the world: Electrochemical energy storage," J. Phys. Chem. Lett., vol. 4, no. 8, pp. 1295-1297, 2013.

[6] M. Pasta, C. D. Wessells, R. A. Huggins, and Y. Cui, "A high-rate and long cycle life aqueous electrolyte battery for grid-scale energy storage," Nat. Commun., vol. 3, no. May, p. 1149, 2012.

[7] V. Thangadurai, S. Narayanan, and D. Pinzaru, "Garnet-type solid-state fast Li ion conductors for Li batteries: critical review.," Chem. Soc. Rev., vol. 43, no. 13, pp. 4714-27, 2014.

[8] J. Liu et al., "Materials science and materials chemistry for large scale electrochemical energy storage: From transportation to electrical grid," Adv. Funct. Mater., vol. 23, no. 8, pp. 929-946, 2013.

[9] X. Ning, S. Phadke, B. Chung, H. Yin, P. Burke, and D. R. Sadoway, "Selfhealing Li-Bi liquid metal battery for grid-scale energy storage," J. Power Sources, vol. 275, pp. 370-376, 2015.

[10] M. D. Bhatt and C. O'Dwyer, "Recent progress in theoretical and computational investigations of Li-ion battery materials and electrolytes," Phys. Chem. Chem. Phys., vol. 17, no. 7, pp. 4799-4844, 2015.

[11] F. Zhou, M. Cococcioni, K. Kang, and G. Ceder, "The Li intercalation potential of LiMPO4 and LiMSiO4 olivines with $\mathrm{M}=\mathrm{Fe}, \mathrm{Mn}, \mathrm{Co}, \mathrm{Ni}$," Electrochem. commun., vol. 6, no. 11, pp. 1144-1148, 2004. 
[12] G. Li, H. Azuma, and M. Tohda, "LiMnPO4 as the cathode for lithium batteries," Electrochem. Solid-State Lett., vol. 5, no. 6, pp. A135--A137, 2002.

[13] K. Amine, H. Yasuda, and M. Yamachi, "Olivine LiCoPO4 as 4.8 V Electrode material for lithium batteries," Electrochem. Solid-State Lett., vol. 3, no. 4, pp. $178-179,2000$.

[14] A. Manthiram, "Materials challenges and opportunities of lithium ion batteries," pp. 176-184, 2011.

[15] M. S. Islam and C. A. J. Fisher, "Lithium and sodium battery cathode materials. Computational insights into voltage, diffusion and nanostructural properties.," Chem. Soc. Rev., vol. 43, no. 1, pp. 185-204, 2014.

[16] F. L. and H.-M. C. Guangmin Zhou, "Progress in flexible lithium batteries and future prospects," pp. 1307-1338, 2014.

[17] K. Takahashi et al., "All-solid-state lithium battery with LiBH4 solid electrolyte," J. Power Sources, vol. 226, pp. 61-64, 2013.

[18] A. . Robertson, A. . West, and A. . Ritchie, "Review of crystalline lithium-ion conductors suitable for high temperature battery applications," Solid State Ionics, vol. 104, no. 1, pp. 1-11, 1997.

[19] G. Adachi, N. Imanaka, and H. Aono, "Fast Li $\oplus$ conducting ceramic electrolytes," Adv. Mater., vol. 8, no. 2, pp. 127-135, Feb. 1996.

[20] S. Stramare, V. Thangadurai, and W. Weppner, "Lithium lanthanum titanates: A Review," Chem. Mater., vol. 15, no. 21, pp. 3974-3990, 2003.

[21] J. F. Ihlefeld, P. G. Clem, B. L. Doyle, P. G. Kotula, K. R. Fenton, and C. A. Apblett, "Fast lithium-ion conducting thin-film electrolytes integrated directly on flexible substrates for high-power solid-state batteries," Adv. Mater., vol. 23, no. 47, pp. 5663-5667, 2011.

[22] H. Buschmann et al., "Structure and dynamics of the fast lithium ion conductor 'Li7La3Zr2O12,'” Phys. Chem. Chem. Phys., vol. 13, no. 43, p. 19378, 2011.

[23] R. Djenadic et al., "Nebulized spray pyrolysis of Al-doped Li7La3Zr2O12 solid electrolyte for battery applications," Solid State Ionics, vol. 263, pp. 49-56, 2014.

[24] R. Murugan, V. Thangadurai, and W. Weppner, "Fast lithium ion conduction in garnet-type Li(7)La(3)Zr(2)O(12).," Angew. Chem. Int. Ed. Engl., vol. 46, no. 41, 
pp. $7778-81,2007$.

[25] X. Han et al., "Negating interfacial impedance in garnet-based solid-state Li metal batteries," Nat. Mater., vol. 1, no. December, 2016.

[26] M. Ganguli and K. J. Rao, "Studies of ternary Li2SO4-Li2O-P2O5 glasses," $J$. Non. Cryst. Solids, vol. 243, no. 2-3, pp. 251-267, 1999.

[27] M. Rathore and A. Dalvi, "Electrical transport in Li2SO4-Li2O-P2O5 ionic glasses and glass-ceramic composites: A comparative study," Solid State Ionics, vol. 239, pp. 50-55, 2013.

[28] K. U. Jeong, H. D. Chae, C. Il Lim, H. K. Lee, J. H. Ahn, and C. Nah, "Fabrication and characterization of electrolytemembranes based on organoclay/tripropyleneglycol diacrylate/poly(vinylidene fluoride) electrospun nanofiber composites," Polym. Int., vol. 59, no. 2, pp. 249-255, 2010.

[29] A. M. Christie, S. J. Lilley, E. Staunton, and Y. G. Andreev, "Increaing conductivity of crystalline polymer electrolytes nature 2005 ," vol. 433, no. January, pp. 50-53, 2005.

[30] V. Thangadurai and W. Weppner, "Recent progress in solid oxide and lithium ion conducting electrolytes research,” Ionics (Kiel)., vol. 12, no. 1, pp. 81-92, 2006.

[31] G. M. Veith, N. J. Dudney, J. Howe, and J. Nanda, "Spectroscopic characterization of solid discharge products in li-air cells with aprotic carbonate electrolytes," $J$. Phys. Chem. C, vol. 115, no. 29, pp. 14325-14333, 2011.

[32] D. Aurbach, K. Gamolsky, B. Markovsky, Y. Gofer, M. Schmidt, and U. Heider, "On the use of vinylene carbonate (VC) as an additive to electrolyte solutions for Li-ion batteries," Electrochim. Acta, vol. 47, no. 9, pp. 1423-1439, 2002.

[33] S. S. Zhang, "A review on electrolyte additives for lithium-ion batteries," J. Power Sources, vol. 162, no. 2 SPEC. ISS., pp. 1379-1394, 2006.

[34] J. Zhang, X. Huang, H. Wei, J. Fu, Y. Huang, and X. Tang, "Novel PEO-based solid composite polymer electrolytes with inorganic-organic hybrid polyphosphazene microspheres as fillers," J. Appl. Electrochem., vol. 40, no. 8, pp. 1475-1481, 2010.

[35] M. Murayama, N. Sonoyama, A. Yamada, and R. Kanno, "Material design of new lithium ionic conductor, thio-LISICON, in the Li2S-P2S5 system," Solid State Ionics, vol. 170, no. 3-4, pp. 173-180, 2004. 
[36] R. Kanno, T. Hata, Y. Kawamoto, and M. Irie, "Synthesis of a new lithium ionic conductor, thio-LISICON-lithium germanium sulfide system," Solid State Ionics, vol. 130, no. 1, pp. 97-104, 2000.

[37] Y. Du and N. a W. Holzwarth, "Li ion diffusion mechanisms in Li 3 PO 4 electrolytes," vol. 864, p. 427055, 2003.

[38] B. K. Money and K. Hariharan, "Lithium ion conduction in lithium metaphosphate based systems," Appl. Phys. A Mater. Sci. Process., vol. 88, no. 4, pp. 647-652, 2007.

[39] G.-A. Nazri and G. Pistoia, Lithium batteries: science and technology. Springer Science \& Business Media, 2008.

[40] M. Vogel, "Complex lithium ion dynamics in simulated LiPO3 glass studied by means of multitime correlation functions," Phys. Rev. B, vol. 68, no. 18, p. $184301,2003$.

[41] M. Vogel, "Identification of lithium sites in a model of LiPO3 glass: Effects of the local structure and energy landscape on ionic jump dynamics," Phys. Rev. B Condens. Matter Mater. Phys., vol. 70, no. 9, pp. 1-10, 2004.

[42] K. Muruganandam, M. Seshasayee, and S. Patnaik, "An X-ray RDF study of Li2O-P2O5-LiCl glasses,” Solid State Ionics, vol. 89, no. 3, pp. 313-319, 1996.

[43] T. M. Alam, J.-J. Liang, and R. T. Cygan, "Molecular dynamics simulations of the lithium coordination environment in phosphate glasses," Phys. Chem. Chem. Phys., vol. 2, no. 19, pp. 4427-4432, 2000.

[44] C. R. A. Catlow, Computer Modelling in Inorganic Crystallography. Academic Press, San Diego, 1997.

[45] C. R. A. Catlow et al., "Advances in computational studies of energy materials," Philos. Trans. R. Soc. A Math. Phys. Eng. Sci., vol. 368, no. 1923, pp. 3379-3456, 2010.

[46] A. W. Richard Catlow, Alexey Sokol, Computational Approaches to Energy Materials. John Wiley \& Sons, Ltd, 2013.

[47] M. S. Islam et al., "Atomic-scale investigation of defects, dopants, and lithium transport in the LiFePO 4 olivine-type battery material," no. 11, pp. 5085-5092, 2005. 
[48] C. Eames, J. M. Clark, G. Rousse, J. Tarascon, and M. S. Islam, "Lithium migration pathways and van der waals effects in the LiFeSO $4 \mathrm{OH}$ battery material," Chem. Mater., 2014.

[49] T. Maxisch, F. Zhou, and G. Ceder, "Ab initio study of the migration of small polarons in olivine Lix FePO4 and their association with lithium ions and vacancies," Phys. Rev. B - Condens. Matter Mater. Phys., vol. 73, no. 10, pp. 1-6, 2006.

[50] J. M. Clark, S. Nishimura, A. Yamada, and M. S. Islam, "High-voltage pyrophosphate cathode: insights into local structure and lithium-diffusion pathways.," Angew. Chem. Int. Ed. Engl., vol. 51, no. 52, pp. 13149-53, Dec. 2012.

[51] X. Lei, J. Wang, and K. Huang, "Fast li-ion transport in amorphous Li2 Si 2 O 5 : an ab initio molecular dynamics simulation," J. Electrochem. Soc., vol. 163, no. 7, pp. A1401-A1407, 2016.

[52] X. Lei, Y. Jee, and K. Huang, “Amorphous Na 2 Si $2 \mathrm{O} 5$ as a fast $\mathrm{Na}+$ conductor: an ab initio molecular dynamics simulation," J. Mater. Chem. A Mater. energy Sustain., vol. 3, pp. 19920-19927, 2015.

[53] V. Thangadurai, S. Adams, and W. Weppner, "Crystal structure revision and identification of Li+-ion migration pathways in the garnet-like Li5La3M $2 \mathrm{O} 12$ (M = Nb, Ta) oxides," Chem. Mater., vol. 16, no. 16, pp. 2998-3006, 2004.

[54] M. Xu, M. S. Park, J. M. Lee, T. Y. Kim, Y. S. Park, and E. Ma, "Mechanisms of $\mathrm{Li}+$ transport in garnet-type cubic Li 3+xLa 3M $2 \mathrm{O} 12(\mathrm{M}=\mathrm{Te}, \mathrm{Nb}, \mathrm{Zr})$," Phys. Rev. B - Condens. Matter Mater. Phys., vol. 85, no. 5, pp. 1-5, 2012.

[55] W. Kohn and L. J. Sham, "Self-consistent equations including exchange and correlation effects," Phys. Rev., vol. 140, no. 4A, 1965.

[56] M. Orio, D. A. Pantazis, and F. Neese, "Density functional theory," Photosynth. Res., vol. 102, no. 2, pp. 443-453, 2009.

[57] K. Morokuma, "Molecular Orbital Studies of Hydrogen Bonds. III. C $=\mathrm{O} \cdots \mathrm{H}-\mathrm{O}$ Hydrogen Bond in $\mathrm{H}_{2} \mathrm{CO} \cdots \mathrm{H}_{2} \mathrm{O}_{\text {and }} \mathrm{H}_{2} \mathrm{CO} \cdots 2 \mathrm{H}_{2} \mathrm{O}$," J. Chem. Phys., vol. 55, no. 3, pp. 1236-1244, 1971.

[58] M. D. Segall, "Applications of ab initio atomistic simulations to biology," J. Phys. Condens. Matter, vol. 14, no. 11, pp. 2957-2973, 2002. 
[59] D. R. Bowler, T. Miyazaki, and M. J. Gillan, "Recent progress in linear scaling ab initio electronic structure techniques," J. Phys. Condens. Matter, vol. 14, no. 11, pp. 2781-2798, 2002.

[60] J. M. Soler et al., "The SIESTA method for ab initio order-N materials simulation," 2001.

[61] S. Goedecker, "Linear scaling electronic structure methods," Rev. Mod. Phys., vol. 71, no. 4, pp. 1085-1123, 1999.

[62] I. G. Aulstin and N. F. Mott, "Metallic and nonmetallic behavior in transition metal oxides," vol. 168, no. 3927, pp. 71-77, 2016.

[63] W. Metzner and D. Vollhardt, "Correlated lattice fermions in d= dimensions," Phys. Rev. Lett., vol. 62, no. 3, pp. 324-327, 1989.

[64] W. Yang, Y. Zhang, and P. Ayers, "Degenerate ground states and a fractional number of electrons in density and reduced density matrix functional theory," Phys. Rev. Lett., vol. 84, no. 22, pp. 5172-5175, 2000.

[65] V. I. Anisimov, J. Zaanen, and O. K. Andersen, "Band theory and Mott insulators: Hubbard U instead of Stoner I," Phys. Rev. B, vol. 44, no. 3, pp. 943-954, 1991.

[66] P. Giannozzi et al., "QUANTUM ESPRESSO: a modular and open-source software project for quantum simulations of materials.," J. Phys. Condens. Matter, vol. 21, no. 39, p. 395502, 2009.

[67] M. Villarba and H. Jónsson, "Diffusion mechanisms relevant to metal crystal growth: Pt/Pt(111)," Surf. Sci., vol. 317, no. 1-2, pp. 15-36, 1994.

[68] G. Mills and H. Jonsson, "Reversible work transiton state theory: Application to dissociative adsorption of hydrogen," no. 110, 1994.

[69] M. Sørensen, K. Jacobsen, and H. Jónsson, "Thermal diffusion processes in metaltip-surface interactions: contact formation and adatom mobility," Phys. Rev. Lett., vol. 77, no. 25, pp. 5067-5070, 1996.

[70] T. Rasmussen, K. W. Jacobsen, T. Leffers, O. B. Pedersen, S. G. Srinivasan, and H. Jónsson, "Atomistic determination of cross-slip pathway and energetics," Phys. Rev. Lett., vol. 79, no. 19, pp. 3676-3679, 1997.

[71] R. Car and M. Parrinello, "Unified approach for molecular dynamics and densityfunctional theory," Phys. Rev. Lett., vol. 55, no. 22, pp. 2471-2474, 1985. 
[72] T. Atsumi and H. Nakai, "Molecular orbital propagation to accelerate selfconsistent-field convergence in an ab initio molecular dynamics simulation," $J$. Chem. Phys., vol. 128, no. 9, 2008.

[73] C. Zhan et al., "Computational insights into materials and interfaces for capacitive energy storage," Adv. Sci., vol. 4, no. 7, 2017.

[74] JAKES C. PHILLIPS, "Energy-band interpolation scheme based on a pseudopotential," vol. 1210, no. 1952, 1954.

[75] A. Bengtson, H. O. Nam, S. Saha, R. Sakidja, and D. Morgan, "First-principles molecular dynamics modeling of the LiCl-KCl molten salt system," Comput. Mater. Sci., vol. 83, pp. 362-370, 2014.

[76] T. Croteau and G. N. Patey, "Structures and rearrangements of LiCl clusters," $J$. Chem. Phys., vol. 124, no. 24, 2006.

[77] X. Lei, J. Wang, and K. Huang, "Fast li-ion transport in amorphous Li2 Si 2 O 5 : an ab initio molecular dynamics simulation," J. Electrochem. Soc., vol. 163, no. 7, pp. A1401--A1407, 2016.

[78] R. F. W. Bader, "Atoms in molecules," Acc. Chem. Res., vol. 18, no. 1, pp. 9-15, 1985.

[79] Richard F. W. Bader, Atoms in Molecules - A Quantum Theory. Oxford University Press, 1990.

[80] T. T. N.-D. and Y. T. R F W Bader, "Reports on progress in physics charge density of molecules and crystals," Rep. Prog. Phys, 1981.

[81] G. Henkelman, A. Arnaldsson, and H. Jónsson, "A fast and robust algorithm for Bader decomposition of charge density," Comput. Mater. Sci., vol. 36, no. 3, pp. 354-360, 2006.

[82] M. Yu and D. R. Trinkle, "Accurate and efficient algorithm for Bader charge integration," J. Chem. Phys., vol. 134, no. 6, pp. 1-8, 2011.

[83] W. Tang, E. Sanville, and G. Henkelman, "A grid-based Bader analysis algorithm without lattice bias," J. Phys. Condens. Matter, vol. 21, no. 8, p. 84204, 2009.

[84] P. Giannozzi et al., "QUANTUM ESPRESSO: a modular and open-source software project for quantum simulations of materials.," J. Phys. Condens. Matter, vol. 21, no. 39, p. 395502, Sep. 2009. 
[85] G. Kresse and J. Furthmüller, "Efficient iterative schemes for ab initio total-energy calculations using a plane-wave basis set," Phys. Rev. B, vol. 54, no. 16, pp. 11169-11186, 1996.

[86] G. Kresse and J. Hafner, "Ab initio molecular dynamics for liquid metals," Phys. Rev. B, vol. 47, no. 1, pp. 558-561, 1993.

[87] P. E. Blöchl, "Projector augmented-wave method," Phys. Rev. B, vol. 50, no. 24, pp. 17953-17979, 1994.

[88] G. Kresse, "From ultrasoft pseudopotentials to the projector augmented-wave method," Phys. Rev. B, vol. 59, no. 3, pp. 1758-1775, 1999.

[89] J. Perdew, K. Burke, and M. Ernzerhof, “Generalized Gradient Approximation Made Simple.," Phys. Rev. Lett., vol. 77, no. 18, pp. 3865-3868, 1996.

[90] K. Senevirathne, C. S. Day, M. D. Gross, A. Lachgar, and N. A. W. Holzwarth, “A new crystalline LiPON electrolyte: Synthesis, properties, and electronic structure," Solid State Ionics, vol. 233, pp. 95-101, 2013.

[91] N. D. Lepley and N. a. W. Holzwarth, "Computer Modeling of Crystalline Electrolytes: Lithium Thiophosphates and Phosphates," J. Electrochem. Soc., vol. 159, no. 5, p. A538, 2012.

[92] R. W. G. Wyckoff, "Page 65 from the second edition supplement of The Structure of Crystals by Wyckoff R W G. published by Reinhold PublishingCorporation, INC, Camden,N. J. in 1935 \& http://database.iem.ac.ru/mincryst/," Second Ed. Suppl. Struct. Cryst., pp. 65-65, 1935.

[93] J. Zemann, "Die Kristallstruktur von Lithiumphosphat, Li3PO4," Acta Crystallogr., vol. 13, no. 11, pp. 863-867, Nov. 1960.

[94] O. V. Yakubovich and V. $\sim$ S. Urusov, "Electron density distribution in lithiophosphatite Li\$_\{3\}\$PO\$_\{4\}\$; crystallochemical features of orthophosphates with hexagonal close packing," Crystallogr. Reports, vol. 42, pp. 261-268, Mar. 1997.

[95] G. Hester, T. Heitmann, M. Tyagi, M. Rathore, A. Dalvi, and S. Mitra, "Neutron scattering studies of lithium-ion diffusion in ternary phosphate glasses," MRS Adv., vol. 1, no. 45, pp. 3057-3062, 2016.

[96] J. C. Guitel, "Structure cristalline de polyphosphate de lithium LiPO3," Acta Crystallogr. Sect. B Struct., pp. 2960-2966, 1976. 
[97] J. Guitel, "Structure cristalline de polyphosphate de lithium LiPO3," Acta Crystallogr. Sect. B Struct., pp. 2960-2966, 1976.

[98] G. Henkelman, B. P. Uberuaga, H. Jónsson, and G. Henkelman, “A climbing image nudged elastic band method for finding saddle points and minimum energy paths A climbing image nudged elastic band method for finding saddle points and minimum energy paths," vol. 9901, no. 2000, pp. 1-5, 2011.

[99] S. Adams, "Lithium ion pathways in LiFePO 4 and related olivines," J. Solid State Electrochem., vol. 14, no. 10, pp. 1787-1792, 2010.

[100] M. Wenger, T. Armbruster, U. Bern, and C.- Bern, "Crystal chemistry of lithium : oxygen coordination and bonding," Eur. J. Miner., vol. 3, pp. 387-399, 1991.

[101] J. Smekens et al., "Influence of electrode density on the performance of Li-ion batteries: Experimental and simulation results," Energies, vol. 9, no. 2, pp. 1-12, 2016.

[102] Y. Zhu, X. He, and Y. Mo, "First principles study on electrochemical and chemical stability of solid electrolyte-electrode interfaces in all-solid-state Li-ion batteries," J. Mater. Chem. A, vol. 4, no. 9, pp. 3253-3266, 2016.

[103] Y. Zhu, X. He, and Y. Mo, "Strategies based on nitride materials chemistry to stabilize Li metal anode," Adv. Sci., vol. 4, no. 8, pp. 1-11, 2017.

[104] B. K. Money and K. Hariharan, "Lithium ion conduction in lithium metaphosphate based systems," Appl. Phys. A Mater. Sci. Process., vol. 88, no. 4, pp. 647-652, 2007.

[105] J. L. Souquet, M. Duclot, and M. Levy, "Ionic transport mechanisms in oxide based glasses in the supercooled and glassy states," Solid state ionics, vol. 105, no. 1-4, pp. 237-242, 1998.

[106] T. Kitagawa, K. Yabuki, and R. . Young, "An investigation into the relationship between processing, structure and properties for high-modulus PBO fibres. Part 1. Raman band shifts and broadening in tension and compression," Polymer (Guildf)., vol. 42, no. 5, pp. 2101-2112, 2001.

[107] D. Tuschel, "Why are the raman spectra of crystalline and amorphous solids different?," Spectroscopy, vol. 32, no. 3, pp. 26-33, 2017. 


\section{APPENDIX}

Code used in QUANTUM ESPRESSO suite:

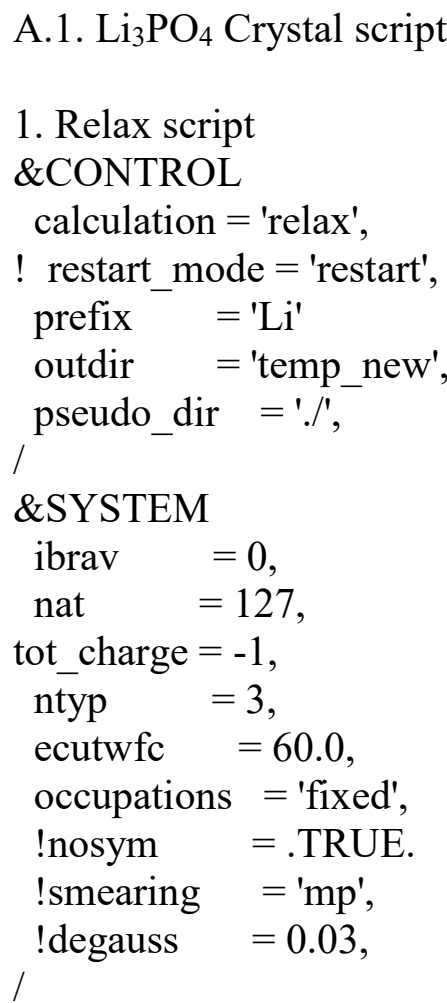

\section{\&ELECTRONS}

electron_maxstep $=300$, mixing_mode $=$ 'plain', mixing_beta $=0.7$, mixing_ndim $=12$, diagonalization $=$ 'cg', I

\&ions

ion_dynamics = 'bfgs', I

\section{\&CELL} cell_dynamics $=$ 'bfgs' , press $=0$, cell_factor $=3$, /

\section{ATOMIC_SPECIES}

Li 6.941 Li.pbe-s-van_ak.UPF

O 15.9994 O.pbe-van_ak.UPF

P 30.973762 P.pbe-van_ak.UPF 


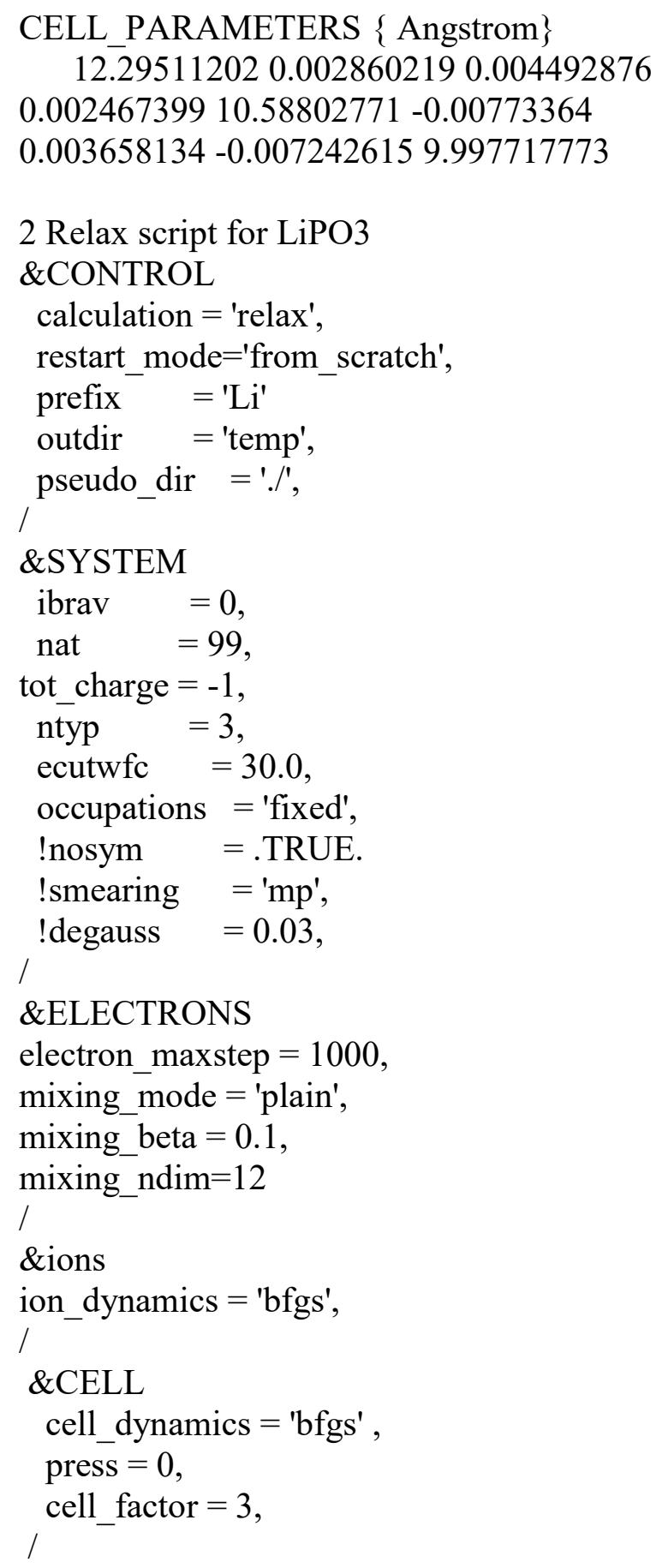

ATOMIC_SPECIES

Li 6.941 Li.pbe-s-van ak.UPF

O 15.9994 O.pbe-van_ak.UPF

P 30.973762 P.pbe-van_ak.UPF

CELL_PARAMETERS \{ Angstrom

$\begin{array}{lll}17.0336723328 & 0.0000000000 & 0.0000000000\end{array}$

$\begin{array}{lll}0.0017676182 & 5.5635315948 & 0.0000000000\end{array}$

$\begin{array}{lll}-2.3835290088 & -0.0315687276 & 13.2267909119\end{array}$ 
1. Crystal volume relax:

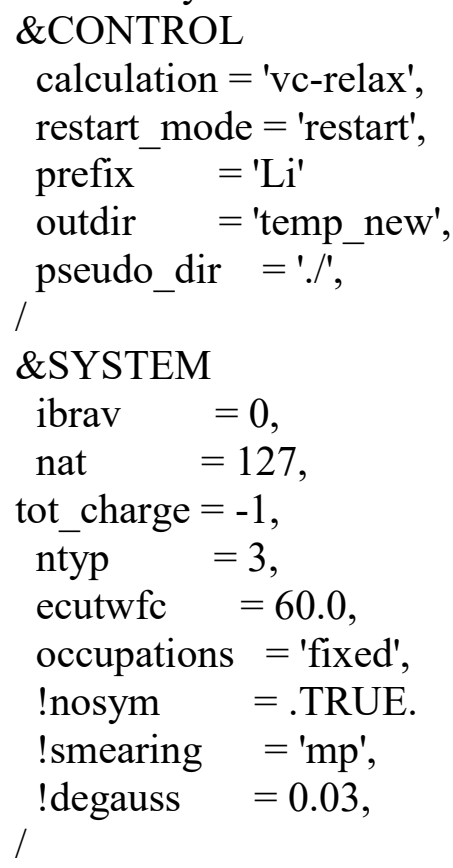

3. NEB script:

BEGIN

BEGIN_PATH_INPUT 


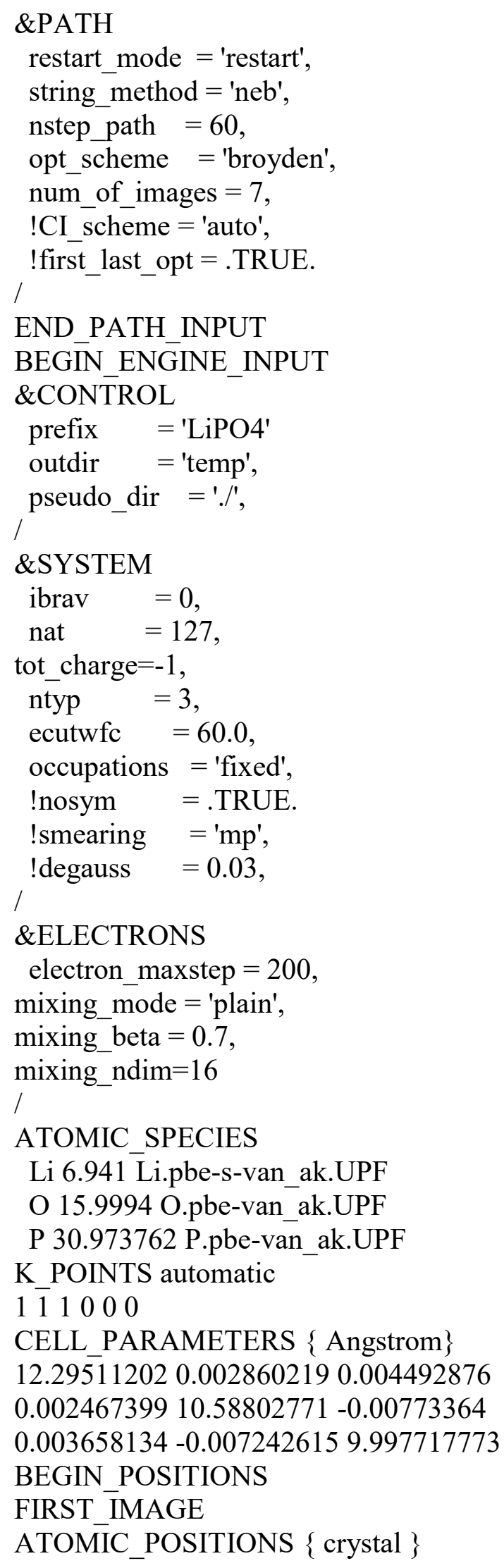




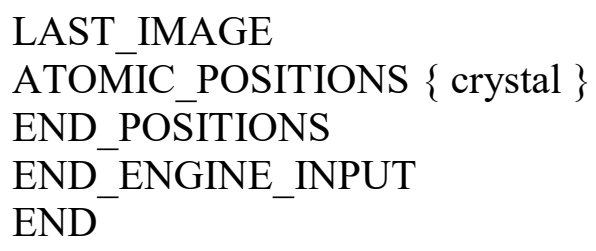

4. Self-consistancy for charge calculation(VASP):

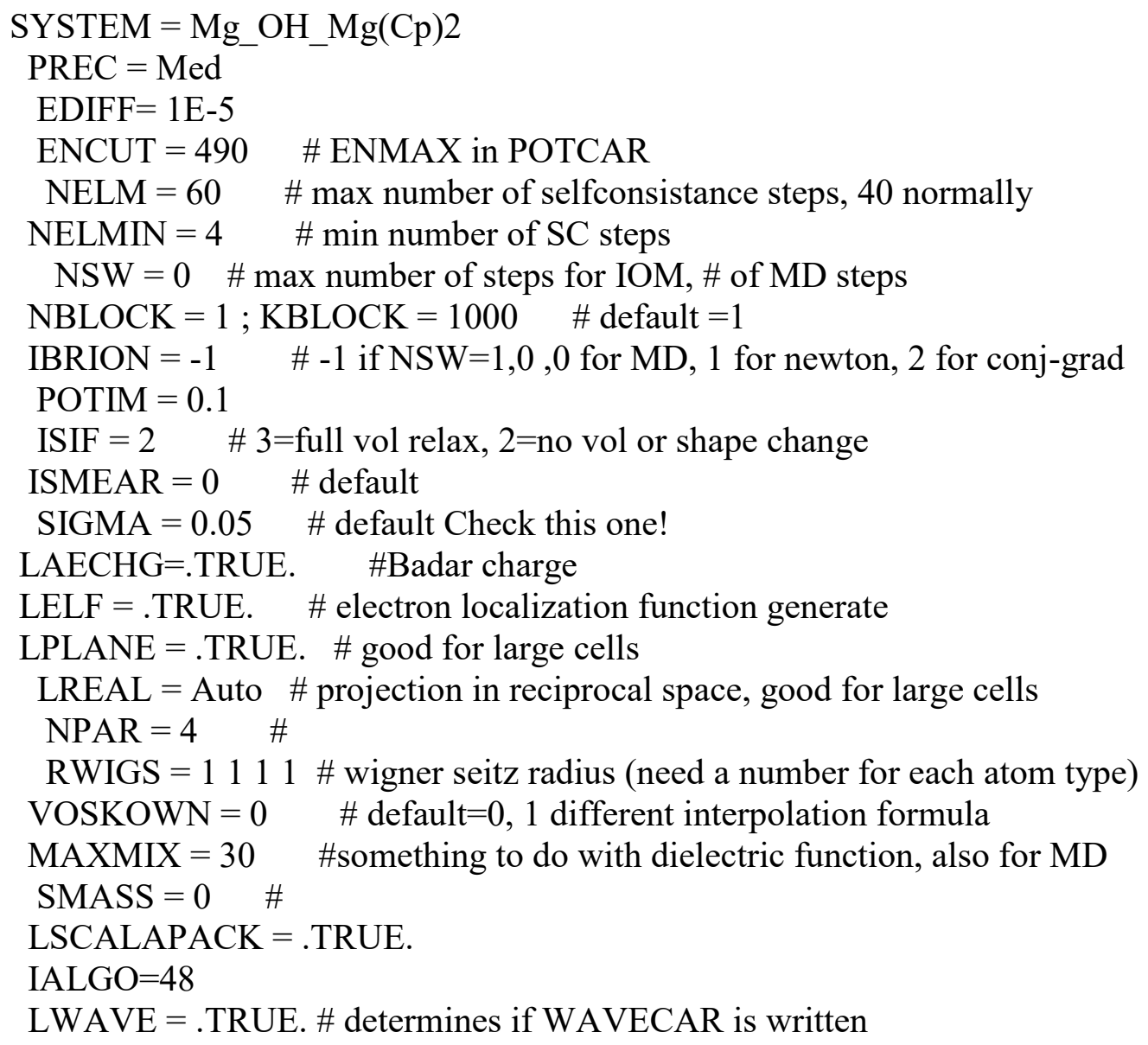

5. Bader charges analysis script:

For charge calculation Bader charge analysis has been used. The charge information is already can be found in charge density (CHGCAR) file in VASP code. However the file contains only the valance charge density information. To implement Bader analysis, it is important to calculate the core region charge as well. Bader analysis assumes the core has the most charge. In some pseudopotential, the core charge is replaced by minimizing 
calculation cost. Recently VASP introduced a module (aedens) which write the core charge of the atomic centers to the charge density file. For that one flag:

LAECHG=.TRUE need to be in the INCAR file. The core charge then is written o the AECCAR0 and the valance charge are written to AECCAR2 files. These files can be summed together using chgsum.pl script using bellow command.

chgsum.pl AECCAR0 AECCAR2

This command writes the total charge to CHGCAR_sum file which can be used by the bader code to generate the cahrge for each atom in the structure using the command:

bader CHGCAR -ref CHGCAR_sum

One important thing to notice is to use the proper $\mathrm{NG}(\mathrm{X}, \mathrm{Y}, \mathrm{Z})$. To do so, I have run $\mathrm{SCF}$ several time to adopt correct $\mathrm{NG}(\mathrm{X}, \mathrm{YZ})$ for the system. Also a fine $\mathrm{fft}$ grid is used.

A.2 Amorphous analysis scripts:

1. NVT script to cooldown temperature:

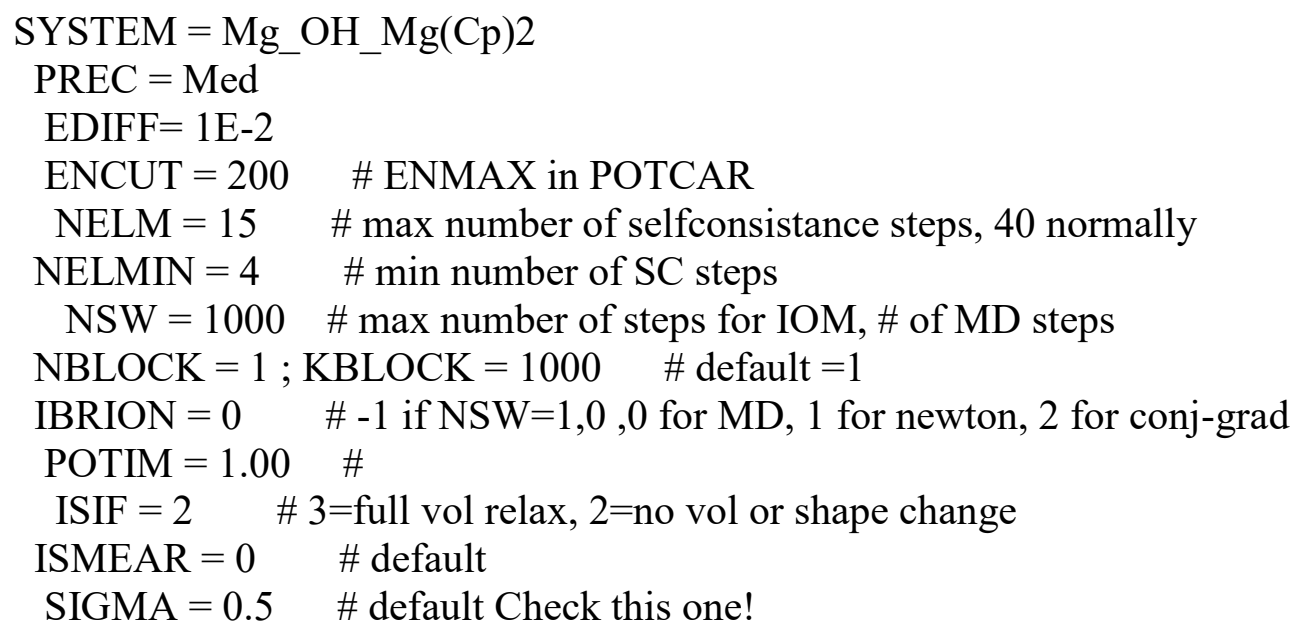


LPLANE $=$.TRUE. $\quad \#$ good for large cells

LREAL $=$.TRUE. \# projection in reciprocal space, good for large cells

$\mathrm{NPAR}=4 \quad \#$

RWIGS = 1111 \# wigner seitz radius (need a number for each atom type)

$\mathrm{VOSKOWN}=0 \quad$ \# default=0, 1 different interpolation formula

ALGO $=$ VeryFast \#

MAXMIX $=30 \quad$ \#something to do with dielectric function, also for MD

ISYM $=0 \quad$ \#switch of symmetry, for MD

SMASS $=0 \quad \#$

TEBEG $=3000 \quad$ \#temperature for MD

TEEND $=2500$

LSCALAPACK $=$.TRUE.

$\mathrm{IALGO}=48$

LWAVE $=$.TRUE. $\#$ determines if WAVECAR is written

2. NPT script to equilibrate pressure:

Prec $=$ Med

LPLANE $=$.TRUE. \# good for large cells

$\mathrm{LREAL}=$.TRUE. \# projection in reciprocal space, good for large cells

RWIGS = $\begin{array}{lllll}1 & 1 & 1\end{array}$ \# wigner seitz radius (need a number for each atom type)

LWAVE = .FALSE. \# determines if WAVECAR is written

$\mathrm{LCHARG}=. \mathrm{FALSE}$. \# determines if CHGCAR/CHG are written

MAXMIX $=30 \quad$ \#something to do with dielectric function, also for MD

ISYM $=0 \quad$ \#switch of symmetry, for MD

$\mathrm{SMASS}=0 \quad \#$

LSCALAPACK $=$.TRUE.

ALGO $=$ VeryFast

$\mathrm{IALGO}=48$

$\mathrm{NSW}=2000$

$\operatorname{ISMEAR}=-1 ; \mathrm{SIGMA}=0.2 \quad$ \# smearing method

$\mathrm{ALGO}=\mathrm{Med}$

$\mathrm{EDIFF}=1 \mathrm{E}-3 ; \mathrm{NELMIN}=5 \quad$ \#

$\mathrm{LREAL}=$ Auto \# real space projector

MAXMIX $=40 ;$ AMIX $=1.0 ;$ BMIX $=1.0 \#$ optimize charge mixer for $\mathrm{Si}$

$\mathrm{LCHARG}=$ False

$\mathrm{IALGO}=48$

$\mathrm{NELMIN}=5$

ISYM $=0$

$\mathrm{NPAR}=8$

ADDGRID $=$ TRUE

$\mathrm{ENCUT}=440$

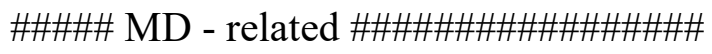

RANDOM_SEED $=\quad 689642361 \quad 62398$

$\mathrm{IBRION}=\overline{0} \quad \# \mathrm{MD}$

$\mathrm{ISIF}=3 \quad$ \# variable lattice parameters

POTIM=2 


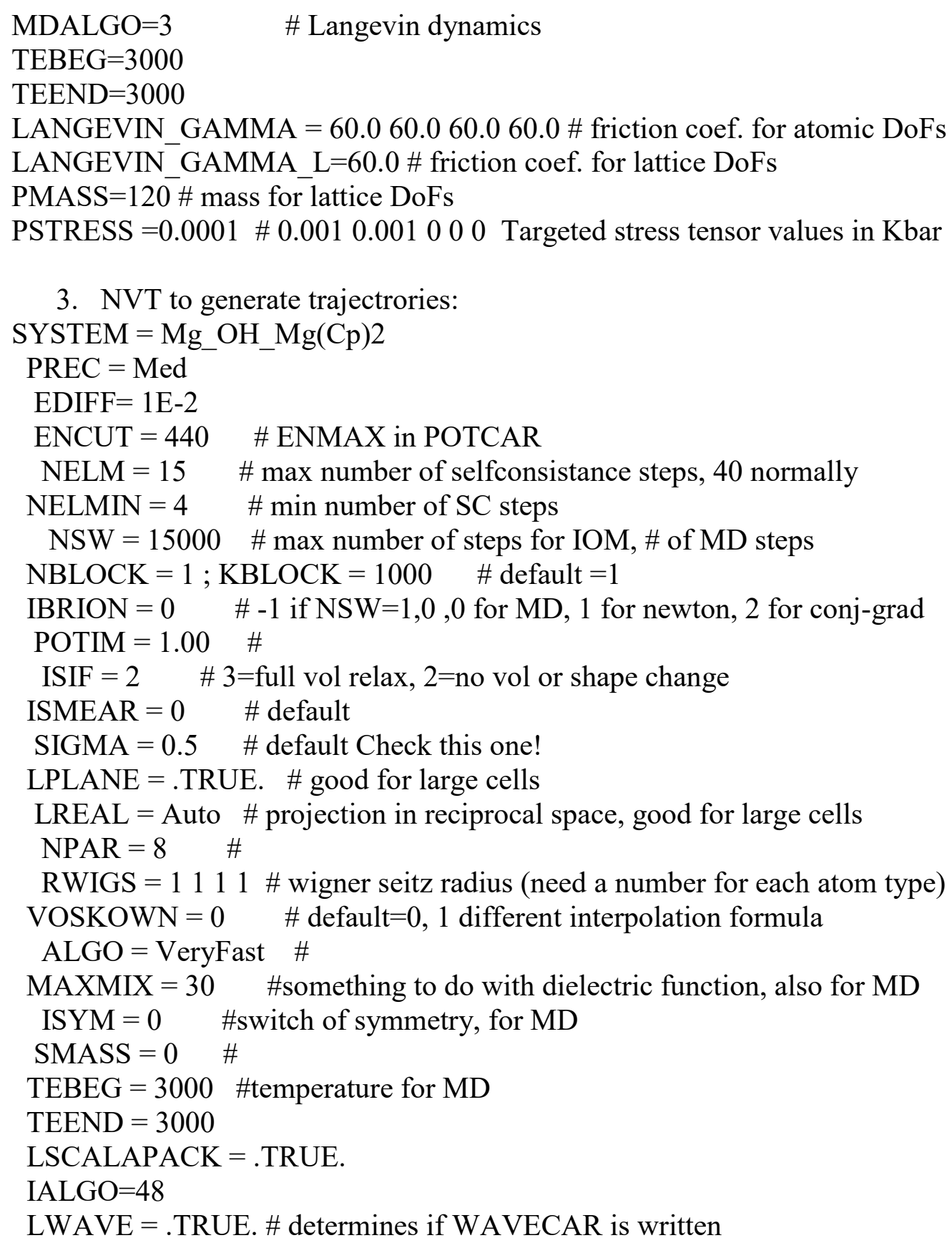

